\title{
Comprehensive clinically oriented workflow for nucleotide level resolution and interpretation in prenatal diagnosis of de novo apparently balanced chromosomal translocations in their genomic landscape
}

DOI:

10.1007/s00439-020-02121-x

\section{Document Version}

Accepted author manuscript

Link to publication record in Manchester Research Explorer

\section{Citation for published version (APA):}

David, D., Freixo, J. P., Fino, J., Carvalho, I., Marques, M., Cardoso, M., Piña-aguilar, R. E., \& Morton, C. C. (2020). Comprehensive clinically oriented workflow for nucleotide level resolution and interpretation in prenatal diagnosis of de novo apparently balanced chromosomal translocations in their genomic landscape. Human Genetics. https://doi.org/10.1007/s00439-020-02121-x

\section{Published in:}

Human Genetics

\section{Citing this paper}

Please note that where the full-text provided on Manchester Research Explorer is the Author Accepted Manuscript or Proof version this may differ from the final Published version. If citing, it is advised that you check and use the publisher's definitive version.

\section{General rights}

Copyright and moral rights for the publications made accessible in the Research Explorer are retained by the authors and/or other copyright owners and it is a condition of accessing publications that users recognise and abide by the legal requirements associated with these rights.

\section{Takedown policy}

If you believe that this document breaches copyright please refer to the University of Manchester's Takedown Procedures [http://man.ac.uk/04Y6Bo] or contact uml.scholarlycommunications@manchester.ac.uk providing relevant details, so we can investigate your claim.

\section{OPEN ACCESS}


Dezső David $^{1}$ João Freixo $^{2 *}$ Joana Fino $^{1 *}$ Inês Carvalho ${ }^{2}$ Mariana Marques $^{1}$ Manuela $^{2}$ $\operatorname{Cardoso}^{1}$ Raul E. Piña-Aguilar ${ }^{3,4}$ Cynthia C. Morton ${ }^{3-7}$

Comprehensive clinically oriented workflow for nucleotide level resolution and interpretation in prenatal diagnosis of de novo apparently balanced chromosomal translocations in their genomic landscape

${ }^{1}$ National Health Institute Doutor Ricardo Jorge, Department of Human Genetics, Lisbon, Portugal;

${ }^{2}$ Central Lisbon Hospital Center (CHLC), Department of Medical Genetics, Lisbon, Portugal;

${ }^{3}$ Harvard Medical School, Boston, MA, United States;

${ }^{4}$ Departments of Obstetrics and Gynecology and of ${ }^{5}$ Pathology, Brigham and Women's Hospital, Boston, MA, United States;

${ }^{6}$ Broad Institute of MIT and Harvard, Cambridge, MA, United States;

${ }^{7}$ University of Manchester, Manchester Academic Health Science Center, Manchester, UK

*These authors contributed equally to the work

\section{Corresponding author:}

Dezső David, Ph.D.

Department of Human Genetics

National Health Institute Doutor Ricardo Jorge

Av. Padre Cruz, 1649-016 Lisbon, Portugal

email: dezso.david@insa.min-saude.pt

Fax: (+351) 217526410

Telephone: (+351) 217519322 
Acknowledgments We dedicate this article to Maria Guida Boavida who founded the Human Genetics Laboratory as the precursor of the current Department of Human Genetics at the National Health Institute Doutor Ricardo Jorge, Lisbon, Portugal. She and her colleagues performed the first chromosomal prenatal diagnosis from cultured amniocytes in Portugal in July 1977 (no. 77180). However, the first translocation in PND, a paternally inherited Robertsonian t(13;14) (no. 80321), was only identified in June 1980. Almost 40 years later, we report application of an liGS-based approach for identification of dnBCAs breakpoints in PND.

First, we are grateful to the family members for their involvement in this study. We thank Dr. Michael Talkowski and members of his lab for library preparation and sequencing data generation. We thank Jader Cruz for echographic diagnosis of fetal heart defects, to Hildeberto Correia, Ana Cristina Alves and Bárbara Marques for conventional prenatal diagnosis, and to Márcia Rodrigues, Sofia Nunes, Teresa Kay, and Rui Gonçalves for their contribution to the project. C.C.M. is supported (GM061354) by the National Institutes of Health (USA) and the NIHR Manchester Biomedical Research Centre, UK. This research was supported by national funds through FCT - Fundação para a Ciência e a Tecnologia, Research Grant HMSP-ICT/0016/2013 of the Harvard Medical School - Portugal Program in Translational Research and Information. 


\begin{abstract}
We present a comprehensive clinically oriented workflow for large-insert genome sequencing (liGS)-based nucleotide level resolution and interpretation of de novo ( $\mathrm{dn}$ ) apparently balanced chromosomal abnormalities (BCA) in prenatal diagnosis (PND). Retrospective or concomitant with conventional PND and liGS, molecular and newly developed clinically inspired bioinformatic tools (TAD-GConTool and CNV-ConTool) are applied to analyze and assess the functional and phenotypic outcome of dn structural variants (dnSVs). Retrospective analysis of four phenotype-associated dnSVs identified during conventional PND precisely reveal the genomic elements disrupted by the translocation breakpoints. Identification of autosomal dominant disease due to disruption of ANKS1B and WDR26 by $\mathrm{t}(12 ; 17)(\mathrm{q} 23.1 ; \mathrm{q} 21.33) \mathrm{dn}$ and $\mathrm{t}(1 ; 3)(\mathrm{q} 24.11 ; \mathrm{p} 25.3) \mathrm{dn}$ breakpoints, respectively, substantiated the proposed workflow. We then applied this workflow to two ongoing prenatal cases with apparently balanced dnBCAs: 46,XX,t(16;17)(q24;q21.3)dn referred for increased risk on combined first trimester screening and 46,XY,t(2;19)(p13;q13.1)dn referred due to a previous trisomy 21 pregnancy. Translocation breakpoints in the $\mathrm{t}(16 ; 17)$ involve ANKRD11 and WNT3 and disruption of ANKRD11 resulted in KBG syndrome confirmed in postnatal follow-up. Breakpoints in the $\mathrm{t}(2 ; 19)$ are within ATP6VIB1 and the 3' UTR of CEP89, and are not interpreted to cause disease. Genotype-phenotype correlation confirms the causative role of WDR26 in the Skraban-Deardorff and 1q41q42 microdeletion phenocopy syndromes, and that disruption of $A N K S 1 B$ causes $A N K S 1 B$ haploinsufficiency syndrome. In sum, we show that an liGS-based approach can be realized in PND care providing additional information concerning clinical outcomes of dnBCAs in patients with such rearrangements.
\end{abstract}

Keywords: Balanced chromosomal abnormality (BCA); PND care; liGS; KBG syndrome; ANKS1B haploinsufficiency syndrome; Skraban-Deardorff syndrome; TAD-GConTool; CNV-ConTool 


\section{Introduction}

A causal relationship between a balanced chromosomal abnormality (BCA) and a congenital anomaly is predicted in up to $40 \%$ of cases presenting a clinical phenotype associated BCA (Redin et al. 2017). Recognition of de novo (dn) BCAs leading to disorders constitutes a formidable challenge in prenatal diagnosis (PND). Conventional low-resolution karyotyping remains the standard approach for assigning rearrangement breakpoints of cytogenetically visible dnBCAs in the prenatal setting. Breakpoints of some BCAs have been localized though molecular cytogenomic approaches including FISH, but high-resolution chromosomal microarrays (CMA) are generally insensitive to BCAs (David et al. 2003). More recently, massively parallel sequencing-based methods have been used facilitating nucleotide level resolution of BCAs (Chen et al. 2008; Talkowski et al. 2011).

Long-insert genome sequencing (liGS), with high physical coverage and low sequence depth, has been applied within an actionable timeframe of a PND, for precise identification of BCA breakpoints (Talkowski et al. 2012; Ordulu et al. 2016). BCAs must also be evaluated in the context of copy-number variation $(\mathrm{CNV})$ burden, and the relevance and expanding knowledge of topologically associated domains (TADs) in mechanisms of disease (Dixon et al. 2012; Lupiáñez et al. 2015).

In the present study, we apply the liGS approach for identification of structural variant (SV) breakpoints in four retrospectively analyzed dnBCAs identified during conventional PND and in two ongoing PNDs with dnBCAs. Two bioinformatic tools to assist prediction of the phenotypic outcome of SVs and CNVs in the routine clinical setting were developed including evaluation of the local genomic landscape in which these dnBCAs occurred. Finally, we consider the predictability of the phenotypic outcome of these dnBCAs identified during PND. 


\section{Materials and methods}

\section{Patients, karyotyping and CMA}

Two fetal and four adolescent probands with dnBCAs identified by fetal karyotyping during a conventional PND protocol and their family members were analyzed. Karyotyping and CMA are described in Supplementary Material and Methods.

\section{liGS library preparation, sequencing, bioinformatic analysis and resolution}

liGS library preparation, sequencing, and bioinformatic analysis of sequencing data were carried out essentially as described by Talkowski et al. (2011) and Collins et al. (2017). Briefly, after aligning read-pairs against the reference genome, chimeric and improper readpairs were selected, categorized, clustered and filtered against a so-called blacklist, a list of genomic regions with systematic short-read mappability biases, with an overlap cut-off $\geq$ 30\% (Collins et al. 2017). Based on cluster analysis, different types of balanced and unbalanced SVs such as translocations, insertions (ins), inversions (inv), complex (cx) SV, deletions (del) and tandem duplications (dup) can be identified by liGS. The resolution of liGS is equivalent to the median insert size plus twice the S.D., i.e., $\sim 4.5 \mathrm{~kb}$. SVs identified in 689 participants with autism spectrum disorder were used as an SV reference dataset (SVref dataset; Collins et al. 2017). Deletions and tandem duplications identified by depthof-coverage and improper cluster analysis were cross validated (Klambauer et al. 2012; Collins et al. 2016) and analyzed using our CNV-ConTool.

As long as read-pair clusters do not overlap low-complexity regions, our clinically oriented pipeline includes all translocations, ins, del and dup above $30 \mathrm{~kb}$, and cx SV above $10 \mathrm{~kb}$. A more detailed description is available in Supplementary Material.

\section{Identification of cluster-specific split-reads, CNVs and bioinformatic tools}

For identification of cluster-specific split-reads encompassing BCA or SV breakpoints, a custom Python algorithm was developed and applied. This process uses read-pairs with one of the reads mapped within a breakpoint cluster and the respective paired read unmapped. Detailed description of this algorithm is available in Supplementary Material. 
To assist prediction of the phenotypic outcome of SV and CNVs, two bioinformatic tools were developed. TAD-Gene Content Tool (TAD-GConTool) using TAD data from Dixon et al. (2012) and Moore et al. (2015), identifies breakpoint spanning and flanking TADs and retrieves a series of protein-coding and non-coding RNA genes and genomic elements localized within the TADs, as well as associated structural and functional information. Additionally, this tool has the ability to construct the sequence-based nomenclature of the SVs according to the International System for Human Cytogenomic Nomenclature (ISCN) 2016. This tool will be updated in concert with revisions to ISCN 2016, anticipated to be ISCN 2020. CNV-Content Tool (CNV-ConTool) was developed to search for overlap between patient-specific CNVs and those from public databases. This second tool also retrieves data on genes affected by these CNVs. Detailed descriptions of both bioinformatic tools are available in Supplementary Material and Methods.

Both TAD-GConTool and CNV-ConTool can be accessed online at www.dgrctoolsinsa.min-saude.pt. Source codes are available at https://github.com/DGRC-PT/.

\section{Amplification of junction fragments}

Amplification conditions for junction and control fragments of BCA and proband-specific CNVs are summarized in Supplementary Table 1.

\section{Lymphoblastoid cell lines (LCLs), RNA extractions and expression studies}

Establishment of LCLs from peripheral blood lymphocytes, extraction of RNAs from LCLs, peripheral blood and amniocytes, and quality assessment and quantification of RNAs were performed essentially as described previously (David et al. 2003).

Genome-wide assessment of gene expression levels in LCL or amniocytes of the probands and controls were performed using the Affymetrix Human Transcriptome Array 2.0 (HTA 2.0, ThermoFisher Scientific). Sample and array processing and data analysis were carried out according to the manufacturer's instructions and are detailed in the Supplementary Material and Methods.

\section{Variant interpretation and disease prediction}


Variants produced by liGS were interpreted according to ACMG sequence based variant criteria (Richards et al. 2015) or CNV criteria (Riggs et al. 2019). For disease prediction (Table 1) bespoke criteria were developed to guide clinicians in the interpretation of sequencing results and ACMG variant classification, as follows:

Disease causing - a structural variant resulting in loss-of-function (LoF) of an annotated gene transcript causing an autosomal dominant $(\mathrm{AD})$ clinically relevant or major developmental disorder, where LoF is a known mechanism of the disease;

Low potential of disease - a structural variant resulting in LoF of an annotated gene transcript solely causing autosomal recessive (AR) disorders or not associated with an AD clinically relevant or major developmental disorder causing gene localized within the breakpoint topological associating domains (bpTADs);

Non-disease causing - a structural variant resulting in no disruption of protein-coding genes within the bpTADs, no human pathology reported to be associated with genomic elements localized within the bpTADs or no statistically significant GWAS data and/or data supporting at least partial overlap between the genetic traits associated with the affected genomic region and the patient phenotype; and

Disease plausible - a structural variant resulting in disruption of an annotated gene transcript intolerant to LoF variants but not yet associated with human disorders, affected gene reported with an important biological function, or convergent genomic and biological evidence (GWAS, gene expression, phenotypic data and other) supporting at least partial involvement of the disrupted gene in the patient clinical phenotype. 


\section{Results}

\section{Patient medical histories}

A 39 year-old female presented with an elevated risk for aneuploidy following $1^{\text {st }}$ trimester combined tests with increased nuchal translucency $\left(4.1 \mathrm{~mm},>95^{\text {th }}\right.$ percentile $)$. Chorionic villus sampling (CVS) was performed at 14 weeks gestation for karyotyping of the fetus (designated DGRC0016). Neither parent had any relevant family medical history.

Ultrasound examination at 20 weeks revealed hypoplastic nasal bone and atrioventricular septal defect (AVSD) with ventricular septal defect (VSD) confirmed by fetal echocardiography. Besides AVSD and fetal growth restriction $\left(5^{\text {th }}\right.$ centile $)$, no other fetal anomalies were observed on subsequent evaluations.

Postnatal echocardiogram confirmed the reported AVSD with small VSD but without hemodynamic compromise. At 20 months of age, DGCR0016 presented with developmental delay, most evident in the postural control and locomotor areas, growth restriction and the characteristic facial gestalt to fulfill the criteria for a clinical diagnosis of KBG syndrome (KBGS) (Supplementary Table 2) (Low et al. 2016).

The 40 year-old mother of the second prenatal proband, DGCR0019, had a history of previous pregnancy termination due to trisomy 21 and was referred for amniocentesis at 17 weeks of gestation due to maternal anxiety. Postnatal medical examination of the newborn was phenotypically normal. Besides slightly hypohidrotic skin noticed at four months of age, no other health problem was noted.

Clinical phenotypes of retrospectively analyzed probands are described in Supplementary Results (DGRC0006 - t(8;14), DGRC0013 - inv(13), DGRC0025 - t(12;17), and DGRC0030 - $\mathrm{t}(1 ; 3))$ and summarized in Table 1. Clinical features of probands DGRC0006 and DGRC0013 do not match a specific genetic diagnosis, whereas DGRC0025 (Supplementary Fig. 1 and Supplementary Table 3) and DGRC0030 (Supplementary Fig. 2 and Supplementary Table 4) present clinical phenotypes matching a recently reported ANKSIB haploinsufficiency syndrome (Carbonell et al. 2019) and Skraban-Deardorff syndrome (SKDEAS OMIM \#617616) (Skraban et al. 2017), respectively. 


\section{Conventional prenatal diagnosis}

Cytogenetic analysis of the CVS of DGRC0016 revealed a de novo apparently balanced reciprocal translocation, 46,XX,t(16;17)(q24;q21.3)dn (Fig. 1a, b). CMA identified an 810 $\mathrm{kb}$ de novo deletion at $8 \mathrm{q} 24.21$ interpreted to be a variant of uncertain significance based on a total score of $-0.15(1 \mathrm{~A}, 2 \mathrm{H}, 3 \mathrm{~A}, 4 \mathrm{I}$ and $5 \mathrm{~A}$ criteria) obtained from the ACMG CNV interpretation guidelines (Riggs et al. 2019). Karyotyping of the amniotic fluid cells of DGRC0019 revealed a de novo apparently balanced reciprocal translocation, 46,XY,t(2;19)(p13;q13.1)dn (Fig. 2a, b).The balanced nature of the translocations was confirmed by CMA and breakpoints mapped on average with a $7 \mathrm{Mb}$ resolution by karyotyping.

\section{Detection of SVs from liGS data}

Two prenatal and four retrospective probands were sequenced using Illumina short-read (25 bp) sequencing of liGS libraries. Metrics for the libraries are summarized in Supplementary Table 5. Physical coverage was between 42 to 88 -fold whereas sequence depth was about one-fold. Chimeric and improper read-pairs ranged between approximately 4 to $8 \%$.

SVs were identified at liGS resolution of $\sim 4.5 \mathrm{~kb}$, but clinical reported at resolution of $\geq$ $30 \mathrm{~kb}$. A summary of identified chimeric read-pair clusters denoting translocations, ins, inv and cx SV are shown in Supplementary Table 6. At clinical resolution, on average three fully resolved, novel or non-polymorphic $(<1 \%)$ SVs were discovered, whereas at liGS resolution, an average of five additional novel or non-polymorphic SVs were identified.

Likewise, a summary of identified del and dup is shown in Supplementary Table 7. At a clinical resolution of $\geq 30 \mathrm{~kb}$, an average of $18 \mathrm{del}$ and dup were identified per proband, but only four del and six dup are considered novel or non-polymorphic $(<1 \%)$ based on the SVref dataset (Collins et al. 2017). At liGS resolution, after filtering, an additional 14 del and 15 dup were detected per patient.

\section{Identification of breakpoints at nucleotide resolution}

liGS of the fetal DNA sample DGRC0016 identified the 16q breakpoint within a $70 \mathrm{bp}$ region (chr16:89,401,663-89,401,732) at 16q24.3, and the 17q breakpoint was delimited to a 
2,300 bp region (chr17:46,781,986-46,784,286) at 17q21.31 (Fig. 1). A split-read was found at the der(17) breakpoint. Sequencing of the second fetal DNA sample DGRC0019 identified the $2 p$ breakpoint within a $485 \mathrm{bp}$ fragment $(\mathrm{chr} 2: 70,941,289-70,941,773)$ at $2 \mathrm{p} 13.3$, and the 19q breakpoint was mapped within a 132 bp sequence (chr19:32,878,469-32,878,600) at 19q13.11. Junction fragments for both cases were amplified and Sanger sequenced (Supplementary Table 1 and Supplementary Figs. 3 and 4).

The karyotype of DGRC0016 was revised to $\mathrm{t}(16 ; 17)(16 \mathrm{pter} \rightarrow 16 \mathrm{q} 24.3:: 17 \mathrm{q} 21.31 \rightarrow 17 \mathrm{qter} ; 17 \mathrm{pter} \rightarrow 17 \mathrm{q} 21.31:: 16 \mathrm{q} 24.3 \rightarrow 16 \mathrm{qter}) \mathrm{dn}$, and according to next-gen cytogenetics nomenclature (Ordulu et al. 2014) is described as 46,XX,t(16;17)(q24;q21.3)dn.seq[GRCh38] $\mathrm{t}(16 ; 17)(16 \mathrm{pter} \rightarrow 16 \mathrm{q} 24.3(89,401,715):: 17 \mathrm{q} 21.31(46,784,035) \rightarrow 17 \mathrm{qter} ; 17 \mathrm{pter} \rightarrow 17 \mathrm{p} 21.31(4$ $6,781,998:: 16 \mathrm{q} 24.3(89,401,718) \rightarrow 16 \mathrm{qter}) \mathrm{dn}$. The translocation was reclassified as unbalanced due to the 2,036 bp deletion identified at the 17q21.31 breakpoint (Supplementary Fig. 3).

The karyotype of DGRC0019 was revised to $\mathrm{t}(2 ; 19)(19 \mathrm{qter} \rightarrow 19 \mathrm{q} 13.11:: 2 \mathrm{p} 13.3 \rightarrow 2 \mathrm{qter}$; 19pter->19q13.11::2p13.3 $\rightarrow 2$ pter)dn, and according to next-gen cytogenetics nomenclature is described as 46,XY,t(2;19)(p13;q13.1)dn.seq[GRCh38] t(2;19)(19qter()$\rightarrow 19 \mathrm{q} 13.11(32,878,515):: 2 \mathrm{p} 13.3(+)(70,941,507) \rightarrow 2 \mathrm{qter} ; 19 \mathrm{pter} \rightarrow 19 \mathrm{q} 13.11(+)(32,878,512):$ $:$ CATA::2p13.3(-)(70,941,502) $\rightarrow 2$ pter $)$ dn.

\section{Characterization of breakpoint regions}

In DGRC0016, the 16q24.3 breakpoint at position chr16:89,401,715 disrupts IVS3 of ANKRD11 (Ankrd11 repeat domain 11, OMIM *611192), whereas the 17q21.31 breakpoint at position chr17:46,781,998 disrupts IVS1 of WNT3 (Wnt family member 3, OMIM *165330) (Fig. 1). Haploinsufficiency of ANKRD11 causes AD KBGS ( (Sirmaci et al. 2011).

Homozygous pathogenic variants in WNT3 are associated (but not yet independently confirmed) with recessive tetra-amelia syndrome-1 (TETAMS1, OMIM \#165330), a severe malformation syndrome that includes complete absence of all four limbs and other severe 
anomalies (Niemann et al. 2004). As both ANKRD11 and WNT3 are transcribed on the negative strand, the translocation results in two chimeric genes (Supplementary Fig. 5). Although the chimeric gene at the der(16) breakpoint lacks ANKRD11 exons 1-3, it has an intact ANKRD11 open-reading frame downstream of WNT3 exon 1, translational initiation codon and WNT3 5' regulatory region.

Regarding the gene content of the 16q24.3 breakpoint-spanning TAD (brTAD) in human embryonic stem cells (hESC) (Dixon et al. 2012), the mitochondrial metalloprotease protein coding gene, SPG7 (paraplegin matrix AAA peptidase subunit) associated with AR/AD adult-onset spastic paraplegia 7 (SPG7, OMIM \#607259) is localized $89 \mathrm{~kb}$ from the breakpoint (Supplementary Fig. 3 and Supplementary Table 8) (Sánchez-Ferrero et al. 2013). Concerning the 17q21.31 in the brTAD (Fig. 3), in addition to the disrupted WNT3, the myosin light chain 4 gene (MYL4) is localized $425 \mathrm{~kb}$ distal to the breakpoint and is etiologic in dominant atrial fibrillation, familial, 18 (ATFB18, OMIM \#617280) with an age of onset of 35 years (Orr et al. 2016). Further distal in the brTAD is ITGB3 or platelet glycoprotein IIIa, which has been reported to cause AR or AD platelet-related mild bleeding disorders (BDPLT16, OMIM \#187800).

In DGRC0019, the 2p13.3 breakpoint at position chr2:70,941,502 disrupts IVS1 of ATP6V1B1 (ATPase H+ transporting V1 subunit B1, OMIM *192132), whereas the 19q13.11 breakpoint at position chr19:32,878,515 is located within the 3' UTR of the CEP89 transcript NM_032816 (centrosomal protein 89, OMIM *615470) (Fig. 2). The disrupted ATPase is a component of the vacuolar ATPases, a multi-subunit enzyme that mediates acidification of eukaryotic intracellular organelles. Pathogenic variants within this gene are reported to cause an AR distal renal tubular acidosis with progressive nerve deafness (잎 \#267300) (Karet et al. 1999). A homozygous deletion comprising CEP89 and SLC7A9 has been reported in a patient with isolated mitochondrial complex IV deficiency, intellectual disability and multisystemic problems (van Bon et al. 2013). SLC7A9, causing cystinuria (OMIN \#220100) with AR and AD inheritance with incomplete penetrance, was identified within the hESC and LCL GM12878 brTADs $8.75 \mathrm{~kb}$ proximal 
from the 19q13.11 breakpoint (Supplementary Fig. 6d and Supplementary Tables 9 and 10) (Rao et al. 2014; Leclerc et al. 2002).

In DGRC0006, the 8q12.3 breakpoint disrupts IVS1 of a large intergenic non-coding (Linc) RNA LINC01414 or RP11-32K4.1 with a brain-specific expression pattern and unreported biological function (Supplementary Fig. 7). The 14q31.2 breakpoint is in a large gene poor region.

In DGRC0013, IVS1 of FLT1 (Fms related tyrosine kinase 1, OMIM *165070) is disrupted (Supplementary Fig. 8). FLT1 is a tyrosine kinase receptor for vascular endothelial growth factors (VEGF) with important roles in angiogenesis and vasculogenesis. Although this receptor has been implicated in development and homeostasis of many organs, it is not yet associated with a human disorder (Tjwa et al. 2003). Flt1 knockout mice models show increased angiogenesis, left ventricle wall thickening and enlargement of the left ventricle cavity, only the last of which is consistent with the DGRC0013 phenotype (Fong et al. 1995; Mei et al. 2015). However, it is not unsurprising that a disruption of a single allele in FLT1 is not totally representative of the loss-of function phenotype in the knockout mouse. No Flt1 knockout mice study showed abnormalities of the tricuspid valve as did DGRC0013, but the repression of VEGF was described as part of the mechanism for heart valve morphogenesis (Chang et al. 2004). Moreover, the enhanced expression of FLT1 in atrioventricular valves, per FANTOM CAT browser, correlates with the reported valve abnormality in the patient (Hon et al. 2017).

$$
\text { In DGCR0025, ANKSIB (Ankrd11 repeat domain 11, OMIM *611192) IVS9 is }
$$
disrupted (Supplementary Fig. 9). ANKS1B is a tyrosine kinase effector of activity-dependent post-synaptic signaling and a component of the postsynaptic density complex (Jordan et al. 2007). ANKS1B shows an enriched brain-specific expression pattern. Recently, monogenic heterozygous microdeletions in $A N K S 1 B$ have been reported to cause a spectrum of neurodevelopmental phenotypes (Carbonell et al. 2019).

Finally, in DGRC0030 the breakpoints disrupt exon 12 of WDR26 (WD repeatcontaining protein 26 ; $\underline{\mathrm{OMIM} * 617424)}$ ) and IVS1 of ATP2B2 (ATPase plasma membrane 
Ca2+ transporting 2; OMIM *108733) (Supplementary Fig. 10). Pathogenic variants in these genes are reported to cause AD SKDEAS and AD non-syndromic sensorineural hearing impairment, respectively (Skraban et al. 2017; Smits et al. 2019).

\section{Genomic imbalances}

The median size of del and dup at clinical resolution is 64 and $49 \mathrm{~kb}$, respectively. Two deletions, $53.512 \mathrm{~kb}$ at $3 \mathrm{p} 24.1(27,354,680-27,408,191)$ and $836.049 \mathrm{~kb}$ at $8 \mathrm{q} 24.21$ $(129,061,233-129,897,281)$, identified in DGRC0016 (Supplementary Figs. 11, 12 and Supplementary Table 11) were not found in public CNV databases. The deletion at 3p24.1, classified as a VUS with a total score of $-0.45(1 \mathrm{~A}, 3 \mathrm{~A}, 4 \mathrm{~J}(-0.30), 5 \mathrm{C}(-0.15))$ according to ACMG CNV criteria (Riggs et al. 2019), is present in the proband's phenotypically normal mother and brother, and therefore unlikely to contribute to an abnormal phenotype. As for the $836.047 \mathrm{~kb}$ de novo deletion, none of the affected genes has been considered to cause a reported phenotype and the deletion is interpreted as VUS according to ACMG CNV criteria (Riggs et al. 2019). Moreover, although several genetic traits have been associated by GWAS with the affected genomic region, none of these represent developmental disorders (Supplementary Table 12). Posteriorly, this SV was considered as unrelated to the patient's reported clinical features. Regarding DGRC0019, with the exception of a 12,033 bp deletion within the olfactory receptor family 5 subfamily B pseudogene region (chr11q12.1:58336732-58348764), no other proband-specific alteration was detected (Supplementary Table 13).

Proband-specific del and dup identified in the retrospectively analyzed probands are summarized in Supplementary Table 14, and inv, ins and cx SV in Supplementary Table 15. Most likely, none of these SVs has a pathogenic implication.

\section{Expression studies}

From the disrupted genes in prenatal probands, only ANKRD11 and CEP89 are ubiquitously expressed in LCLs. WNT3 shows skin enriched expression, whereas ATP6V1B1 has kidney, lung and skin enhanced expression (Supplementary Figs. 13 and 14). Expression array profiling of the $t(16 ; 17)$ proband's LCLs shows that due to low sensitivity of this HTA 2.0 
array, the whole gene expression level of $A N K R D 11$ is roughly the same as that in controls (7.77 vs. 7.85, SD 0.09$)$ whereas that of WNT3 is increased (5.86 vs. 4.6, SD 0.01) (Supplementary Table 16). The increased WNT3 exon 5 signal intensity (33.58) may explain the observed whole gene elevated WNT3 expression (Supplementary Fig. 15). Expression levels of the remaining genes from both brTAD were roughly similar to controls (Supplementary Table 16).

HTA 2.0 expression data of cultured human amniocytes are not available in the literature. Therefore, expression data of the $\mathrm{t}(2 ; 19)$ proband's cultured amniocytes were compared to LCLs as control. Noticeable altered expression above the threshold of the microarray was not observed at the level of gene, exon or exon splicing (data not shown).

\section{Predictability of the phenotypic outcome of dnBCA}

The pathogenicity of an SV should be assessed separately for each breakpoint and jointly as a single alteration. In the absence of established guidelines or criteria for classification of SV, we based our classification on ACMG criteria for sequence variants (Richards et al. 2015). Variant classification and clinical interpretation of BCAs is summarized in Table 1.

During PND of DGRC0016, the $\mathrm{t}(16 ; 17)$ rearrangement at 16q24.3 was classified as PM6 (ACMG criterion PM6 - assumed de novo, but without confirmation of paternity and maternity) and the absence of ANKRD11 exons 1-3 in the der(16) as PSV1 (Table 1). Therefore, the $16 \mathrm{q} 24.3$ rearrangement was interpreted as a likely pathogenic variant, most likely leading to a KBG syndrome-like phenotype. Postnatally, it was further classified as PP4 (PP4 - patient's phenotype and family history highly specific for a disease with a single gene etiology). Moreover, although KBG syndrome is typically milder and less frequently diagnosed in females, the patient's clinical features meet the diagnostic criteria for KBG (Richards et al. 2015) (Supplementary Table 2) and therefore the ACMG interpretation was upgraded to pathogenic (Table 1).

During PND of DGRC0019, the $\mathrm{t}(2 ; 19)$ rearrangement at 2p13.3 was also classified as PM6 and according to our interpretation criteria (Table 1) was predicted to have a "Low potential of disease." 
For DGRC0006, none of the affected genes or identified genomic alterations is associated with pathologies nor show overlap with the patient's phenotype. Furthermore, GWAS data do not reach genome-wide statistical significance (Supplementary Table 17). Therefore, based on our criteria we consider this variant as "Non-disease causing" (Table 1).

In DGRC0013 and DGRC0025, dnBCA breakpoints directly disrupt genes with a low ratio of observed / expected (oe) number of LoF variants indicating a strong LoF intolerance (Table 1 and Supplementary Tables 18 and 19) but neither are curated in ClinGen. Both SVs (i.e., involving FLT1 and ANKS1B) can only be scored to PM6, but based on our criteria are predicted to be "Disease plausible" (Table 1). Of note, the clinical phenotype of DGRC0025 matches a recently reported $A N K S 1 B$ haploinsufficiency syndrome (Carbonell et al. 2019).

Finally, in DGRC0030, the SV disrupting the disease gene WDR26 was classified as PVS1, PM6 and PP4 corresponding to pathogenic by ACMG criteria (Richards et al. 2015). Thus, for the WDR26 variant our interpretation was "Disease causing" and the proband's clinical phenotype coincides with that of age-matched patients with SKDEAS (Skraban et al. 2017). $A T P 2 B 2$ is not curated as a dominant disease locus (Supplementary Table 20), but the $A T P 2 B 2$ variant is classified as "Disease plausible" (Table 1).

\section{TAD analysis}

It is now clearly established in the literature that disruption of TADs and the creation of neoTADs are dominant mechanisms of SVs (Lupiáñez et al. 2015; Franke et al. 2016). The main source of knowledge of TAD maps are generated by chromosome conformation capture (HiC) data. Details of TADs involved in DGRC0016 are presented in Fig. 3. None of the $\mathrm{t}(16 ; 17)$ breakpoints disrupt an interaction loop (data not shown) (Rao et al. 2014). Hi-C contact heatmaps of the $\mathrm{t}(2 ; 19)$ breakpoint regions for LCLs and IMR90 are shown in Supplementary Fig. 6. Although the 2p13.3 breakpoint disrupts two interaction loops (Supplementary Fig. 6ab) (Rao et al. 2014), none of the involved genes shows LoF sensitivity. 


\section{DISCUSSION}

Genome sequencing data of two ongoing fetal and four retrospective samples with dnBCA identified during conventional PND were analyzed by liGS, followed by comprehensive structural analyses of candidate genes from the disrupted bpTADs and prediction of the phenotypic outcome. Moreover, to facilitate implementation of this analysis, two new bioinformatic tools applicable in the clinical setting have been developed. Using this information and the developed bioinformatic tools, we propose an analytical workflow for identification and interpretation of de novo SVs in their genomic landscape (Fig. 4).

In DGCR0016, translocation breakpoints disrupt a single allele of ANKRD11 and of WNT3, wherein haploinsufficiency of ANKRD11 causes AD KBGS. KBGS was first reported by Herrmann et al. (1975) in three unrelated families with the surnames initials being $\mathrm{K}, \mathrm{B}$ and $\mathrm{G}$. The common phenotypic characteristics of this multiple congenital anomaly comprises, among others, a characteristic facial appearance (including protruding ears and hypertelorism), hand anomalies, neurologic involvement, and postnatal short stature (Skjei et al. 2007), which are consistent with the phenotype observed in the patient and fits KBGS diagnostic criteria (Low et al. 2016).

In DGCR0019, translocation breakpoints disrupt genes tolerant to LoF variants, ATP6V1B1 and CEP89. No gene causing AD or developmental disorder was identified within the bpTADs. The predicted outcome was confirmed by absence of a postnatal clinical phenotype. Nevertheless, longer term follow-up would be warranted to exclude any later onset of a disorder that might be associated as recently demonstrated for prenatally detected dnBCAs (Halgren et al. 2018) or natural history of individuals with postnatal dnBCAs (Currall et al. 2018).

Of the four retrospectively analyzed dnBCAs, similarly to the aforementioned DGCR0016, disruption of WDR26 predictably will lead to SKDEAS. The patient's clinical phenotype highlights that these phenocopies, SKDEAS and 1q41q42 deletion syndrome, are primarily caused by disruption of WDR26. 
Although the breakpoints of $\operatorname{dnBCAs} \operatorname{inv}(13)$ and $t(12 ; 17)$ disrupt genes not yet curated in ClinGen, the fact that these are significantly LoF intolerant genes involved in several biological processes, reinforced by convergent evidence, led us to predict that they are "Disease plausible". Furthermore, DGRC0025 clinical phenotype overlaps ANKS1B haploinsufficiency syndrome.

Finally, for the postnatal phenotype of DGRC0006, the $t(8 ; 14)$ variant is predicted as "Non-disease causing." Although pathogenic cx SVs smaller than our clinical resolution cutoff have been reported (Sanchis-Juan et al. 2018), at the higher resolution of liGS no additional presumably pathogenic SV was identified in DGRC0006. Exome sequencing (ES) has not been performed, and other non-genetic factors unrelated to the translocation may be responsible for the phenotype (e.g., environmental or multifactorial factors).

Short-read sequencing by either genome sequencing (GS) or ES has been applied in the prenatal setting. ES in fetuses with structural anomalies was recently elucidated in a largescale study (Lord et al. 2019; Petrovski et al. 2019) revealing a genetic etiology in about 10\% of affected fetuses. However, short-read sequencing is not optimal for identification of SVs. The physical coverage of GS is relatively low, whereas ES is high but will miss breakpoints localized within non-coding sequence. The long-insert size of the liGS libraries, intended for identification of BCAs and CNVs, and low read size results in high physical but low sequence coverage.

The lack of transcriptome data on gestational age- and sex-matched first trimester cells from CVS and amniotic fluid is a current limitation for introduction of gene expression analysis in the clinical prenatal setting. Clearly, the future of prenatal diagnosis for SVs will require generation of gene expression data by RNA-Seq linked to Hi-C of CVS cells and amniocytes, as is available now in public databases for adult tissues. Presently, the interpretation of current PND of dnBCAs could be limited to disruption of major dominant genes leading to Mendelian disorders as occurred for ANKRD11, WDR26 and ANKS1B. Nonetheless, cytogenetics laboratories should be attentive to take into consideration the 
architectural features of genomes to address fully the disease potential of a SVs (Lupiáñez et al. 2015).

In comparison to karyotyping and CMA analysis, we demonstrate the benefits of an liGS-based approach and our clinically inspired pipeline for identification of dnBCA breakpoints and interpretation of the genomic landscape on which these occurred in the prenatal setting. We show the predictability of the clinical outcome of these BCAs and plan to provide updated bioinformatic tools to facilitate data analysis and a workflow for implementation of genome sequencing in the diagnostic prenatal setting. 


\section{Compliance with Ethical Standards}

Conflict of interest: The authors declare no conflicts of interest.

Informed consent: This study was approved by the Ethics Committee of the National Institute of Health Doutor Ricardo Jorge and was carried out according to the Principles of the Declaration of Helsinki of the World Medical Association. Samples were obtained after written informed consent of the participants or their legal representatives. Additional informed consent was obtained from all individual participants for whom identifying information is included in this article. 


\section{References}

Chen W, Kalscheuer V, Tzschach A, et al. (2008) Mapping translocation breakpoints by nextgeneration sequencing. Genome Res 18:1143-1149.

Carbonell AU, Cho CH, Tindi JO, et al. (2019) Haploinsufficiency in the ANKS1B gene encoding AIDA-1 leads to a neurodevelopmental syndrome. Nat Commun 10:3529.

Chang CP, Neilson JR, Bayle JH, et al. (2004) A field of myocardial-endocardial NFAT signaling underlies heart valve morphogenesis. Cell 118:649-663.

Collins RL, Stone MR, Brand H, et al. (2016) CNView: a visualization and annotation tool for copy number variation from whole-genome sequencing. bioRxiv 049536; doi: https://doi.org/10.1101/049536.

Collins RL, Brand H, Redin CE, et al. (2017) Defining the diverse spectrum of inversions, complex structural variation, and chromothripsis in the morbid human genome. Genome Biol 18:36.

Currall BB, Chen M, Sallari RC, et al. (2018) Loss of LDAH associated with prostate cancer and hearing loss. Hum Mol Genet 27:4194-4203.

David D, Cardoso J, Marques B, et al. (2003) Molecular characterization of a familial translocation implicates disruption of HDAC9 and possible position effect on TGFB2 in the pathogenesis of Peters' anomaly. Genomics 81:489-503.

Dixon JR, Selvaraj S, Yue F, et al. (2012) Topological domains in mammalian genomes identified by analysis of chromatin interactions. Nature 485:376-380.

Franke M, Ibrahim DM, Andrey G, et al. (2016) Formation of new chromatin domains determines pathogenicity of genomic duplications. Nature 538:265-269.

Fong GH, Rossant J, Breitman ML. (1995) Role of the FLT1 receptor tyrosine kinase in regulating the assembly of vascular endothelium. Nature 376:66-69.

Halgren C, Nielsen NM, Nazaryan-Petersen L, et al. (2018) Risks and recommendations in prenatally detected de novo balanced chromosomal rearrangements from assessment of long-term outcomes. Am J Hum Genet 102:1090-1103. 
Herrmann J, Pallister PD, Tiddy W, Opitz JM. (1975) The KBG syndrome: A syndrome of short stature, characteristic facies, mental retardation, macrodontia and skeletal anomalies. Birth Defects Orig Artic 11:7-18.

Hon CC, Ramilowski JA, Harshbarger J, et al. (2017) An atlas of human long non-coding RNAs with accurate 5' ends. Nature 543:199-204.

Jordan BA, Fernholz BD, Khatri L, Ziff EB. (2007) Activity-dependent AIDA-1 nuclear signaling regulates nucleolar numbers and protein synthesis in neurons. Nat Neurosci 10:427-435.

Karet FE, Finberg KE, Nelson RD, et al. (1999) Mutations in the gene encoding B1 subunit of H+-ATPase cause renal tubular acidosis with sensorineural deafness. Nat Genet 21:84-90.

Klambauer G, Schwarzbauer K, Mayr A, et al. (2012) cn.MOPS: mixture of Poissons for discovering copy number variations in next-generation sequencing data with a low false discovery rate. Nucleic Acids Res 40:e69.

Leclerc D, Boutros M, Suh D, et al. (2002) SLC7A9 mutations in all three cystinuria subtypes. Kidney Int 62:1550-1559.

Lord J, McMullan DJ, Eberhardt RY, et al. (2019) Prenatal exome sequencing analysis in fetal structural anomalies detected by ultrasonography (PAGE): a cohort study. Lancet 393:747757.

Low K, Ashraf T, Canham N, et al. (2016) Clinical and genetic aspects of KBG syndrome. Am J Med Genet A 170:2835-2846.

Lupiáñez DG, Kraft K, Heinrich V, et al. (2015) Disruptions of topological chromatin domains cause pathogenic rewiring of gene-enhancer interactions. Cell 161:1012-1025.

Mei L, Huang Y, Lin J, et al. (2015). Increased cardiac remodeling in cardiac-specific Flt-1 receptor knockout mice with pressure overload. Cell Tissue Res 362:389-398.

Moore BL, Aitken S, Semple CA. (2015) Integrative modeling reveals the principles of multiscale chromatin boundary formation in human nuclear organization. Genome Biol 16:110.

Niemann S, Zhao C, Pascu F, et al. (2004) Homozygous WNT3 mutation causes tetra-amelia in a large consanguineous family. Am J Hum Genet 74:558-563. 
Ordulu Z, Wong KE, Currall BB, et al. (2014) Describing sequencing results of structural chromosome rearrangements with a suggested next-generation cytogenetic nomenclature. Am J Hum Genet 94:695-709.

Ordulu Z, Kammin T, Brand H, et al. (2016) Structural chromosomal rearrangements require nucleotide-level resolution: lessons from next-generation sequencing in prenatal diagnosis. Am J Hum Genet 99:1015-1033.

Orr N, Arnaout R, Gula LJ, et al. (2016) A mutation in the atrial-specific myosin light chain gene (MYL4) causes familial atrial fibrillation. Nat Commun 7:11303.

Petrovski S, Aggarwal V, Giordano JL, et al. (2019) Whole-exome sequencing in the evaluation of fetal structural anomalies: a prospective cohort study. Lancet 393:758-767.

Rao SSP, Huntley MH, Durand NC, et al. (2014) A 3D map of the human genome at kilobase resolution reveals principles of chromatin looping. Cell 159:1665-1680.

Redin C, Brand H, Collins RL, et al. (2017) The genomic landscape of balanced cytogenetic abnormalities associated with human congenital anomalies. Nat Genet 49:36-45.

Richards S, Aziz N, Bale S, et al. (2015) Standards and guidelines for the interpretation of sequence variants: a joint consensus recommendation of the American College of Medical Genetics and Genomics and the Association for Molecular Pathology. Genet Med 17:405424.

Riggs ER, Andersen EF, Cherry AM, et al. (2019) Technical standards for the interpretation and reporting of constitutional copy-number variants: a joint consensus recommendation of the American College of Medical Genetics and Genomics (ACMG) and the Clinical Genome Resource (ClinGen). Genet Med; doi: 10.1038/s41436-019-0686-8.

Sánchez-Ferrero E, Coto E, Beetz C, et al. (2013) SPG7 mutational screening in spastic paraplegia patients supports a dominant effect for some mutations and a pathogenic role for p.A510V. Clin Genet 83:257-262.

Sanchis-Juan A, Stephens J, French CE, et al. (2018) Complex structural variants in Mendelian disorders: identification and breakpoint resolution using short- and long-read genome sequencing. Genome Med 10:95. 
Sirmaci A, Spiliopoulos M, Brancati F, et al. (2011) Mutations in ANKRD11 cause KBG syndrome, characterized by intellectual disability, skeletal malformations, and macrodontia. Am J Hum Genet 89:289-294.

Skjei KL, Martin MM, Slavotinek AM (2007) KGB syndrome: report of twins, neurological characteristics, and delineation of diagnostic criteria. Am J Med Genet 143A:292-300.

Skraban CM, Wells CF, Markose P, et al. (2017) WDR26 haploinsufficiency causes a recognizable syndrome of intellectual disability, seizures, abnormal gait, and distinctive facial features. Am J Hum Genet 101:139-148.

Smits JJ, Oostrik J, Beynon AJ, et al. (2019) De novo and inherited loss-of-function variants of ATP2B2 are associated with rapidly progressive hearing impairment. Hum Genet 138:6172.

Talkowski ME, Ernst C, Heilbut A, et al. (2011) Next-generation sequencing strategies enable routine detection of balanced chromosome rearrangements for clinical diagnostics and genetic research. Am J Hum Genet 88:469-481.

Talkowski ME, Ordulu Z, Pillalamarri V, et al. (2012) Clinical diagnosis by whole-genome sequencing of a prenatal sample. N Engl J Med 367:2226-2232.

Tjwa M, Luttun A, Autiero M, Carmeliet P. (2003) VEGF and PIGF: two pleiotropic growth factors with distinct roles in development and homeostasis. Cell Tissue Res 314:5-14. van Bon BW, Oortveld MA, Nijtmans LG, et al. (2013) CEP89 is required for mitochondrial metabolism and neuronal function in man and fly. Hum Mol Genet 22:3138-3151. 


\section{Figure legends}

Fig. 1 Ideograms, partial karyotype and liGS-based localization of the $t(16 ; 17)(\mathrm{q} 24.3 ; \mathrm{q} 21.3) \mathrm{dn}$ breakpoints at genomic and gene levels. a, b Ideograms and GTL-banded normal and derivative metaphase chromosomes. Chromosome 17 ideogram is shaded in yellow. Beside the derivative ideograms the karyotype and liGS-based resolution of the breakpoints are specified. Filled diamonds or arrows indicate chromosome breakpoints. Karyotype resolution indicates the size of the identified disrupted chromosome band. $\mathbf{c}, \mathbf{d}$ Localization of the $\mathrm{t}(16 ; 17)(\mathrm{q} 24.3 ; \mathrm{q} 21.3) \mathrm{dn}$ breakpoints at genomic and gene level based on the translocation-specific chimeric cluster. Black and blue arrowheads depict chimeric reads aligned to chromosomes 16 and 17 , respectively. Below, gene structure of the disrupted genes, reference transcript numbers and the translational initiation codons (ATG) are indicated. A split-read between positions chr17:46,781,986 and chr16:89,401,732 identified at the der(17) breakpoint is shown by a double arrowhead.

Fig. 2 Ideograms, partial karyotype and liGS-based localization of the $t(2 ; 19)(p 13.3 ; q 13.11) d n$ breakpoints at genomic and gene levels. a, b Ideograms and GTL-banded normal and derivative metaphase chromosomes. Chromosome 19 ideogram is shaded in yellow. Beside the derivative ideograms the karyotype and liGS-based resolution of the breakpoints are specified. Filled diamonds or arrows indicate chromosome breakpoints. Karyotype resolution indicates the size of the disrupted chromosome band established by metaphase analysis. c, $\mathbf{d}$ Localization of the $\mathrm{t}(2 ; 19)(\mathrm{p} 13.3 ; \mathrm{q} 13.11) \mathrm{dn}$ breakpoints at genomic and gene levels based on the translocationspecific chimeric cluster. Black and blue arrowheads depict chimeric reads aligned to chromosomes 2 and 19, respectively. Below, gene structure of the disrupted ATP6B1, reference transcript number and the translational initiation codon are indicated. Additionally, the genomic position of the 19q13.1 breakpoint within the 3'UTR of CEP89 is shown. 
Fig. 3 TADs spanning translocation breakpoints of t(16;17) in LCL GM12878, IMR90, and hESC, and chimeric TADs from derivative chromosomes. a 16q24 breakpoint region. b 17q21.3 breakpoint region. Chromosome 16 TADs are depicted in black or gray, whereas those of chromosome 17 in blue or light blue. Below the TAD tracks, CCCTC-binding factor (CTCF) sites from the analyzed region are shown according to the Chip-seq track in IMR90 fibroblasts (IMR90 CTCF IgG-rab ChIP-seq Signal from ENCODE/SYDH; ENCODE Project Consortium). Horizontal lines with folded gray arrowheads indicate the position of genes in sense and antisense orientations. Genes are color-coded according to their haploinsufficiency index (HI) available at https://decipher.sanger.ac.uk/ and their LoF intolerance, expressed as oeratio of LoF variants stated below the genes (http://gnomad.broadinstitute.org/). ANKRD11 causing KBGS is marked with a hash mark (\#). Data for IMR90 fibroblasts, hESCs and LCL GM12878 are according to Dixon et al. (2012) and Moore et al. (2015), respectively.

Fig. 4 Proposed workflow for nucleotide level resolution and interpretation of de novo structural rearrangements in their genomic landscape in prenatal diagnosis. a In the case of BCAs associated with fetal anomalies or de novo SVs, concomitantly with the conventional PND protocol, liGS should be performed for nucleotide level resolution of the rearrangement breakpoints in their genomic landscape. Inherited BCAs are referred to a clinical geneticist to lay out the follow-up required, including potential inclusion of liGS. Foreseeably, upon improvement of liGS-based methods, all prenatally identified non-polymorphic SV may be analyzed by such an approach.

b Long-insert based genome sequencing such as mate-pair, with short or medium reads, from $2 \mathrm{x}$ 25 to $250 \mathrm{bp}$, can be used. Increasing the sequence coverage enables identification of SNV and indels from the same sequencing data.

c The proposed bioinformatic workflow:

i) Sequence data decoded in different types of read-pairs,

ii) Clusters denoting different types of balanced and unbalanced SVs (translocations, ins, inv, cx $\mathrm{SV}$, del and dup) are identified at the liGS resolution,

iii) Identification of cluster-specific split-reads, 
iv) Genomic regions comprising deletions and tandem duplications revealed by the two procedures merged,

v) Potentially pathogenic candidate genes, genomic loci and CNVs revealed by bioinformatic tools TAD-GConTool and CNV-ConTool, and

vi) Analysis of the disrupted or dysregulated genes and CNVs, orthogonal confirmation, validation, and preparation of report by a certified medical geneticist. 
Table 1 Overview of phenotypes, sequencing results and interpretation, and clinical outcomes of patients with dnBCAs

\begin{tabular}{|c|c|c|c|c|c|c|c|c|}
\hline Subject & $\begin{array}{l}\text { Indication for } \\
\text { PND }\end{array}$ & $\begin{array}{c}\text { Sequence } \\
\text { based } \\
\text { karyotype }\end{array}$ & $\begin{array}{l}\text { Chromo- } \\
\text { some } \\
\text { band }\end{array}$ & $\begin{array}{l}\text { Disrupted } \\
\text { genes }\end{array}$ & $\begin{array}{c}\text { gnomAD } \\
\text { oe }(90 \% \mathrm{Cl})\end{array}$ & $\begin{array}{l}\text { ACMG variant } \\
\text { interpretation }^{a}\end{array}$ & $\begin{array}{c}\text { Clinical } \\
\text { interpretation } \\
\text { and prediction }\end{array}$ & Postnatal clinical phenotype \\
\hline DGRC0016 & $\begin{array}{l}\text { Increased risk for } \\
\text { aneuploidy } \\
\text { following } 1 \text { st } \\
\text { trimester combined }\end{array}$ & $\begin{array}{l}\text { seq[GRCh38] } \\
\mathrm{t}(16 ; 17)(q 24.3 ; q 21 \\
.31) \mathrm{dn}\end{array}$ & $16 q 24.3$ & ANKRD11 & $0.05(0.02-0.11)$ & $\begin{array}{l}\text { Prenatal: Likely } \\
\text { pathogenic, } \\
\text { Posnatal: } \\
\text { Pathogenic }\end{array}$ & Disease causing & \multirow[t]{2}{*}{$\begin{array}{l}\text { KBG syndrome (Supplementary } \\
\text { Table 2) }\end{array}$} \\
\hline & $\begin{array}{l}\text { tests and } \\
\text { increased nuchal } \\
\text { translucency }\end{array}$ & & $17 q 21.31$ & WNT3 & $0.13(0.05-0.39)$ & $\begin{array}{l}\text { Non-disease } \\
\text { associated gene }\end{array}$ & $\begin{array}{l}\text { Non-disease } \\
\text { causing }\end{array}$ & \\
\hline \multirow[t]{2}{*}{ DGRC0019 } & $\begin{array}{l}\text { Maternal anxiety } \\
\text { and advanced }\end{array}$ & $\begin{array}{l}\text { seq[GRCh38] } \\
\mathrm{t}(2 ; 19)(\mathrm{p} 13.3 ; q 13\end{array}$ & $2 p 13.3$ & ATP6V1B1 & $0.63(0.43-0.93)$ & VUS & $\begin{array}{l}\text { Low potential of } \\
\text { disease }\end{array}$ & \multirow[t]{2}{*}{ Healthy newborn } \\
\hline & maternal age & 11)dn & $19 q 13.11$ & CEP89 & $0.93(0.72-1.21)$ & $\begin{array}{c}\text { Non-disease } \\
\text { associated gene }\end{array}$ & $\begin{array}{c}\text { Low potential of } \\
\text { disease }\end{array}$ & \\
\hline \multicolumn{9}{|c|}{ Retrospectively analyzed probands } \\
\hline DGRC0006 & $\begin{array}{l}\text { Advanced } \\
\text { maternal age and } \\
\text { previous child with }\end{array}$ & $\begin{array}{l}\text { seq[GRCh38] } \\
t(8 ; 14)(q 12.3 ; q 31 \\
\text { 2)dn }\end{array}$ & $8 q 12.3$ & $\begin{array}{l}\text { LOC102724 } \\
623 \\
\text { or RP11- } \\
32 K 4.1^{c}\end{array}$ & nd & $\begin{array}{c}\text { Non-disease } \\
\text { associated gene }\end{array}$ & $\begin{array}{l}\text { Non-disease } \\
\text { causing }\end{array}$ & \multirow{2}{*}{$\begin{array}{l}\text { Small for gestational age } \\
\text { (HP:0001518); Failure to thrive } \\
\text { (HP:0001508); Feeding difficulties } \\
\text { (HP:0011968); Moderate global } \\
\text { developmental delay (HP:0011343); } \\
\text { Precocious puberty in females } \\
\text { (HP:0010465) }\end{array}$} \\
\hline & $\begin{array}{l}\text { psychomotor } \\
\text { developmental } \\
\text { delay }\end{array}$ & & $14 q 31.2$ & none & & & $\begin{array}{l}\text { Non-disease } \\
\text { causing }\end{array}$ & \\
\hline \multirow[t]{2}{*}{ DGRC0013 } & $\begin{array}{l}\text { Advanced } \\
\text { maternal age }\end{array}$ & 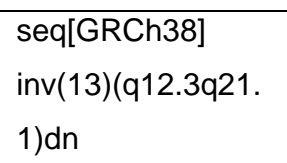 & $13 q 12.3$ & FLT1 & $0.14(0.09-0.24)$ & $\begin{array}{c}\text { Non-disease } \\
\text { associated gene }\end{array}$ & Disease plausible & \multirow{2}{*}{$\begin{array}{l}\text { Infantile muscular hypotonia } \\
\text { (HP:0008947); Moderate global } \\
\text { developmental delay } \\
\text { (HP:0011343);Generalized joint laxity } \\
\text { (HP:0002761); Recurrent upper tract } \\
\text { respiratory infections (HP:0002788); } \\
\text { Bilateral cryptorchidism } \\
\text { (HP:0008689); Phimosis }\end{array}$} \\
\hline & & & $13 q 21.1$ & none & & & $\begin{array}{l}\text { Low potential of } \\
\text { disease }\end{array}$ & \\
\hline
\end{tabular}




\begin{tabular}{|c|c|c|c|c|c|c|c|c|}
\hline \multirow[t]{2}{*}{ DGRC0025 } & $\begin{array}{l}\text { Increased risk for } \\
\text { aneuploidy }\end{array}$ & $\begin{array}{l}\text { seq[GRCh38] } \\
\mathrm{t}(12 ; 17)(q 23.1 ; q 21\end{array}$ & $12 q 23.1$ & $A N K S 1 B$ & $0.10(0.05-0.20)$ & $\begin{array}{c}\text { Non-disease } \\
\text { associated gene }\end{array}$ & Disease plausible & \multirow[t]{2}{*}{$\begin{array}{l}\text { ANKS1B haploinsufficiency } \\
\text { syndrome (Supplementary Table 3) }\end{array}$} \\
\hline & $\begin{array}{l}\text { following } 1 \text { st } \\
\text { trimester combined } \\
\text { tests and } \\
\text { increased nuchal } \\
\text { translucency }\end{array}$ & $.33) d n$ & $17 q 21.33$ & none & & & $\begin{array}{l}\text { Low potential of } \\
\text { disease }\end{array}$ & \\
\hline \multirow[t]{2}{*}{ DGRC0030 } & \multirow[t]{2}{*}{$\begin{array}{l}\text { Advanced } \\
\text { maternal age }\end{array}$} & $\begin{array}{l}\text { seq[GRCh38] } \\
\mathrm{t}(1 ; 3)(\mathrm{q} 42.11 ; \mathrm{p} 25\end{array}$ & $1 q 42.11$ & WDR26 & $0.00(0.00-0.08)$ & Pathogenic & Disease causing & \multirow{2}{*}{$\begin{array}{l}\text { Skraban-Deardorff syndrome } \\
\text { (Supplementary Table 4) } \\
\text { Nonsyndromic sensorineural hearing } \\
\text { impairment }\end{array}$} \\
\hline & & 3)dn & $3 p 25.3$ & ATP2B2 & $0.06(0.03-0.15)$ & $\begin{array}{c}\text { Non-disease } \\
\text { associated gene }\end{array}$ & Disease plausible & \\
\hline
\end{tabular}

${ }^{\mathrm{a} A C M G}$ variant classification according to ClinGen guidelines non-disease genes require first gene curation and then variant classification. ${ }^{\mathrm{b}} \mathrm{Disease}$ and phenotype interpretation categorized as: i) disease causing, ii) disease plausible, iii) low potential of disease, and iv) non-disease causing, the criteria are described in Supplementary Information Intergenic long non-coding RNAs (IncRNAs); The observed / expected (oe) score is from the Genome Aggregation Database (gnomAD; http://gnomad.broadinstitute.org/); nd= no data 
a

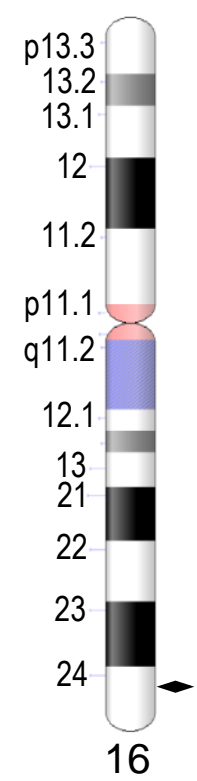

b

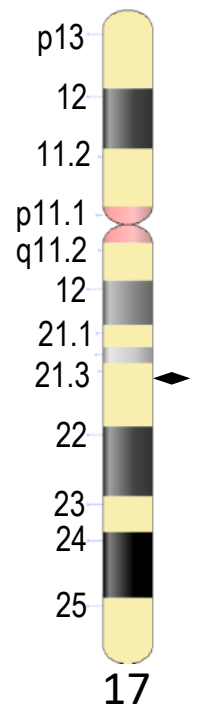

C cen $16 q 11.2$

$89,401,715,46,784,035$

$17 q 11.2$

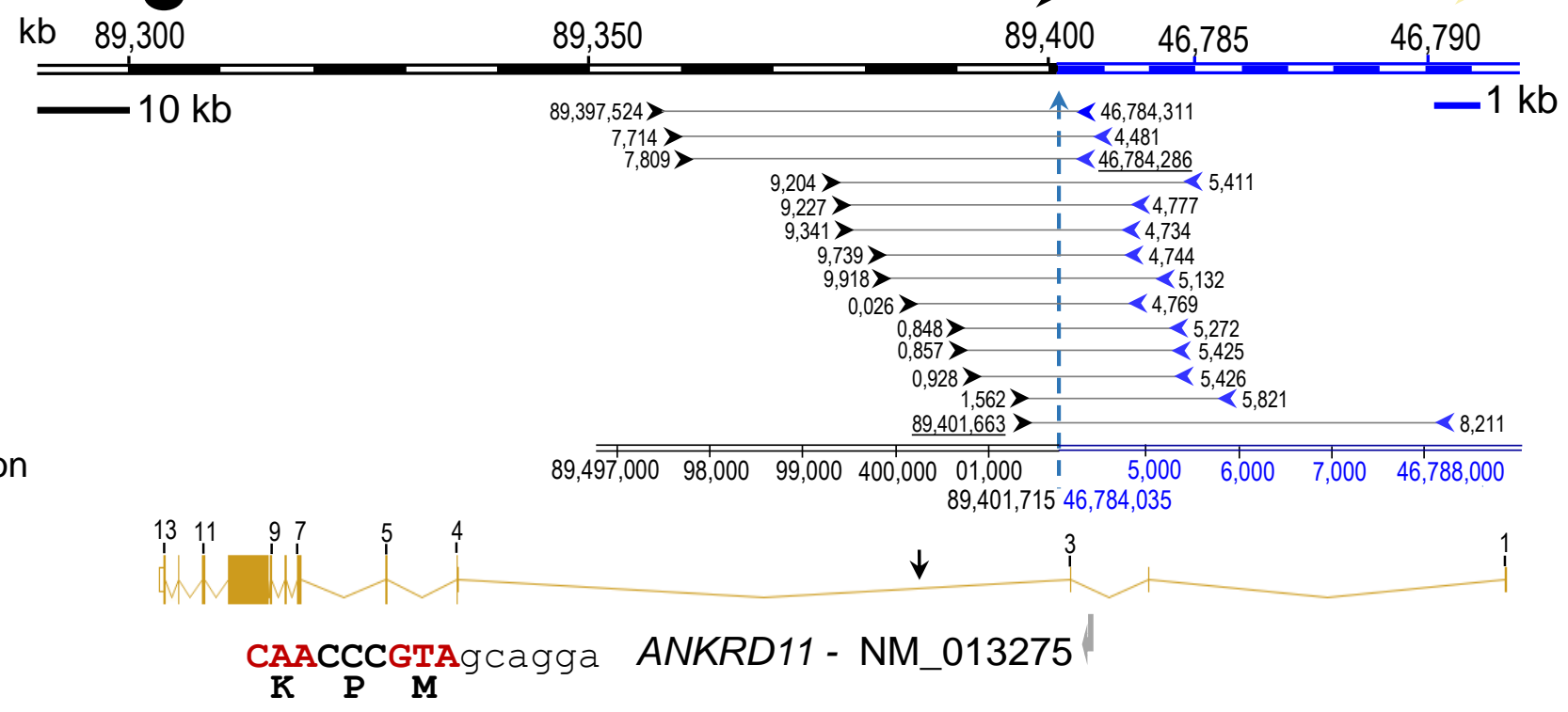

cen $17 q 11.2$

$46,781,99889,401,718$ 16q24.3

$\operatorname{der}(16)$
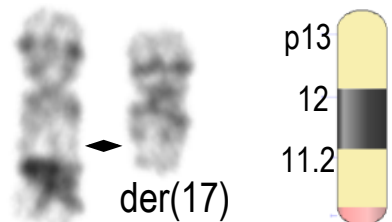

q11.2

Karyotype resolution 21 $4.9 \mathrm{Mb} \rightarrow \underset{\mathrm{q}}{\mathrm{q}} 24.3$

der(17)

liGS

resolution

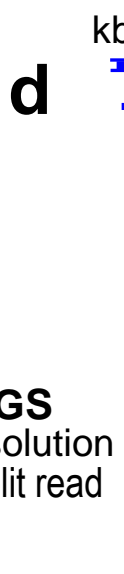

kb 46,765

46,770

46,775

$46,780 \quad 89,400$

89,450

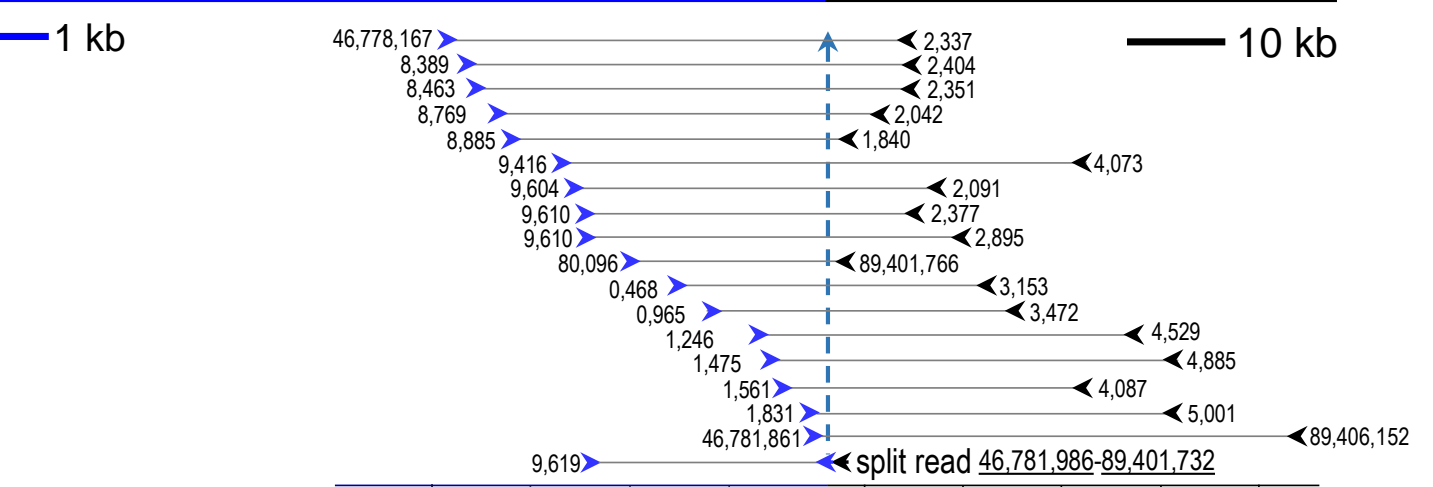

\begin{tabular}{lllllllll}
\hline \hline $46,778,000$ & 79,000 & 80,000 & 81,000 & 2,000 & 3,000 & 4,000 & 5,000 & $89,406,000$
\end{tabular} $46,781,99889,401,718$

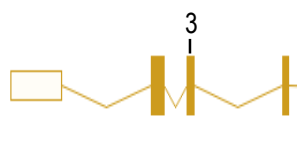


a

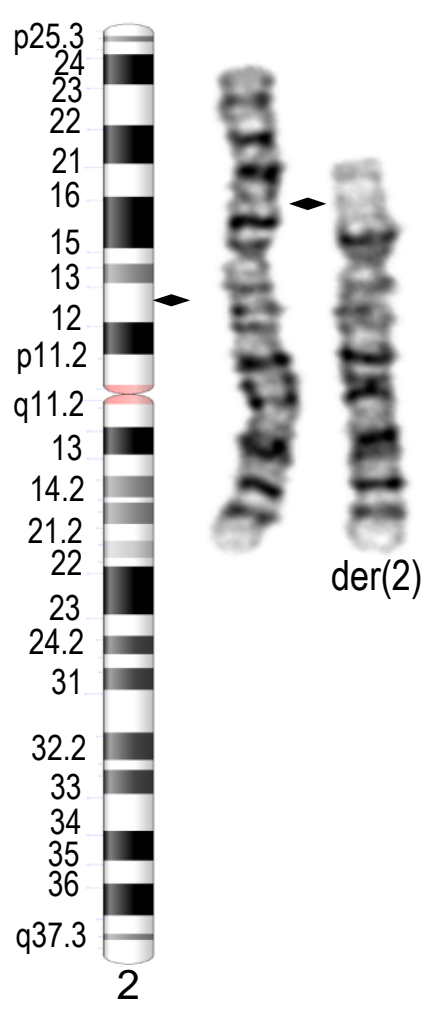

C

19q13.4

$32,878,515 \underline{70,941,507}$

$2 \mathrm{p} 11.2$

cen

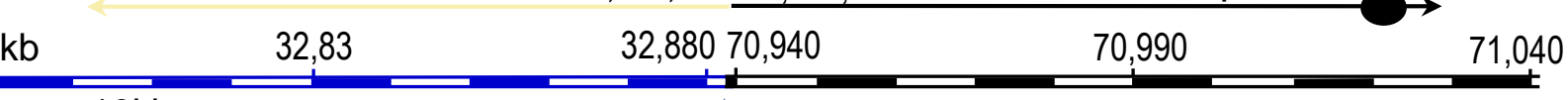

resolution

$6.4 \mathrm{Mb} \overrightarrow{\mathrm{p}} 3_{3} .3$

p11.2

q13.4 liGS

13.2 resolution

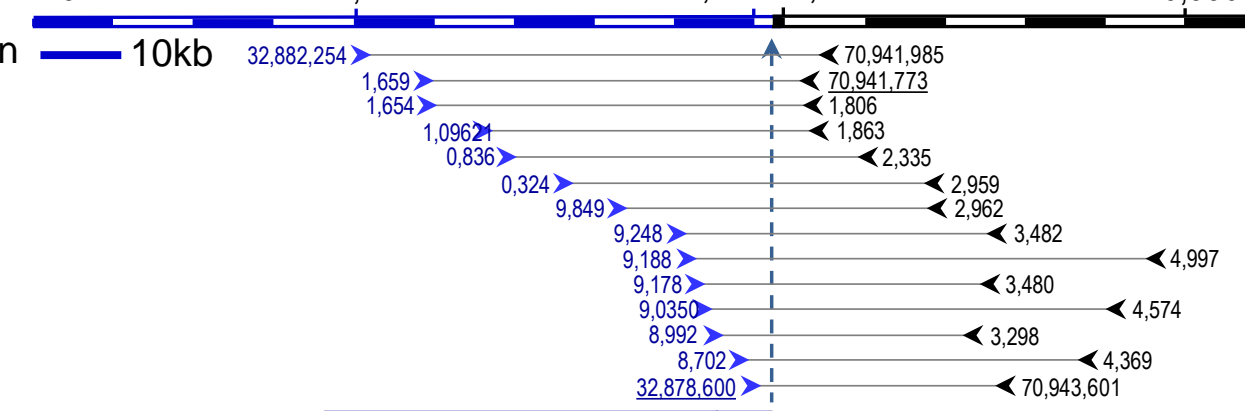

13

14.2

21.2

22

31

$\mathrm{kb} \overline{32,882.0}$

$32,878,600>$

$<70,943,60$

$\begin{array}{lllllll}32,881.0 & 32,880.0 & 32,879.0 & 70,942.0 & 70,943.0 & 70,944.0 & 70,945.0\end{array}$

32.2

33

34

36

q37.3 $32,878,515 \quad 70,941,507$

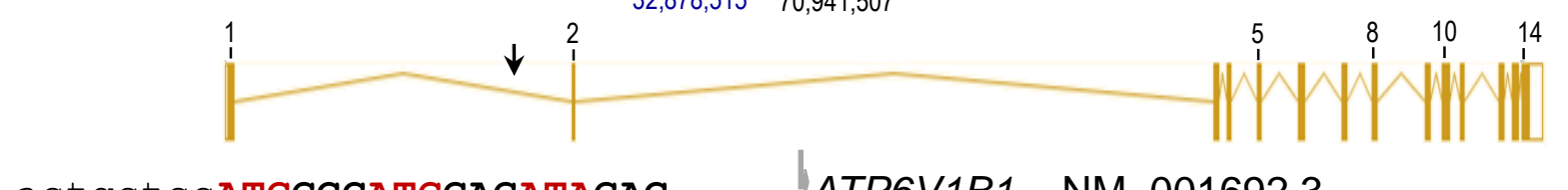

actgct CCATGGCCATGGAGATAGAC

$\begin{array}{llllll}\mathbf{M} & \mathbf{A} & \mathbf{M} & \mathbf{E} & \mathbf{I} & \mathbf{D}\end{array}$

$\operatorname{der}(2)$

cen $19 q 12$

$32,878,51270,941,502$

$2 \mathrm{p} 25.3$

$\mathrm{kb} \quad 32,830 \quad 32,88070,940$ 70,990

b
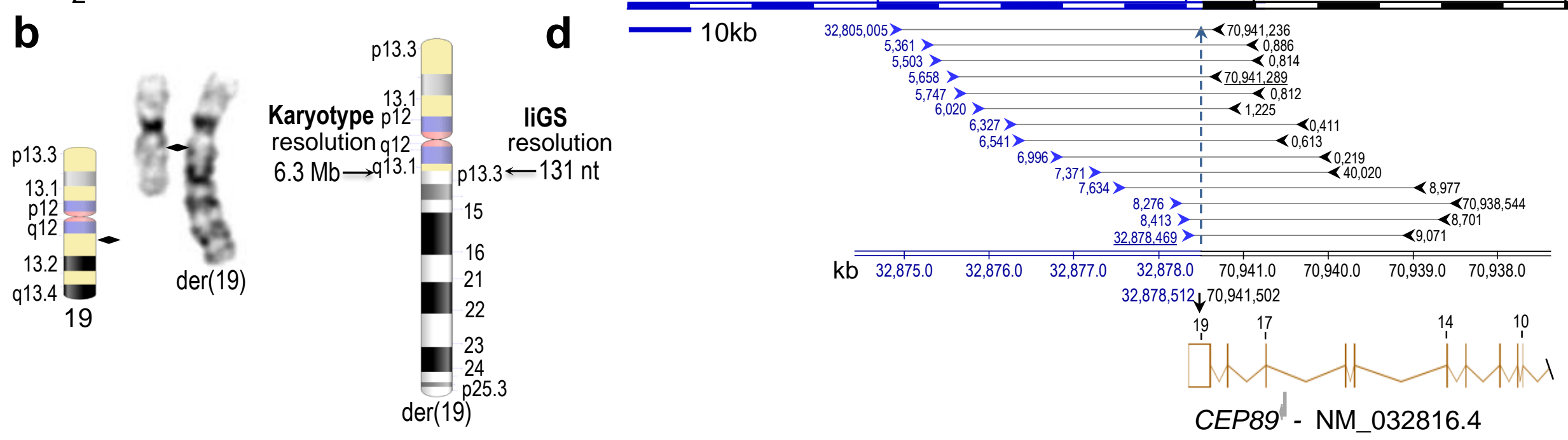
a

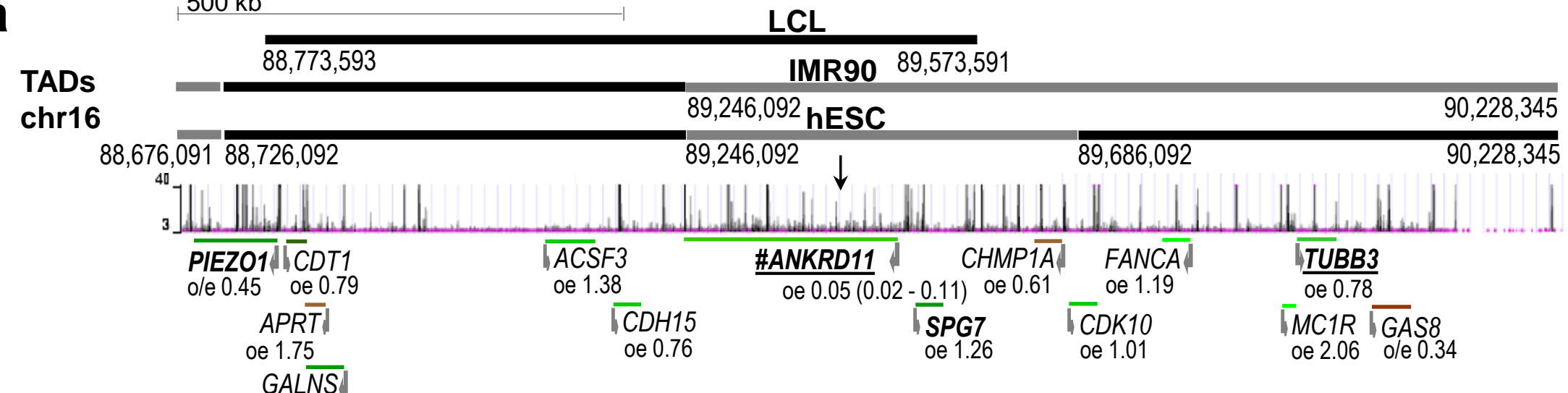

$\operatorname{der}(16)$

hESC $89,401,717-46,784,035$
$88,676,091$
PIEZ01 $\mid \bar{C} D T 1$
APRT
GALNS
\ACSF3 \#ANKRD11WNT3
$\mathrm{CDH} 15$
GOSR2ㅍ
$47,647,635$ 47,807,636 $\sqrt{M Y L 4}$

b

TADs chr17

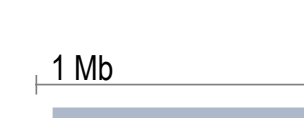

(2)

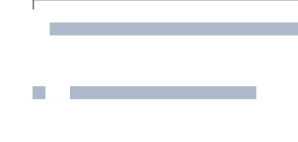

$\mathrm{LCL}$

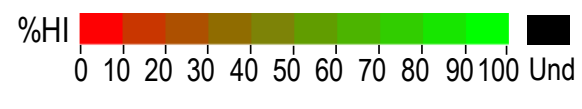

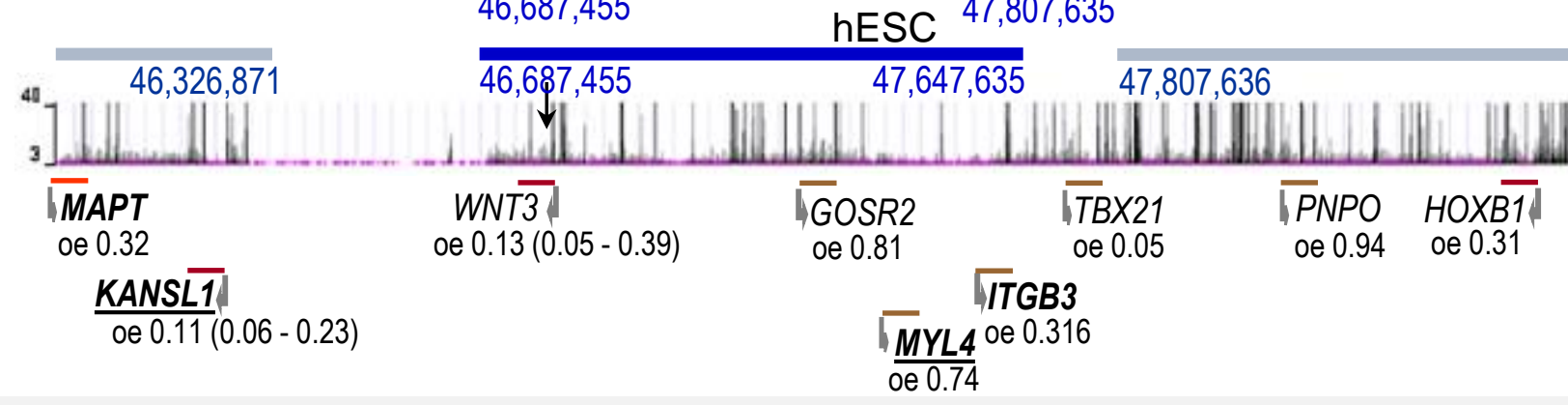

$\operatorname{der}(17)$

hESC $46,784,034-89,401718$

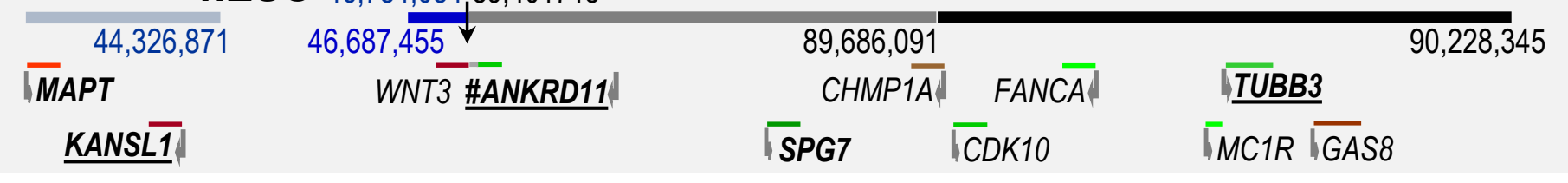




\section{a Conventional prenatal diagnosis}

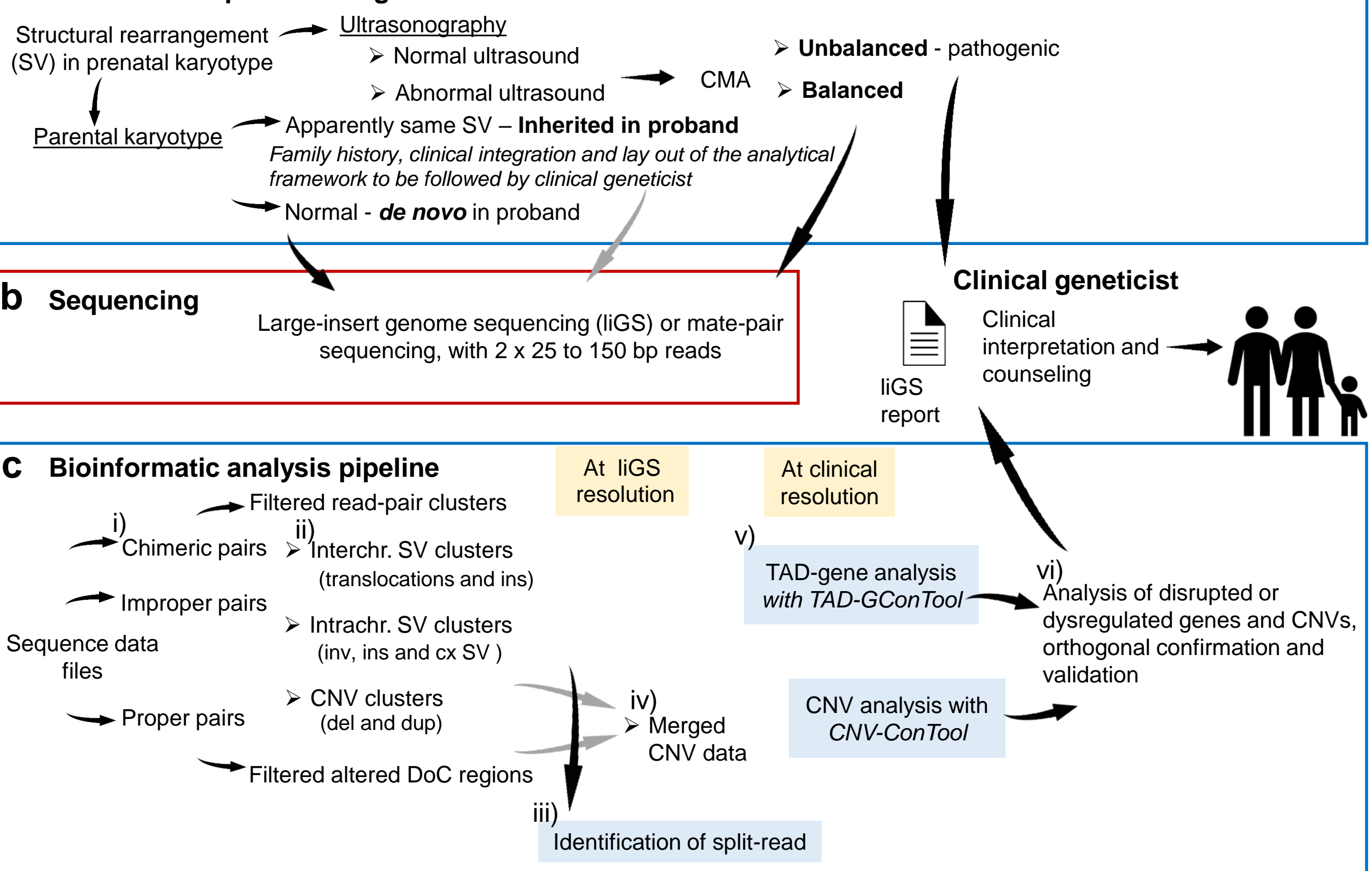




\section{SUPPLEMENTARY MATERIAL}

Comprehensive clinically oriented workflow for nucleotide level resolution and interpretation in prenatal diagnosis of de novo apparently balanced chromosomal translocations in their genomic landscape

Dezső David ${ }^{1}$ João P. Freixo ${ }^{*}$ Joana Fino $^{1 *}$ Inês Carvalho² Mariana Marques ${ }^{1}$ Manuela Cardoso ${ }^{1}$ Raul E. Piña-Aguilar ${ }^{3,4}$ Cynthia C. Morton ${ }^{3-7}$

${ }^{1}$ Department of Human Genetics, National Health Institute Doutor Ricardo Jorge, Lisbon, Portugal;

2Department of Medical Genetics, Central Lisbon Hospital Center (CHLC), Lisbon, Portugal;

${ }^{3}$ Harvard Medical School, Boston, MA, United States;

${ }^{4}$ Departments of Obstetrics and Gynecology, and of ${ }^{5}$ Pathology, Brigham and Women's Hospital, Boston, MA, United States; ${ }^{6}$ Broad Institute of MIT and Harvard, Cambridge, MA, United States; ${ }^{7}$ University of Manchester, Manchester Academic Health Science Center, Manchester, UK

*These authors contributed equally to the work

\section{Corresponding author:}

Dezső David, Ph.D., Department of Human Genetics National Health Institute Doutor Ricardo Jorge Av. Padre Cruz, 1649-016 Lisbon, Portugal. email: dezso.david@insa.min-saude.pt Fax: (+351) 217526410

Telephone: (+351) 217519322 


\section{Contents}

\section{Supplementary Material and Methods}

Ethics statement, karyotyping and CMA

Affymetrix Human Transcriptome Array 2.0 data analysis 5

Large-insert or large-insert jumping genomic sequencing library preparation and sequencing

Bioinformatic analysis of sequencing data 6

Identification of cluster specific split-reads $\quad 7$

The TAD-Gene Content Tool (TAD-GConTool) 11

The CNV-Content Tool (CNV-ConTool) 14

Visualization of high-resolution chromosome conformation capture data 19

\section{Supplementary Results}

Retrospectively analyzed probands 20

DGRC0006 - t(8;14) 20

DRGR0013 - inv(13) 20

DGRC0025 - t(12;17) 20

DRGR0030 - t(1;3) 21

\section{Supplementary Figures}

Supplementary Fig. 1 Evolution of DGRC0025 phenotypic facial features

Supplementary Fig. 2 Evolution of DGRC0030 phenotypic facial features from 3 months to 11 years of age

Supplementary Fig. 3 Nucleotide sequence of der(16) and der(17) breakpoints aligned against the GRCh38 reference human genome

Supplementary Fig. 4 Nucleotide sequence of der(2) and der(19) breakpoints aligned against the GRCh38 reference human genome

Supplementary Fig. 5 Fusion genes and hypothetical transcripts resulting from the $t(16 ; 17)(q 24.3 ; q 21.31) d n$

Supplementary Fig. 6 Chromatin interaction heatmaps of the $\mathrm{t}(2 ; 19)(\mathrm{p} 13.3 ; q 13.11) \mathrm{dn}$ breakpoint regions for GM12878 lymphoblastoid cell lines and IMR90 fibroblasts at different resolutions

Supplementary Fig. 7 Nucleotide sequence of $\operatorname{der}(8)$ and der(14) breakpoints aligned against the GRCh38 reference human genome

Supplementary Fig. 8 Nucleotide sequence of the paracentric chromosome 13 inversion breakpoints aligned against the GRCh38 reference human genome

Supplementary Fig. 9 Nucleotide sequence of der(12) and der(17) breakpoints aligned against the GRCh38 reference human genome

Supplementary Fig. 10 Nucleotide sequence of $\operatorname{der}(1)$ and $\operatorname{der}(3)$ breakpoints aligned against the GRCh38 reference human genome 
Supplementary Fig. 11 Illustration of DGRC0016-specific genomic imbalance identified at $8 q 24.21$ by sequence coverage and read-pair cluster analysis

Supplementary Fig. 12 Nucleotide sequence of genomic imbalance breakpoints at 3p24.1 and 8q24.21 identified in subject DGRC0016

Supplementary Fig. 13 Tissue-specific expression profiles of ANKRD11 and WNT3 disrupted by the 16q24.3 and 17q21.31 breakpoints, respectively

Supplementary Fig. 14 Tissue-specific expression profiles of ATP6V1B1 and CEP89

Supplementary Fig. 15 Differential expression at exon and exon junction levels of $t(16 ; 17)$ disrupted genes

\section{Supplementary Tables}

Supplementary Table 1 Primers used for validation of structural chromosomal abnormalities and CNVs

Supplementary Table 2 Comparison of the proband's clinical features with those of patients with pathogenic SNVs, deletions and intragenic duplications of $A N K R D 11$ and 16q24.3 microdeletions encompassing ANKRD11

Supplementary Table 3 Comparison of the DGRC0025 - $t(12 ; 17)$ proband's clinical features with those of patients with $A N K S 1 B$ haploinsufficiency syndrome

Supplementary Table 4 Comparison of clinical features between the DGRC0030 - $t(1 ; 3)$ proband and those with pathogenic SNVs and 1q41q42 microdeletions either encompassing WDR26 or not encompassing WDR26

Supplementary Table 5 liGS libraries and mapping metrics of analyzed cases

Supplementary Table 6 Summary of interchr chimeric and intrachr inv type structural variants identified at different resolution

Supplementary Table 7 del and dups identified by different methods, cross validations, at different resolutions and frequencies

Supplementary Table 8 Protein coding and non-coding RNA genes localized
within hESC TADs disrupted by DGRC0016 t(16;17) breakpoints and

Supplementary Table 8 Protein coding and non-coding RNA genes localized
within hESC TADs disrupted by DGRC0016 t(16;17) breakpoints and associated phenotypes

Supplementary Table 9 Protein coding and non-coding RNA genes localized within hESC TADs disrupted by DGRC0019 t(2;19) breakpoints and associated phenotypes

Supplementary Table 10 Protein coding and non-coding RNA genes localized within hESC TADs disrupted by DGRC0019 $t(2 ; 19)$ breakpoints with their associated phenotypes and GWAS data from the referred regions

Supplementary Table 11 del and dups identified in DGRC0016 $t(16 ; 17)$ by readpair clustering and $\mathrm{DoC}$ analysis and cross validated using these data and an SVref dataset

Supplementary Table 12 Protein coding and non-coding RNA genes localized within the $836.05 \mathrm{~kb}$ del at $8 \mathrm{q} 24.21$, and GWAS data from the referred region 
Supplementary Table 13 del and dups identified in DGRC0019 t(2;19) by readpair clustering and DoC analysis and cross validated using these data and an SVref dataset

Supplementary Table 14 Cross-validated, probands'-specific del and dup identified in the retrospectively analyzed subjects at both resolutions

Supplementary Table 15 Probands'-specific inv, ins and cx identified in retrospectively analyzed subjects

Supplementary Table 16 Expression of genes within disrupted hESC TADs in LCLs with DGRC0016 $t(16 ; 17)$

Supplementary Table 17 Protein coding and non-coding RNA genes localized within hESC TADs disrupted by DGRC0006 $\mathrm{t}(8 ; 14)$ breakpoints, their associated phenotypes and GWAS data from the referred regions

Supplementary Table 18 Protein coding and non-coding RNA genes localized within hESC TADs disrupted by DGRC0013 inv(13) breakpoints with their associated phenotypes and GWAS data from the referred regions

Supplementary Table 19 Protein coding and non-coding RNA genes localized within hESC TADs disrupted by DGRC0025 $\mathrm{t}(12 ; 17)$ breakpoints with their associated phenotypes and GWAS data from the referred regions

Supplementary Table 20 Protein coding and non-coding RNA genes localized within hESC TADs disrupted by DGRC0030 $t(1 ; 3)$ breakpoints with their associated phenotypes and GWAS data from the referred regions

\section{Supplementary References}

\section{Appendices}

Appendix S1 (.xlsx) Complete TAD-Gene Content table of the 16q24.3/17q21.31 breakpoint region

Appendix S2 (.xlsx) Output tables of the CNV-ConTool 


\section{Supplementary Material and Methods}

\section{Ethics statement, karyotyping and CMA}

This study was approved by the Ethics Committee of the National Institute of Health Doutor Ricardo Jorge and was carried out according to the Principles of the Declaration of Helsinki of the World Medical Association. Samples were obtained after informed consent of the participants or their legal representatives. Secondary use of DNAs was approved by the Partners HealthCare IRB under the Developmental Genome Anatomy Project (DGAP) protocol.

Analysis of genomic DNAs using Affymetrix (now ThermoFisher Scientific (Waltham, MA, USA)) CytoScan $750 \mathrm{~K}$ or CytoScan HD microarrays was performed according to the manufacturer's instructions with the analysis parameters of marker count 15 and size $35 \mathrm{~kb}$.

\section{Affymetrix Human Transcriptome Array 2.0 data analysis}

Affymetrix Human Transcriptome Array 2.0 data analysis was performed using the Transcriptome Analysis Console (TAC -4.0) annotation file HTA2_0.r3.na36.hg19.a1.transcript.csv. Gene level and alternative splicing analyses were performed using the Signal Space Transformation -Robust Multichip Average method for background correction and intensity normalization, according to the user manual.

The array detection limit is at a 2 -fold change. Fold change (in linear space) of exons and their corresponding junctions (splicing index) were obtained based on normalized exons and junction probe set intensities, respectively.

\section{Large-insert or large-insert jumping genomic sequencing library preparation and sequencing}

Large-insert genomic sequencing (liGS) libraries were generated according to the liGS procedures described by Talkowski and coworkers (Talkowski et al. 2011). In brief, 5 $\mu \mathrm{g}$ of fetal DNA was randomly sheared for a target size of $3 \mathrm{~kb}$, end-repaired, ligated with EcoP15I cap adapter, and after gel size selection of DNA fragments, circularized using a biotinylated internal adapter containing 2 nt overhangs. Post-circularization steps included DNase digestion of non-circularized products, EcoP15I digestion, endrepair of digested DNA fragments, and binding through the biotinylated internal adapter of DNA fragments containing short pieces of DNA from both ends of the inserts to streptavidin-coated magnetic beads.

The following steps of $\mathrm{dA}$ tailing, ligation of universal and barcode adapters and PCR amplification with specific primers were carried out on these streptavidin beadbound DNA fragments. Finally, PCR products were separated from the streptavidin bound fragments and an approximately $200 \mathrm{bp}$ amplicon was gel purified. Multiplex paired-end, 25/26-cycle sequencing of the resulting libraries was performed on an Illumina HiSeq 2000. 


\section{Bioinformatic analysis of sequencing data}

Bioinformatic analysis of sequencing data was carried out as previously described by Talkowski et al. (2011) and Collins et al. (2017).

In brief, overall quality of sequencing data was assessed by FastQC v0.11.4 and raw read pairs (R1) and (R2) in reverse-forward (outward-facing) orientation converted to standard forward-reverse (inward-facing) orientation by using Seatk Version 1.0r82.

The converted mate-pair FASTQ files were aligned with Burrows-Wheeler shortread aligner (BWA v0.7.12) to the reference genome GRCh38/hg38.p9. The alignment output SAM file converted to the binary BAM file format using sambamba v0.6.5 ( $\mathrm{Li}$ and Durbin 2009; Tarasov et al. 2015).

In a post-processing step, aligned read duplicates are marked and removed by picard tools v1.119 and sambamba v0.6.5, respectively. Then each read is locally realigned using the 1000 Genomes Project datasets as reference for insertions/deletions (indels) by the indelRealigner of the Genome Analysis Toolkit (gatk v3.8.1). Finally, base quality is recalibrated using GATK v3.8.1 BaseRecalibrator and the resulting alignment BAM file is coordinate and name sorted by sambamba v0.6.5.

Subsequently, the BAM file containing aligned and name sorted read-pairs was converted to SAM and submitted to the in-house python script improperCLAS.py. The script starts by sampling 10,000 proper-pairs to calculate median and standard deviation (SD) of the insert size (IS). Then, it categorizes the improper pairs as (i) interchromosomal (interchr) or chimeric, if the reads of the same pair are mapped in different chromosomes; (ii) inversion (inv) improper-pairs, if both reads of the same pair are mapped in the same orientation; (iii) deletion (del) for inward facing and (iv) duplication (dup) for outward facing read pairs with an IS larger than the median IS+3*IS SD, previously calculated.

Improper pairs were clustered together by mapping position using readPairCluster v0.1.0. (Talkowski et al. 2011), creating a set of clusters for each category: chimeric, inv, del and dup.

Clusters with $\geq 30 \%$ overlap with biased genome regions showing systematic short-read mappability biases were filtered out based on a so-called "blacklist". The bulk of the blacklist includes genomic regions with consistently high sequencing depth, compiled by Layer et al. (2014). In addition, the blacklist includes annotated gaps in the reference genome assembly GRCh38/hg38.p9 as well as annotated centromeric, telomeric genomic regions.

Chimeric and inv clusters were divided (if possible) according to their pair orientation, defining two breakpoint specific sub-clusters. Chimeric sub-clusters with less than three read-pairs were discarded.

The chimeric sub-clusters were interpreted for the identification of translocations and interchr insertions (ins), while inv sub-clusters were interpreted for the identification of inv, intrachromosomal (intrachr) ins and complex (cx) structural variants (SV). According to Collins et al. (2017) cx variants involve two or more different distinct SV signatures or three or more breakpoints.

Simultaneously, depth-of-coverage (DoC) analysis was performed to allow a cross validation of the genomic imbalances. Concisely, proper read-pairs of the analyzed six 
cases and 27 controls, were submitted to cn.MOPS v1.24 R package, with slight modifications. By default, cn.MOPS divides the genome into $1 \mathrm{~kb}$ bins and counts the number of reads per bin; the introduced modification allows cn.MOPS to count all the bins that a proper read-pair insert spans, not only the bins where the reads are mapped. DoC analysis was performed with $1 \mathrm{~kb}, 3 \mathrm{~kb}, 10 \mathrm{~kb}$ and $30 \mathrm{~kb}$ resolutions, allowing the identification of alterations larger than $3 \mathrm{~kb}, 9 \mathrm{~kb}, 30 \mathrm{~kb}$ and $90 \mathrm{~kb}$, respectively. The results of the four different resolutions were combined using bedtools v2.27.1 merge (Quinlan and Hal 2010), and filtered with the blacklist, as described above for the clustering results.

The filtered DoC results and blacklist-filtered del/ tandem duplication (dup) clusters, were cross validated and searched in SV reference dataset (SVref dataset; Collins et al. 2017). Alterations acknowledged in two of the three (cluster, DoC, SVref dataset) were reported. Potentially pathogenic, novel or non-polymorphic $(<1 \%$ frequency on SVref dataset) SV were analyzed in more detail using CNV-ConTool. Statistical significance was verified using CNView Collins et al. (2016).

The expected resolution of our analysis, liGS resolution, is equal to the median IS plus twice the SD, i.e., $4.5 \mathrm{~kb}$. However, as long as read-pair clusters do not overlap low-complexity regions, our clinically oriented pipeline includes all translocations and interch ins, unbalanced SV such as del and tandem dup above $30 \mathrm{~kb}$, and inv, intrachr ins and cx variants above $10 \mathrm{~kb}$.

\section{Identification of cluster specific split-reads}

A sequence read that overlaps a balanced or unbalanced SV breakpoint is considered a split-read. The detection of such reads enables straightforward identification of breakpoint junctions at nucleotide resolution. Currently, for short reads, standard alignment software is unable to perform such task. The use of short reads makes identification of split-reads difficult, because alignment of sequence reads to multiple genomic positions is inversely proportional to their size. Additionally, although theoretically possible, searching for split-reads at genome level would require a large amount of computational resources.

For identification of split-reads, an algorithm was developed assuming that: a) split-reads were not mapped in previous steps; b) a sub-cluster that defines the narrowest breakpoint interval has been identified; and c) the pair of the split-read is mapped within the breakpoint defining cluster and is marked in the SAM file as "unmapped-mate".

To implement the algorithm, a custom python tool - Cluster specific split-read finder - was developed (source code is submitted on GitHub: https://github.com/DGRC-PT/cluster specific split read finder). The script depends on the BWA software for read mapping and on an application programming interface (API) for connection to the NCBI database through biopython v1.68 (Cock et al. 2009), to retrieve FASTA sequences of specific genomic regions.

The algorithm uses as mapping reference the genomic position of the narrowest breakpoint-spanning interval defined by each breakpoint specific sub-cluster, plus 25 bp on each side, or in tandem dup and ins cases $5 \mathrm{~kb}$ regions on each side of the duplicated/inserted region.

Additionally, it requires the reference genome version and the SAM file obtained from the initial mapping. 
The algorithm can be divided in two parts: data selection, where the potential splitreads and breakpoint regions are selected and prepared for analysis; and read processing, where BWA tries to map iteratively the potentially split-reads against the breakpoint regions. An example with a translocation cluster is depicted in Fig. S1.

Data selection consists of:

i) retrieving the FASTA sequences for mapping reference through NCBI API and BWA indexing; and

ii) selection, from the SAM file, of unmapped mate read-pairs localized within the breakpoint defining clusters, for posterior processing.

Read processing includes:

i) alignment of the first and last $5 \mathrm{bp}$, designated as read chunks, of each unmapped read, against the reference sequences, and storage of the mapping data;

ii) repeated realignment of chunks after sequentially increasing their size by 1 bp until no read chunk has a possible alignment or until the chunk size reaches the unmapped mate read size; and

iii) validation of alignment results, outputting only those where read chunks of an unmapped read were mapped to different breakpoint regions, and the sum of the length of the chunks is equal or greater than $80 \%$ length of the unmapped read. To contemplate the possibility of ins in the breakpoint regions, the output also includes reads where only one of the chunks mapped, since that chunk comprises at least $68 \%$ of the read length.

Results are further validated based on the plausibility of their genomic positions, their orientation relative to each other, to the karyotype and to the reciprocal breakpoint. 


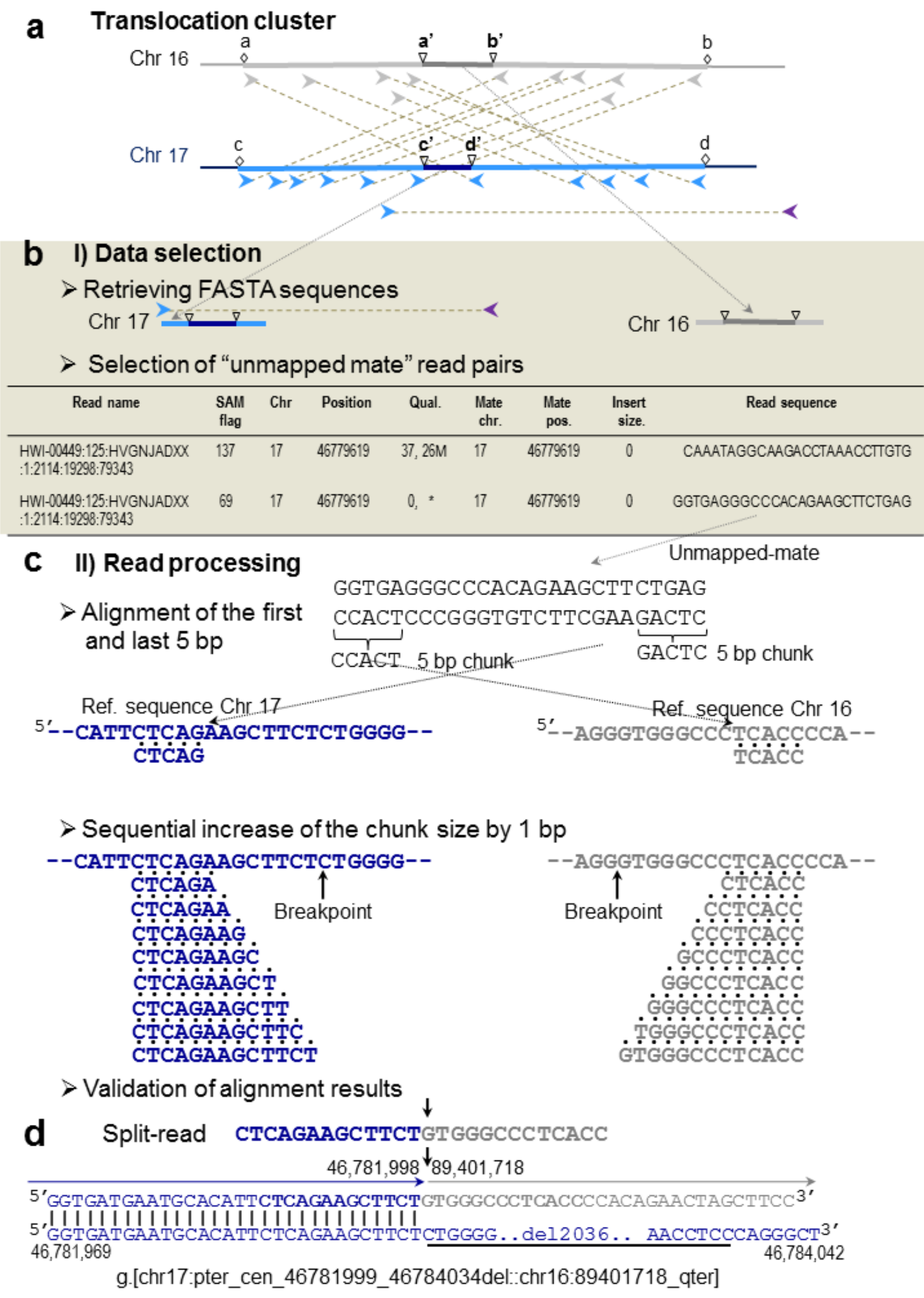

Fig. S1 Identification of split-reads

a Translocation cluster identified by liGS data analysis. Arrowheads depict reads. Reads mapped to chromosome 16 are in gray whereas those on chromosome 17 are 
in blue. Arrowheads linked with dashed line are mate-pair read-pairs. Mate-pair reads in different colors are improper pairs. The unmapped-mate read is colored in violet. Open diamonds indicate the chromosome 16 and 17 translocation breakpoint regions defined between genomic positions $a-b$ and $c-d$, respectively. Triangles indicate the narrowest breakpoint-spanning intervals between a'-b' and c'-d'.

b Data selection step. The retrieved narrowest chromosome 16 and 17 breakpointspanning FASTA sequences are in dark blue and gray, respectively. The additional flanking sequences on each side are in light blue and gray, respectively.

Below are shown lines of the SAM file corresponding to the unmapped-mate read-pair, where the first line corresponds to the mapped read and the second to the unmapped read. SAM flag defines read properties, including mapped and unmapped (Sequence Alignment/Map format specification). As default, BWA transcribes the chromosome and the genomic position of the mapped read for the unmapped-mate. The insert size is zero due to the unmapped read.

c Successive alignment steps of read chunks to the reference FASTA sequences. Arrows indicate the breakpoint positions.

d Nucleotide sequence of the der(17) junction fragment aligned against the identified split-read (also see Supplementary Fig. 3).

The script for the identification of split-reads is executed by the command line:

\% python split_reads_V8.py [chr:a-a'-b'-b] [chr:c-c'-d'-d] [SAM file] [reference genome] [inv/trans/del/dup/ins]

The specific command line used for identification of the $t(16 ; 17)$ split-reads is:

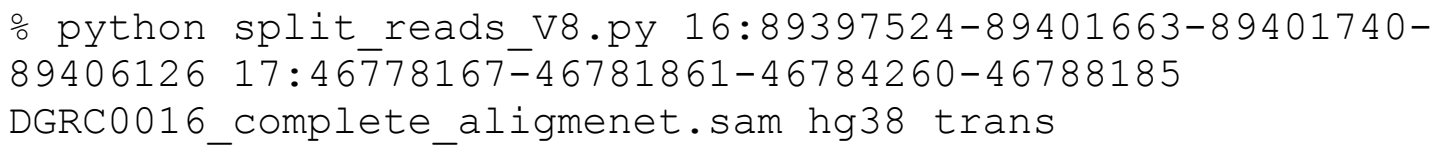

Output results in tab-separated text format from the command-line interface and in table format are shown below (Table S1).

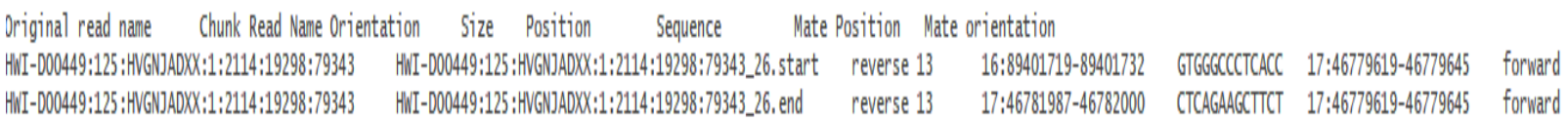

Table S1 Output results of the $t(16 ; 17)$ split-read identification in table format

\begin{tabular}{|c|c|c|c|c|c|c|}
\hline \multirow{2}{*}{ Original read name } & \multirow{2}{*}{ Chunk read name } & \multirow{2}{*}{ Strand } & \multirow{2}{*}{$\begin{array}{l}\text { Chunk } \\
\text { size }\end{array}$} & \multirow{2}{*}{ Alignment position Sequence } & \multicolumn{2}{|c|}{ Mate alignment Mate } \\
\hline & & & & & position & Strand \\
\hline $\begin{array}{l}\text { HWI-D00449:125:HVGNJA } \\
\text { XX:1:2114:19298:79343 }\end{array}$ & $\begin{array}{l}\text { DHWI-D00449:125:HVGNJAD } \\
\text { XX:1:2114:19298:79343.start }\end{array}$ & Reverse & 13 & 16:89401719-89401732 GTGGGCCCTCACC & $\begin{array}{l}17: 46779619- \\
46779645\end{array}$ & Forward \\
\hline $\begin{array}{l}\text { HWI-D00449:125:HVGNJA } \\
\text { XX:1:2114:19298:79343 }\end{array}$ & $\begin{array}{l}\text { DHWI-D00449:125:HVGNJAD } \\
\text { XX:1:2114:19298:79343.end }\end{array}$ & Reverse & 13 & 17:46781987-46782000 CTCAGAAGCTTCT & $\begin{array}{l}17: 46779619- \\
46779645\end{array}$ & Forward \\
\hline
\end{tabular}

The output includes the name of the original read and respective chunks (the chunk name is similar to the read name, with the suffix .start/.end according to the position of the aligned chunk within the unmapped read), the orientation in which the chunk is mapped, the size, position and sequence of the chunk, and information about its previously mapped mate.

Although the der(17) split-read is given as an example, split-reads defining del, dup, inv and ins breakpoints have been identified. 
Four such instances are:

i) in DGRC0016 a $53 \mathrm{~kb}$ del at 3p24.1 shown at Supplementary Fig. 12 (chr3:27,354,65_AAGGAAGGGCAGTTC::chr3:27,408,191_ACTTATCTATA); ii) in DGRC0006 a $16 \mathrm{~kb}$ inv at 4p13 (see Supplementary Table 15) (GCTGC_chr4:43,736,483::chr4:43,751,504_CTCCACTTTCTATACCTTAA); iii) in DGR $\bar{R}$ C0025 a polymorphic $193 \mathrm{~kb}$ dup at $4 \mathrm{q} 13.1$ (chr4:63,872,078_GTACAG::CA::chr4:63,674,807_GAAAAAGAACCCCAAAC); and iv) in DGRC0006 an interchr inverted ins of a $29 \mathrm{~kb}$ fragment from 2q36.3 to $15 \mathrm{q} 26.1$ (chr15:93,296,516_ATAAGAAAAAAAATACG::GCCATGAA_chr2:227,358,389).

\section{The TAD-Gene Content Tool (TAD-GConTool)}

The TAD-GConTool was developed in python with a Common Gateway Interface (CGI) that allows easy, user-friendly applications through any internet browser. The source code of the tool is submitted to GitHub: https://github.com/DGRC-PT/TADGConTool and, can be accessed online at http://dgrctools-insa.min-saude.pt.

Based on genomic positions of rearrangement breakpoints the tool identifies breakpoint Topologically Associated Domains (brTADs) and adjacent TADs (upstream TAD-1 and downstream TAD+1), as well as protein coding and non-coding RNA genes, at exon/intron resolution, disrupted by the breakpoints. Genomic position of TAD boundaries in human embryonic stem cells (hESC) and IMR90 fibroblasts (IMR90) are according to Dixon et al. (2012) whereas in human lymphoblastoid cell line (LCL) GM12878 is according to Moore et al. (2015). Because the referred TAD boundary coordinates are only available in the GRCh37/hg19 genome version, these were converted to the GRCh38/hg38 genome assembly using the UCSC Batch Coordinate Conversion (liftOver) tool.

To run the TAD-GConTool, the necessary input data are shown in Fig. S2:

i) the reference genome assembly;

ii) the reference cell line (different cell types must be analyzed independently);

iii) the additional adjacent TADs to be included in the report table (by default, the three TADs are selected for the complete table whereas only the brTAD is included in the report table);

iv) the type of alteration; and

v) the breakpoint information (chromosome and genomic positions). 


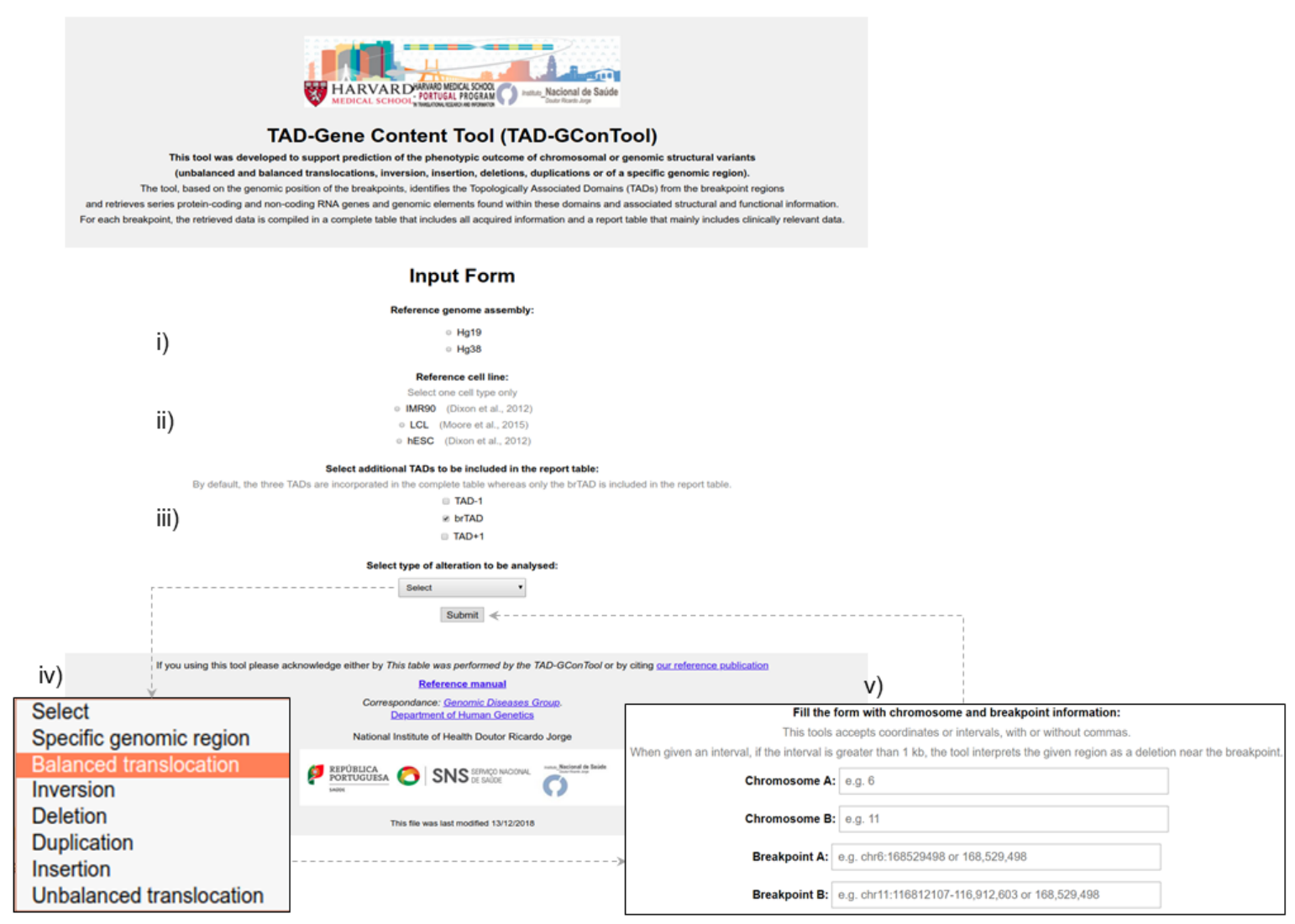

Fig. S2 Landing page of the TAD-Gene Content Tool

The necessary input parameters must be selected as follows:

i) genome version;

ii) reference cell line;

iii) additional adjacent TADs (TAD-1 and TAD+1) to be included in the report table;

iv) within the drop-down list, the alteration type to be analyzed; and

v) data defining the SV (chromosomes and breakpoints information).

Finally, pressing the "Submit" button will launch the tool.

By default, the complete table includes all three TADs (TAD-1, brTAD and TAD+1) whereas the reference table only the brTAD. In this case, unbalanced translocations are those resulting from unequal meiotic segregation of balanced translocations. The tool is unable to handle complex chromosome rearrangements; therefore, these must be subdivided into separate or simpler alterations.

Subsequently, the tool retrieves a series of protein-coding and non-coding RNA genes and genomic elements found within the selected TADs and associated structural and functional information, summarized in Table S2. In addition, the tool retrieves the clinical phenotypes associated with identified genes and highlights those causing major dominant developmental disorders (McKusick 1998; Wright et al. 2015), since these are the most important for prediction of the phenotypic outcome of de novo balanced chromosomal abnormalities.

Data are compiled in two distinct output tables per each breakpoint that can be downloaded through the output page shown in Fig. S3. A complete table (Appendix S1.xlsx) includes all acquired information and a report table (Supplementary Tables 8 and $\underline{9}$ ) mainly includes clinically relevant data. The latter may be included in patient reports. 
Additionally, this tool, based on the genomic position of the breakpoints and affected chromosomes establishes the sequence based nomenclature of the rearrangement according to the International System for Human Cytogenomic Nomenclature 2016 (ISCN 2016) (McGowan-Jordan et al. 2016). The tool will be updated to comply with upcoming revisions to the ISCN.

Table S2 Data retrieved by the TAD-GConTool

\begin{tabular}{|c|c|c|c|}
\hline Data description & Designation & WEB resource & Reference \\
\hline $\begin{array}{l}\text { Protein-coding and non-coding RNA genes } \\
\text { (lincRNA, and miRNA) and associated structural } \\
\text { information (genomic location, exons/introns, } \\
\text { strand, biotype) }\end{array}$ & & $\begin{array}{l}\text { https://www.ensembl } \\
\text {.org }\end{array}$ & $\begin{array}{l}\text { Zerbino et al. } \\
(\underline{2018})\end{array}$ \\
\hline $\begin{array}{l}\text { HGNC gene name and symbol and link to its } \\
\text { integrated GeneCard database }\end{array}$ & GeneCards & $\begin{array}{l}\text { https://www.genecar } \\
\text { ds.org/ }\end{array}$ & $\begin{array}{l}\text { Stelzer et al. } \\
(\underline{\underline{2016}})\end{array}$ \\
\hline $\begin{array}{l}\text { OMIM genes and associated phenotype IDs and } \\
\text { their inheritance }\end{array}$ & OMIM & https://omim.org/ & $\begin{array}{l}\text { McKusick } \\
(\underline{1998)}\end{array}$ \\
\hline $\begin{array}{l}\text { The probability of being haploinsufficiency }(\mathrm{HI}) \\
\text { sensitive expressed as } \mathrm{HI} \text { index }\end{array}$ & DECIPHER & $\begin{array}{l}\text { https://decipher.sang } \\
\text { er.ac.uk/ }\end{array}$ & $\begin{array}{l}\text { Huang et al. } \\
(\underline{2010}) \\
\text { Firth et al. } \\
(\underline{2009)}\end{array}$ \\
\hline $\begin{array}{l}\text { The loss of function (LoF) intolerance, } \\
\text { expressed as a ratio between observed / } \\
\text { expected (oe) number of LoF variants }\end{array}$ & gnomAD & $\begin{array}{l}\text { http://gnomad.broadi } \\
\text { nstitute.org/ }\end{array}$ & $\begin{array}{l}\text { Lek et al. } \\
(\underline{2016)})\end{array}$ \\
\hline $\begin{array}{l}\text { Genetic, genomic, and biological data on the } \\
\text { mouse ortholog }\end{array}$ & MGD-MGI & $\begin{array}{l}\text { http://www.informatic } \\
\text { s.jax.org/ }\end{array}$ & $\begin{array}{l}\text { Bult et al. } \\
(\underline{2008)})\end{array}$ \\
\hline $\begin{array}{l}\text { Clinical phenotypes association based on the } \\
\text { literature. }\end{array}$ & DDG2P & $\begin{array}{l}\text { https://www.ebi.ac.u } \\
\text { k/gene2phenotype }\end{array}$ & $\begin{array}{l}\text { Wright et al. } \\
(\underline{2015})\end{array}$ \\
\hline
\end{tabular}




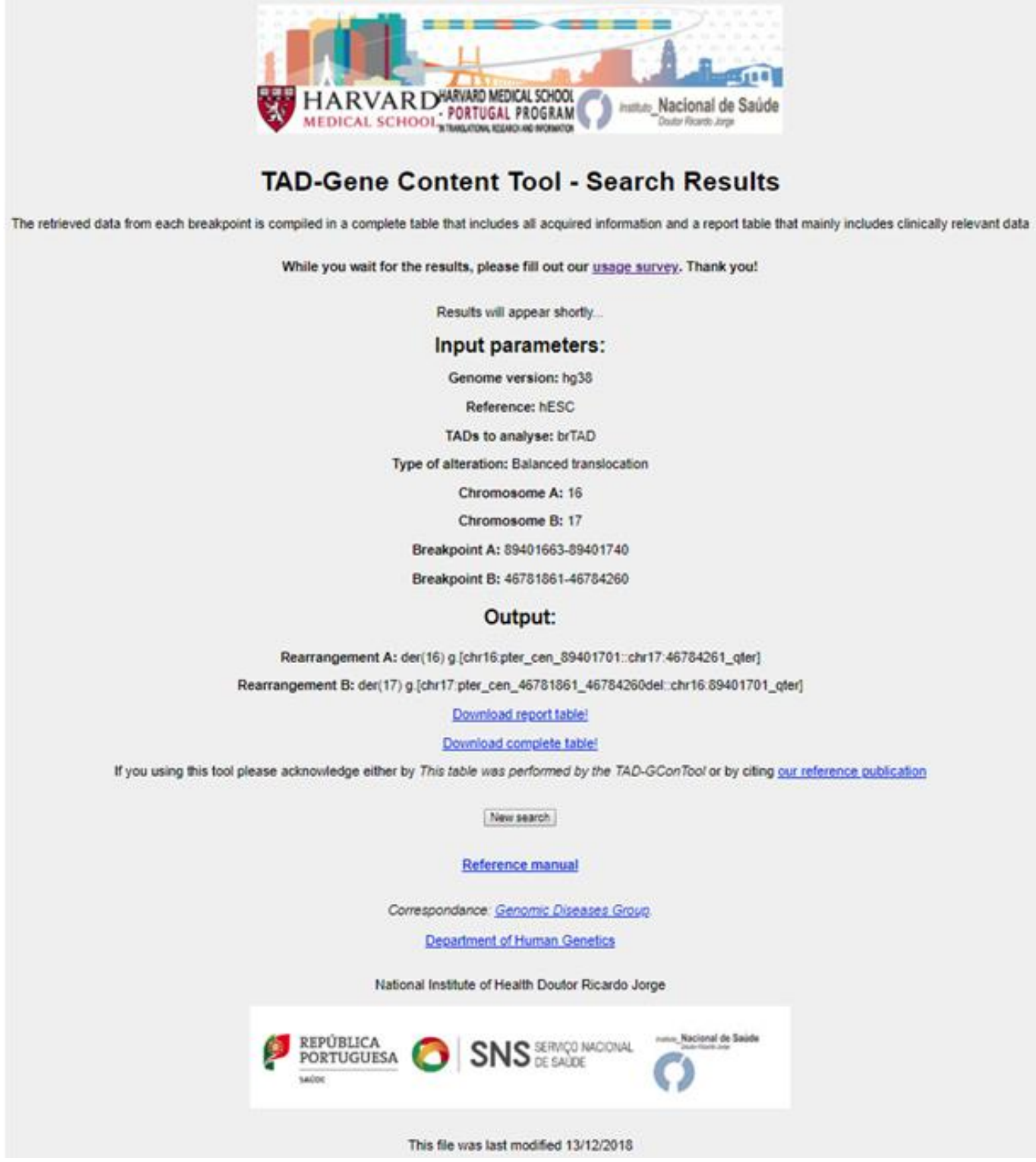

Fig. S3 Search results page of the TAD-Gene Content Tool

The page includes a usage survey, the summary of the input parameters, sequencebased nomenclature of the breakpoints according to ISCN 2016, and links to download the report and complete tables as well as the survey page.

\section{The CNV-Content Tool (CNV-ConTool)}

This tool was essentially developed to allow a faster and more informed evaluation of the SV identified in analyzed subjects. For that, CNV-ConTool is able to define the overlap between patient-specific breakpoints and CNVs and those reported in public databases. The reasoning behind this tool is summarized in Fig. S4. For breakpoint analysis, the tool identifies and reports all overlapping CNVs, whereas for unbalanced alterations, a mutual overlap or a query comprised by the reference search can be applied. Additionally, the tool identifies protein-coding and non-coding RNA genes 
disrupted or deleted by these patient-specific alterations and performs a mutual overlap search.

a

\begin{tabular}{llll}
\multicolumn{1}{c}{ Breakpoint query } \\
del A
\end{tabular}

b Mutual Overlap

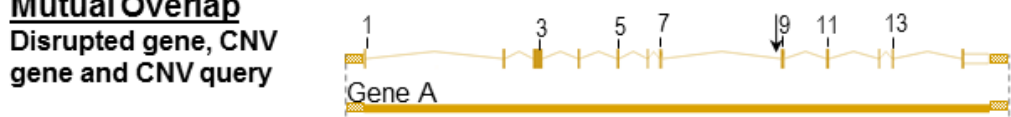

del, dup, inv

\begin{tabular}{|c|c|c|c|c|c|}
\hline & \multicolumn{3}{|c|}{ Reference del C } & & \\
\hline & \multicolumn{3}{|c|}{ Reference dup D } & & \\
\hline \multirow{3}{*}{$\begin{array}{l}\text { Disrupted or } \\
\text { gene A }\end{array}$} & Cutofi & & & & \\
\hline & $80 \%$ & $\begin{array}{l}\text { gene } A \text { vs. reference del } C \\
\text { reference del } C \text { vs. gene } A\end{array}$ & $\begin{array}{l}=100 \% \\
=83 \%\end{array}$ & $\checkmark$ & Included in the output tables \\
\hline & $70 \%$ & $\begin{array}{l}\text { gene } A \text { vs. reference dup } D \\
\text { reference dup } D \text { vs. gene } A\end{array}$ & $\begin{array}{l}=58 \% \\
=76 \%\end{array}$ & $\underset{x}{x}$ & Not included in the output tables \\
\hline \multirow[t]{2}{*}{ del, dup, inv } & $70 \%$ & $\begin{array}{l}\text { query vs. reference del } \mathrm{C} \\
\text { reference del } \mathrm{C} \text { vs. query }\end{array}$ & $\begin{array}{l}=76 \% \\
=76 \%\end{array}$ & & Included in the output tables \\
\hline & $80 \%$ & $\begin{array}{l}\text { query vs. reference del C } \\
\text { reference del C vs. query }\end{array}$ & $\begin{array}{l}=76 \% \\
=76 \%\end{array}$ & $\left.\begin{array}{l}x \\
x\end{array}\right]$ & Not included in the output tables \\
\hline
\end{tabular}

C Query comprised by the reference

CNV query

\begin{tabular}{|c|c|c|c|c|}
\hline \multicolumn{5}{|c|}{ Reference del E } \\
\hline \multicolumn{5}{|c|}{ Reference dup F } \\
\hline \multicolumn{5}{|c|}{ Reference del G } \\
\hline \multirow[b]{2}{*}{ del, dup, inv } & query & $=100 \%$ & $\checkmark$ & Included in the output tables \\
\hline & $\begin{array}{l}\text { query } \\
\text { query }\end{array}$ & $\begin{array}{l}=87 \% \\
=33 \%\end{array}$ & $\begin{array}{l}X \\
X\end{array}$ & Not included in the output tables \\
\hline
\end{tabular}

Fig. S4 Schematization of the mutual overlap and query comprised by the reference approaches and retrieved data

Vertical arrow indicates the breakpoint position. Reference dels are in red whereas dups are in blue. Gray dashed vertical lines outline the overlap between the query and reference alterations.

a Breakpoint genomic position and breakpoint-flanking region. All CNVs overlapping the query genomic position or breakpoint-flanking region are retrieved.

b Mutual overlap approach applied in CNVs (del, dup), inv and affected genes.

Affected genes by either CNVs or breakpoints are depicted in yellow. Additional gene flanking genomic sequences included in the overlap search are depicted by shaded regions flanking the query genes. The query CNV is illustrated by the dashed back bar. In this approach, the percentage of overlap between query vs. reference and reference vs. query is computed, which ensures that, these show approximately the same size, and can be considered as similar. Only alterations with overlaps above the cutoff value (defined by the user), are included in the output tables. 
c Query comprised by the reference search for del, dup and inv. In this approach, only the query vs. reference overlap percentage is taken into consideration: reference CNVs that cover $100 \%$ of the query are included in the output tables. This approach can be applied for CNVs and inv, but not for the affected genes, which by default are analyzed with the mutual overlap approach.

CNV-ConTool was developed in python with a user-friendly CGI interface, similar to TAD-GConTool. The source code of the tool is submitted to GitHub: https://github.com/DGRC-PT/CNV-ConTool and, can be accessed online at http://dgrctools-insa.min-saude.pt.To run the CNV-ConTool, the necessary input parameters must be selected and query data specified as shown in Fig. S5.

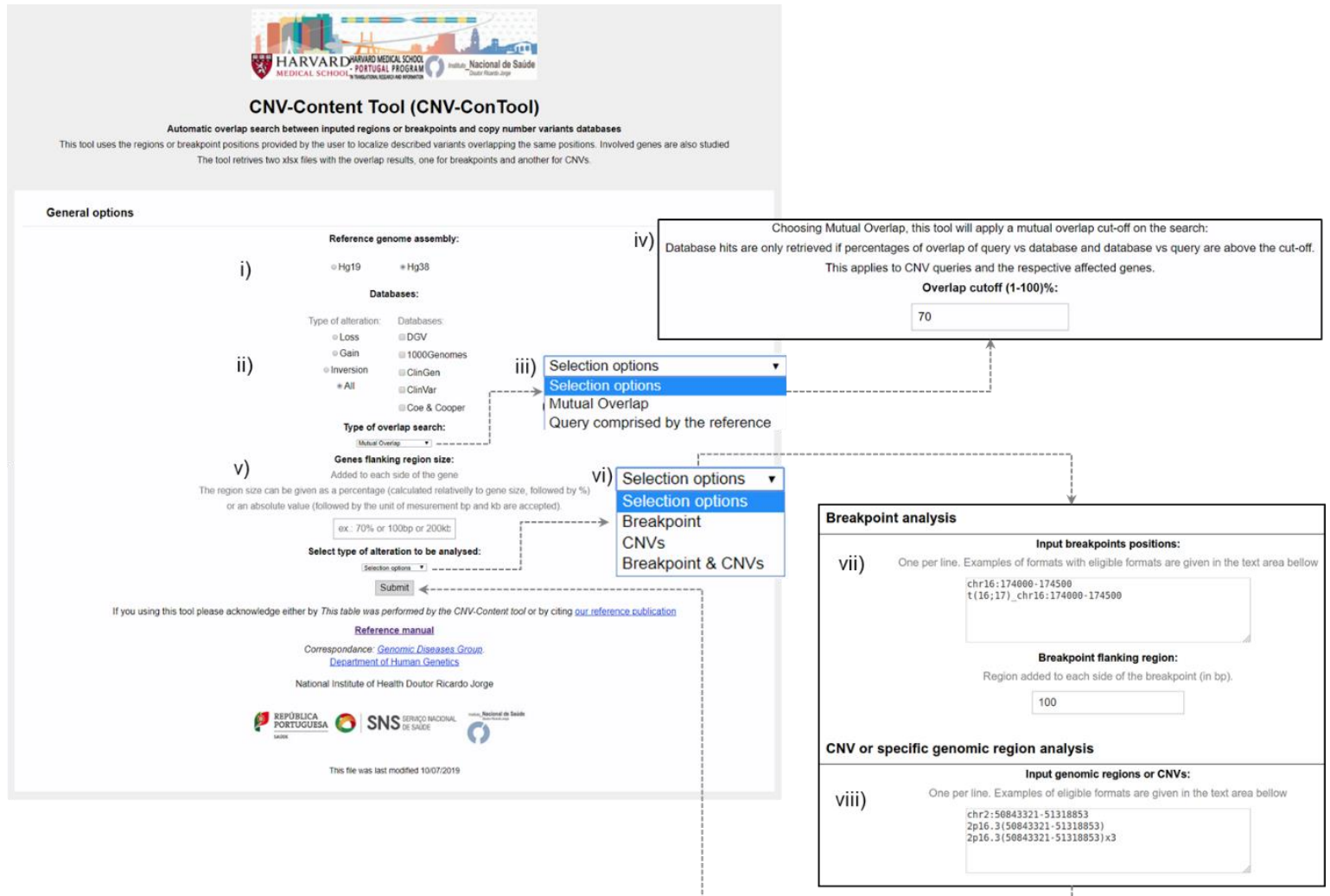

Fig. S5 Landing page of CNV-ConTool with input parameters and query data Input parameters and query regions must be specified as follows:

i) genome version;

ii) type of alteration to search against and reference databases (see Table S3);

iii) type of overlap search: mutual overlap or query comprised by the reference (see Fig. S4).

iv) overlap cutoff, available when mutual overlap is chosen;

v) flanking gene regions to be included in the overlap search (the size of these can be given as an absolute value in bp or $\mathrm{kb}$, or as a relative value, a percentage calculated relative to the size of each gene) - optional;

vi) select the type of analysis (breakpoints, CNVs or both);

vii) breakpoint analysis - input the breakpoint positions and the size of the breakpoint flanking region to be analyzed; definition of a region size to be added to each side of the breakpoint - optional; and

viii) CNV or specific genomic region analysis - input the genomic positions of CNVs or of specific genomic regions to be analyzed. The application starts by identifying coding and non-coding genes within the genomic regions to be analyzed. Then, the genomic positions of these genes are included in the overlap search outlined at Fig. S4. 
Table S3 Databases available on CNV-Content tool

\begin{tabular}{|c|c|c|c|}
\hline Data description & Designation & WEB resource & Reference \\
\hline $\begin{array}{l}\text { Database of Genomic Variants: a } \\
\text { comprehensive database of structural } \\
\text { variation in the human genome of healthy } \\
\text { control samples }\end{array}$ & DGV & $\begin{array}{l}\text { http://dgv.tcag.ca/dgv/app/ } \\
\text { home }\end{array}$ & $\begin{array}{l}\text { MacDonald et al. } \\
(\underline{2014})\end{array}$ \\
\hline $\begin{array}{l}\text { Comprehensive database of common } \\
\text { human genetic variation in over } 2500 \\
\text { subjects from } 26 \text { populations }\end{array}$ & $\begin{array}{l}1000 \text { Genomes } \\
\text { Project }\end{array}$ & $\begin{array}{l}\text { http://www.internationalge } \\
\text { nome.org/data }\end{array}$ & $\begin{array}{l}\text { Auton et al. } \\
(\underline{2015})\end{array}$ \\
\hline $\begin{array}{l}\text { Clinical Genome Resource Database } \\
\text { defines the clinical relevance of genes and } \\
\text { variants. Variant classification: benign, } \\
\text { potentially benign, uncertain, potentially } \\
\text { pathogenic, pathogenic }\end{array}$ & ClinGen & $\begin{array}{l}\text { https://www.clinicalgenom } \\
\text { e.org/ }\end{array}$ & $\begin{array}{l}\text { Miller et al. } \\
(\underline{2010}) \\
\text { Kaminsky et al. } \\
(\underline{2011)}\end{array}$ \\
\hline $\begin{array}{l}\text { ClinVar is a public archive of reported } \\
\text { associations between human variations and } \\
\text { phenotypes. }\end{array}$ & ClinVar & $\begin{array}{l}\text { https://www.ncbi.nlm.nih.g } \\
\text { ov/clinvar/ }\end{array}$ & $\begin{array}{l}\text { Landrum et al. } \\
(\underline{\underline{2016}})\end{array}$ \\
\hline $\begin{array}{l}\text { An expanded CNV morbidity map from } \\
\text { almost } 45,000 \text { children with developmental } \\
\text { delay. }\end{array}$ & $\begin{array}{l}\text { Developmental } \\
\text { Delay (Coe \& } \\
\text { Cooper) }\end{array}$ & $\begin{array}{l}\text { https://www.ncbi.nlm.nih.g } \\
\text { ov/dbvar/studies/nstd100/ } \\
\text { https://www.ncbi.nlm.nih.g } \\
\text { ov/dbvar/studies/nstd54/ }\end{array}$ & $\begin{array}{l}\text { Coe et al. }(\underline{2014}) \\
\text { Cooper et al. } \\
(\underline{2011)}\end{array}$ \\
\hline
\end{tabular}

The retrieved data, compiled in two xlsx files, is made available through the output page shown in Fig. S6. The first file contains the CNVs whereas the second the breakpoint data. Both include protein-coding and non-coding RNA genes affected by these regions. Each of these files are composed by a summary of the overlap results across user-defined databases, followed by one specific table per database, with the most complete information (Appendix S2).

The summary table shows for each query region and affected gene the number of CNV hits, frequencies, databases and the best hits. The following database specific tables consider the same results in a more extensive way, including all hits (one per line), respective ID (with link), size in bp, coverage region, type of alteration, support, bibliographic references and both calculated overlap percentages.

The source code for cluster-specific split-reads finder, TAD-GConTool and CNVConTool is available at https://github.com/DGRC-PT/. 


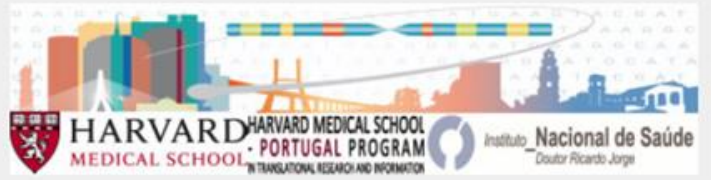

CNV-Content Tool - Search Results

The retrieved data is compiled in two xlsx files, one for breakpoint and another for $\mathrm{CNV}$ results, composed by a summary table, and a set of more complete tables, one for each database used.

While you wait for the results, please fill out our usage survey. Thank you!

Results will appear shortly

Input parameters:

Genome version:hg38

Alteration Data:all

Alteration Database:DGV.1000Genomes, ClinGen, ClinVar,CoeCoop

Type of overlap search: Mutual overlap

Overlap cutoff percentage: $70 \%$

Breakpoint flanking region: 100

Input breakpoint positions:

$t(16 ; 17)$ chr16:89401663-89401740

$t(16: 17)$ chr17:46781861-46784260

Flanquing region size:0bp

Input CNV or genomic region:

$1 \mathrm{p} 31.1(72,300,604-72,346,218) \times 0$

$2 \mathrm{p} 16.3(52,522,407-52,558,349) \times 0$

$3 \mathrm{p} 24.1(27,354,680-27,408,191) \times 1$

6q16.3(103,289,557-103,316,363)×0

$8924.21(129,061,233-129,897,281) \times 1$

16p12.3(19,933,983-19,956,729)x1

Output:

Download CNV tablel

Download breakpoint table

If you using this tool please acknowledge either by This table was performed by the CNV-Content tool or by citing our reference publication

New search

Reference manual

Correspondance: Genomic Diseases Group

Department of Human Genetics

National Institute of Health Doutor Ricardo Jorge

REPUBLICA

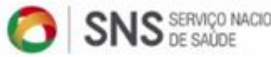

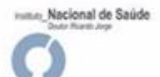

This file was last modified 10/07/2019

Fig. S6 Search results page of the CNV-Content Tool The page includes the summary of the input parameters and regions and links to download the xlsx files. 


\section{Visualization of high-resolution chromosome conformation capture data}

To evaluate genomic interactions, publicly available Hi-C data for IMR90 fibroblasts (Dixon et al. 2012) and GM12878 lymphoblastoid cell lines (Rao et al. 2014) were analyzed.

$\mathrm{Hi}-\mathrm{C}$ interaction data, visualized as heatmaps, of the breakpoint regions from the available cell lines at different resolutions were assessed using Juicebox (Durand et al. 2016), a tool for exploring Hi-C contact map data.

Heatmaps of the selected genomic regions were combined with relevant genomic data:

i) TAD boundaries according to different publications: for hESC (Dixon et al. 2012), IMR90 fibroblasts (Dixon et al. 2012; Rao et al. 2014), and LCL GM12878 (Moore et al. 2015; Rao et al. 2014).

ii) chromatin loops for IMR90 fibroblasts and GM12878 lymphoblastoid cell lines (Rao et al. 2014);

iii) CCCTC-binding factor (CTCF) sites track (IMR90 CTCF IgG-rab ChIP-seq Signal from ENCODE/SYDH) plot;

iv) transcribed human enhancers color-coded according to their specific tissue/cell expression; and

v) the gene map with haploinsufficiency index, and with the LoF intolerance expressed as observed vs. expected (oe) number of LoF variants stated below the genes. 


\section{Supplementary Results}

\section{Retrospectively analyzed probands}

\section{DGRC0006 - t(8;14)}

Proband DGRC0006 is a 12 year-old female with a de novo balanced reciprocal chromosomal translocation between the long arms of chromosomes 8 and 14, $46, X X, t(8 ; 14)(q 11.23 ; q 24.3) d n$ identified during conventional prenatal diagnosis (PND) performed due to advanced maternal age and a previous child with psychomotor developmental delay.

At age 12 years abnormal clinical findings include moderate global developmental delay (HP:0011343) with speech problems as well as feeding difficulties (HP:0011968) and failure to thrive (HP:0001508) with decreased body weight $(\mathrm{HP}: 0004325)\left(<3^{\text {rd }}\right.$ percentile) and height at $15^{\text {th }}$ percentile. Premature pubarche (HP:0012411) with pubic hair growth was present from seven years of age.

Mapping of the breakpoints by liGS followed by Sanger sequencing identified the $8 q 11.23$ breakpoint

at g.[chr8:pter_cen_64,209,134::chr14:83,126,596_83,126,598dup_qter] within IVS 1 of LINC01414 whereas the 14q24.3 breakpoint was identified at g.[chr14:pter_cen_83,126,598::chr8:64,209,135_qter] (Supplementary Fig. 7).

\section{DGRC0013 - inv(13)}

Proband DGRC0013 is a 13 year-old male with a de novo paracentric chromosomal 13 inversion 46, $\mathrm{XY}, \operatorname{inv}(13)(\mathrm{q} 12.3 \mathrm{q} 22) \mathrm{dn}$ identified during conventional PND performed due to advanced maternal age.

Abnormal clinical findings include moderate global developmental delay (HP:0011343), especially at motor level, mild hypotonia (infantile muscular hypotonia, HP:0008947) and generalized joint laxity (HP:0002761).

Cardiac evaluation revealed dilated left ventricle, non-hypertrophied walls, with overall conservation of good systolic function and tricuspid valve ring thickening (abnormality of the tricuspid valve, HP:0001702).

Recurrent upper tract respiratory infections (HP:0002788) that led to amygdalectomy and adenoidectomy. Bilateral cryptorchidism (HP:0008689) and phimosis (HP:0001741) were surgically corrected by orchidopexy and preputioplasty, respectively.

Mapping of the breakpoints by liGS fallowed by Sanger sequencing identified the proximal 13q12.3 inversion breakpoint at position g.28,489,796 within IVS 1 of $F L T 1$ whereas the distal 13q22 breakpoint is at position g.74,831,804 (chr13:g.[ptercen_28,489,793_28,489,795del;28,489,796_74,831,804inv; 74,831,805_74,831,817 del_qter]) (Supplementary Fig. 8).

\section{DGRC0025 - $t(12 ; 17)$}

Proband DGRC0025 is an 8 year-old male with a de novo balanced reciprocal chromosomal translocation between the long arms of chromosomes 12 and 17 $46, X Y, t(12 ; 17)(q 23 ; q 22) d n$ identified during conventional PND performed due to 
increased risk for aneuploidy following $1^{\text {st }}$ trimester combined tests and increased nuchal translucency.

In the neonatal period, pulmonary artery stenosis (HP:0004415) and patent foramen ovale (HP:0001655) were identified by electrocardiography.

Physical examination did not reveal significant dysmorphism other than one glabellar (HP:0001076) and two lumbar hemangiomas (Supplementary Fig. 1). Additional facial features observed in this proband are thin upper lip vermilion, depressed nasal bridge, slightly anteverted nares noted throughout development, and synophrys (HP:0000664) evident from eight years of age (Supplementary Fig. 1 and Supplementary Table 3).

Neurological assessment revealed delayed speech and language development HP:0000750, expressive language delay HP:0002474, and borderline fine motor skills and practical performance. At 48 months, his developmental age was equivalent on average to 39.5 months. Presently he has early intervention support with speech therapy.

Mapping of the breakpoints by liGS followed by Sanger sequencing identified the $12 q 23$ breakpoint g.[chr12:pter_cen_99,637,772_99,637,782del::chr17:51,565,697_qter] within IVS 9 of ANKS1B whereas the 17q22 breakpoint was identified at g.[chr17:pter_cen_51,565,696::chr12:99,637,783_qter] (Supplementary Fig. 9).

Clinical features of DGRC0025 overlap those of individuals with monogenic heterozygous microdeletions in $A N K S 1 B$, reported by Carbonell et al. (2019). Furthermore, we confirm that disruption of this gene results in $A N K S 1 B$ haploinsufficiency syndrome.

\section{DRGR0030 - t(1;3)}

Proband DGRC0030, the third child of a non-consanguineous couple, is a 13 year-old male. Conventional PND performed due to advanced maternal age identified a de novo balanced reciprocal chromosomal translocation between the short arm of chromosome 1 and long arm of chromosome 3, 46,XY,t(1;3)(q42;p25)dn.

The proband's father and siblings are healthy, whereas his mother suffers from bilateral congenital cataracts (HP:0000519) and severe myopia (HP:0011003).

Delivery occurred at 37 weeks gestation and was uneventful. At birth, weight and height were $2.686 \mathrm{~kg}\left(7.5^{\text {th }}\right.$ percentile $)$ and $49.5 \mathrm{~cm}\left(42^{\text {nd }}\right.$ percentile $)$ according to WHO tables, respectively. Head circumference measured $33 \mathrm{~cm}\left(12^{\text {th }}\right.$ percentile) on WHO tables

The proband is characterized by dysmorphic facial features (Supplementary Fig. 2 and Supplementary Table 4) that include coarse face (HP:0000280) with prominent maxilla (HP:0430028) and upper lip (HP:0000215), wide mouth (HP:0000154), widely spaced teeth (HP:0000687), flat nasal bridge (HP:0005280) and a broad full nasal tip (HP:0000455) with anteverted nares (HP:0000463). His growth was stable with weight constantly around the $50^{\text {th }}$ percentile and height around the $25^{\text {th }}$ percentile. The head circumference was always around the $10^{\text {th }}$ percentile.

Cardiovascular examination revealed a complex cardiopathy characterized by tetralogy of Fallot (HP:0001636) with a right aortic arch (HP:0012020) and subaortic interventricular communication. 
Neurologic abnormalities include severe global developmental delay (HP:0011344) with absent speech (HP:0001344) and a severe behavioral clinical condition with hyperactivity (HP:0000752). Multiple stereotypies (HP:0000733), broadbased ataxic gait (HP:0002136), and an aberrant posture with tendency to hyperextension of the neck were also reported. A slight thoracic scoliosis (HP:0002943) developed with time.

Most likely due to severe developmental delay, the proband presented in addition to hypotonia and severe feeding difficulties (HP:0011968), with nocturnal and diurnal enuresis (HP:0000805) and encopresis (HP:0040183).

Additionally, several other congenital anomalies were noted such as bilateral talipes equinovarus (HP:0001776), supernumerary nipples (HP0002558), phimosis (HP:0001741), divergent strabismus (HP:0000486), joint hypermobility (HP:0001382), especially of the interphalangeal joints (HP:0005620). Unilateral hydrocele testis (HP:0000034) and an umbilical hernia (HP:0001537) were diagnosed later in infancy.

Mapping of the breakpoints by liGS fallowed by Sanger sequencing identified the 1q42.11 breakpoint g.[chr1:pter_cen_224398162_224398174del::chr3:10670892_pter] within WDR26 exon 12 whereas the 3p25.3 breakpoint was identified at g.[chr1:qter_224398174::chr3:10670893_10670894del_cen_qter] within IVS 1 of ATP2B2 (Supplementary Fig. 10). WDR26 encodes a WDR domain-containing protein presumably involved in multiple disease-associated signaling pathways.

In conclusion, disruption of WDR26 by the 1q42.11 breakpoint most likely leads to its haploinsufficiency due to nonsense mediated RNA decay, resulting in a complex clinical phenotype matching both Skraban-Deardorff syndrome (SKDEAS OMIM \#617616) (Skraban et al. 2017) and the 1q41q42 microdeletion syndrome. Therefore, DGRC0030 was diagnosed as having severe Skraban-Deardorff syndrome (SKDEAS OMIM \#617616).

Moreover, the proband's clinical features basically confirm the phenotypic overlaps between SKDEAS and the 1q41q42 microdeletion syndrome and affirm the causative role of WDR26 in these phenocopy syndromes. 


\section{Supplementary Figures}

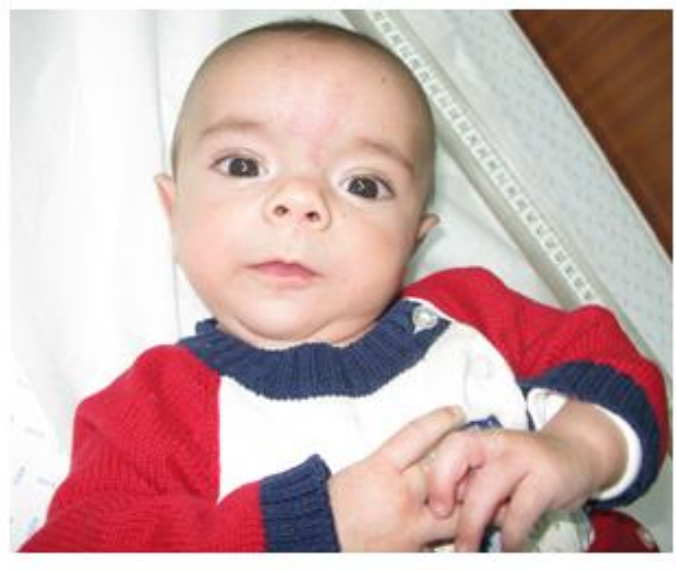

4 months

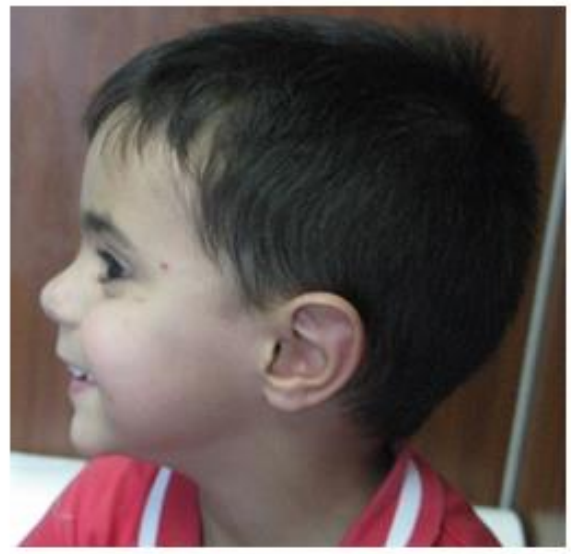

4 years

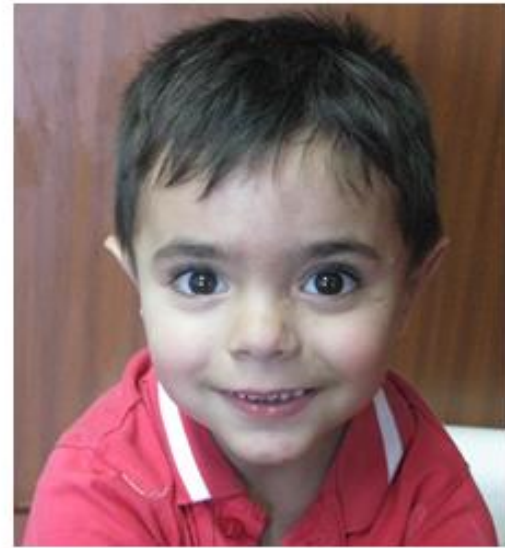

4 years

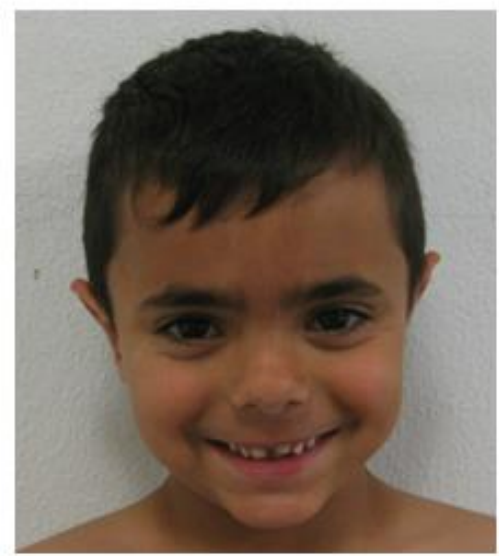

8 years

Supplementary Fig. 1 Evolution of DGRC0025 phenotypic facial features. Glabellar hemangioma, thin upper lip vermilion, depressed nasal bridge and slightly anteverted nares can be seen throughout time. Synophrys became evident from age 8 . 


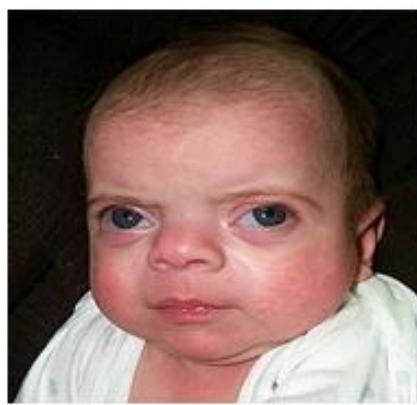

\section{3 months}

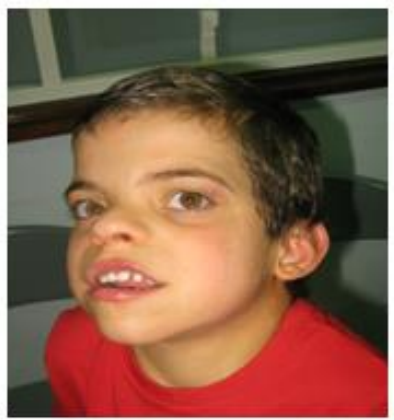

8 years

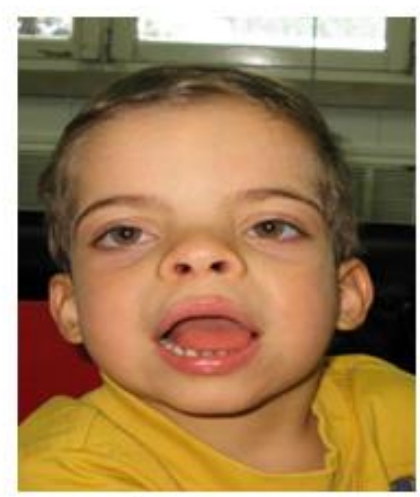

4 years

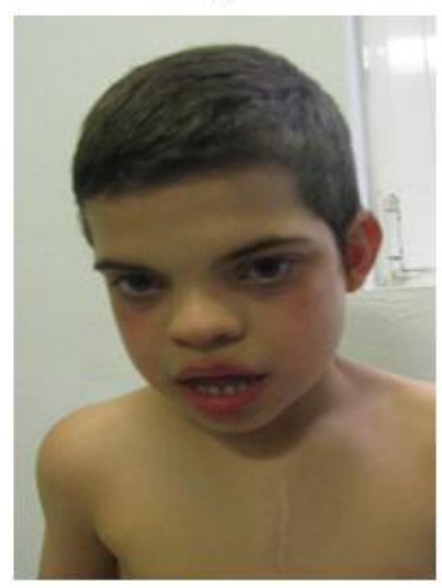

11 years

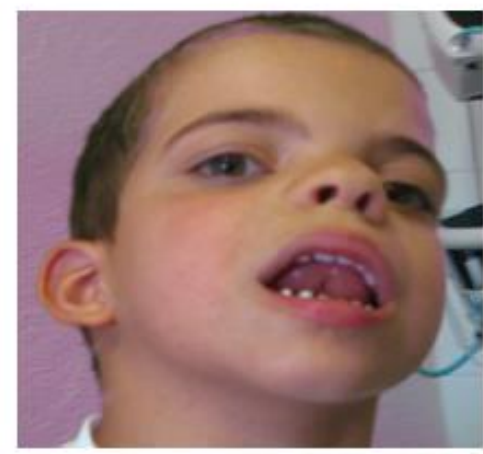

6 years

Supplementary Fig. 2 Evolution of DGRC0030 phenotypic facial features from 3 months to 11 years of age. Coarse facial features, full/broad nasal tip, depressed nasal bridge and anteverted nares are evident throughout time. Tented, protruding upper lip and wide spaced teeth can be seen beginning at 4 years of age. 


\section{DGRC0016}

a $\operatorname{der}(16)$

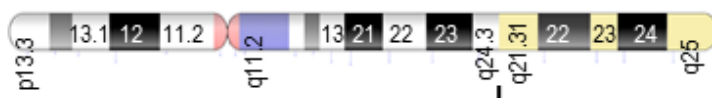

당

СTTTGCAGATGATCCAGGTGAGATCGCTACCAGGGCTCAGCTCTCATTGTGCAGCTGGG

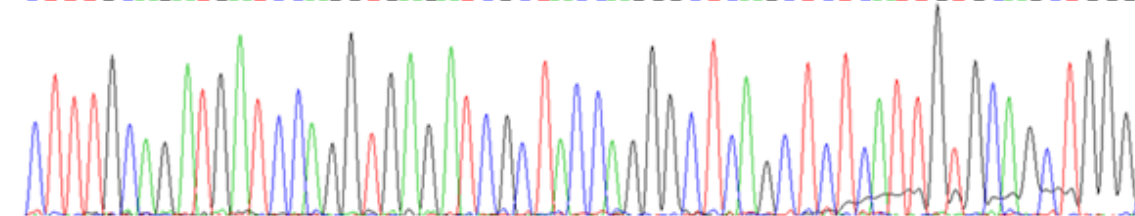

$\stackrel{89,401,715}{\longrightarrow} 46,784,035$

$5^{\prime}$ СTTTGCAGATGATCCAGGTGAGATCGCTACCAGGGCTCAGCTCTCATTGTGCAGCTGGG ${ }^{3}$

${ }^{5}$ CTTTGCAGATGATCCAGGTGAGATCGCTAGGGTGGCCCTCACCCCACAGAACTAGCTT ${ }^{3}$

$89,401,687$

g.[chr16:pter_cen_89401716_89401717del:.:chr17:46784035_qter]

b

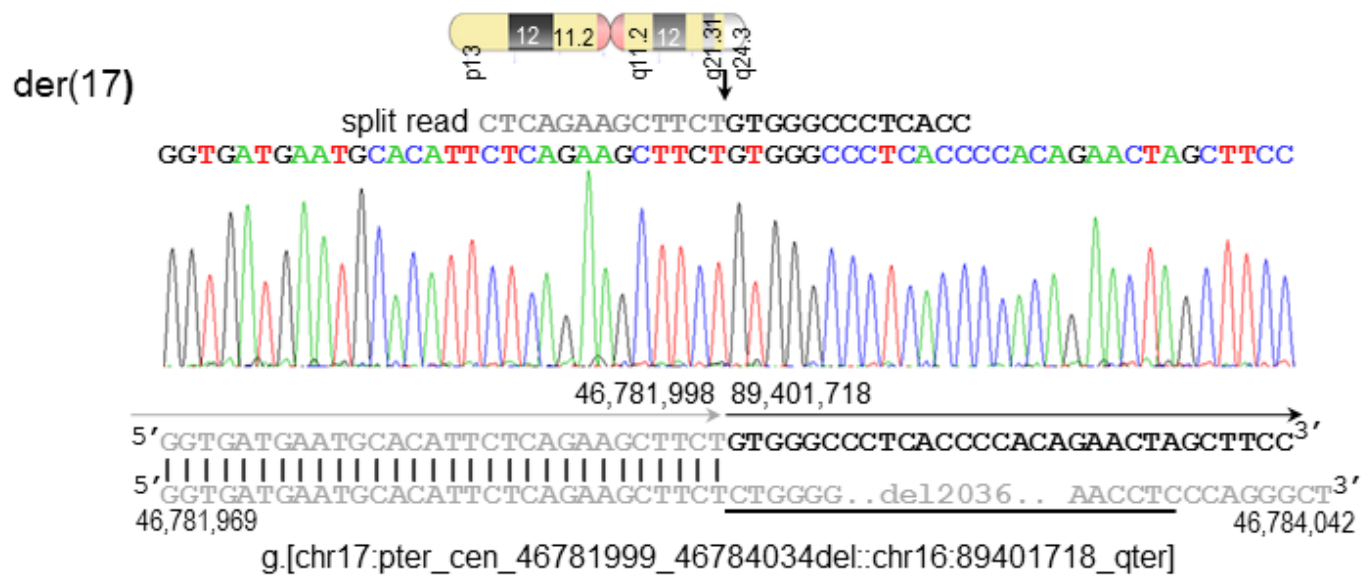

Supplementary Fig. 3 Nucleotide sequence of $\operatorname{der}(16)$ and $\operatorname{der}(17)$ breakpoints aligned against the GRCh38 reference human genome

a Chromosome 16q24.3 breakpoint. The chromosome 16 sequence is in black, whereas the chromosome 17 sequence is in gray. Vertical lines indicate identical nucleotides between derivative and reference chromosomes. The GG dinucleotide deleted from the der(16) breakpoint is underlined.

b Chromosome 17q21.31 breakpoint. The chromosome 16 sequence is in black, whereas the chromosome 17 sequence is in gray. Vertical lines indicate identical nucleotides between derivative and reference chromosomes.

The 2,036 bp del (g.46781999_46784034del) identified at the der(17) breakpoint is underlined. Therefore, the translocation is classified as unbalanced. The translocation is revised and described as seq[GRCh38] 46,XX,t(16;17)(16pter $\rightarrow 16 q 24.3:: 17 q 21.31 \rightarrow 17 q$ ter; 17 pter $\rightarrow 17 q 21.31:: 16 q 24.3 \rightarrow 16$ qter)dn. According to next-gen cytogenetics nomenclature (Ordulu et al. 2014), the translocation is described as 46,XX,t(16;17)(q24;q21.3)dn.seq[GRCh38] $\mathrm{t}(16 ; 17)(16 \mathrm{pter} \rightarrow 16 \mathrm{q} 24.3(89,401,715):: 17 \mathrm{q} 21.31(46,784,035) \rightarrow 17 q$ ter; $17 \mathrm{pter} \rightarrow 17 \mathrm{p} 2$ 1.31(46,781,998::16q24.3(89,401,718) $\rightarrow 16 q$ ter $)$ dn .

NCBI-GenBank accession numbers of the $\operatorname{der}(16)$ and $\operatorname{der}(17)$ junction fragment sequences are MH843735 and MH843736, respectively. 


\section{DGRC0019}

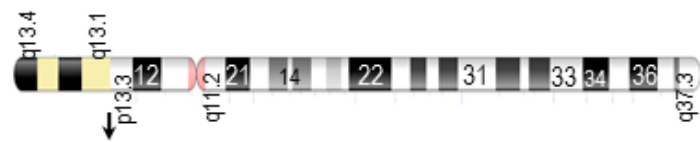

a $\operatorname{der}(2)$

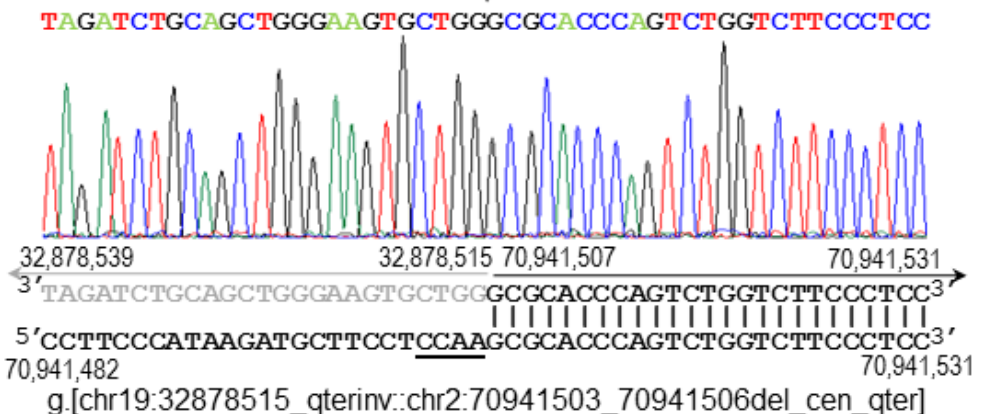

b $\operatorname{der}(19)$

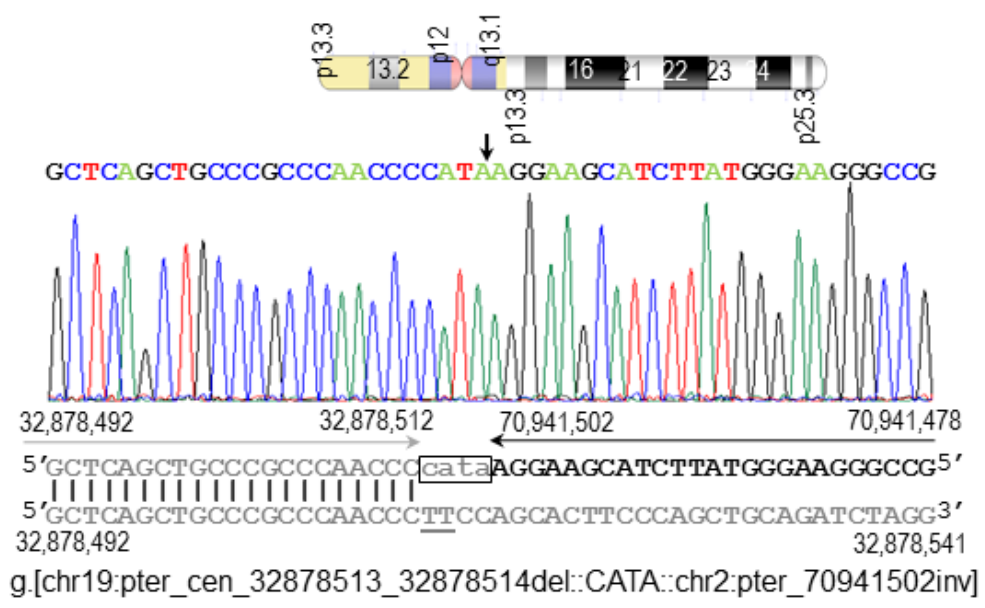

Supplementary Fig. 4 Nucleotide sequence of der(2) and der(19) breakpoints aligned against the GRCh38 reference human genome

a Chromosome 2p13.3 breakpoint. The chromosome 2 sequence is in black, whereas the chromosome 19 sequence is in gray. Vertical lines indicate identical nucleotides between derivative and reference chromosomes. The deleted tetranucleotide CCAA sequence from the der(2) breakpoint is underlined.

b Chromosome 19q13.11 breakpoint. The chromosome 2 sequence is in black, whereas the chromosome 19 sequence is in gray. Vertical lines indicate identical nucleotides between derivative and reference chromosomes. The deleted TT dinucleotide from the $\operatorname{der}(19)$ breakpoint is underlined, whereas the inserted CATA tetranucleotide sequence is boxed and in lowercase.

The translocation is revised and described as seq[GRCh38] $46, X Y, t(2 ; 19)(19 q t e r \rightarrow 19 q 13.11:: 2 p 13.3 \rightarrow 2 q$ ter; $19 p t e r \rightarrow 19 q 13.11:: 2 p 13.3 \rightarrow 2 p t e r) d$ n.

According to next-gen cytogenetics nomenclature (Ordulu et al. 2014), the translocation can be defined as 46,XY,t(2;19)(p13;q13.11)dn.seq[GRCh38] $\mathrm{t}(2 ; 19)$ (19qter()$\rightarrow 19 q 13.11(32,878,515):: 2 p 13.3(+)(70,941,507) \rightarrow 2 q t e r ; 19 p t e r \rightarrow 19 q 13.11(+)(32,87$ 8,512)::CATA::2p13.3(-)(70,941,502) $\rightarrow 2$ pter $)$ dn.

NCBI-GenBank accession numbers of the $\operatorname{der}(2)$ and $\operatorname{der}(19)$ junction fragment sequences are MH843737 and MH843738, respectively. 


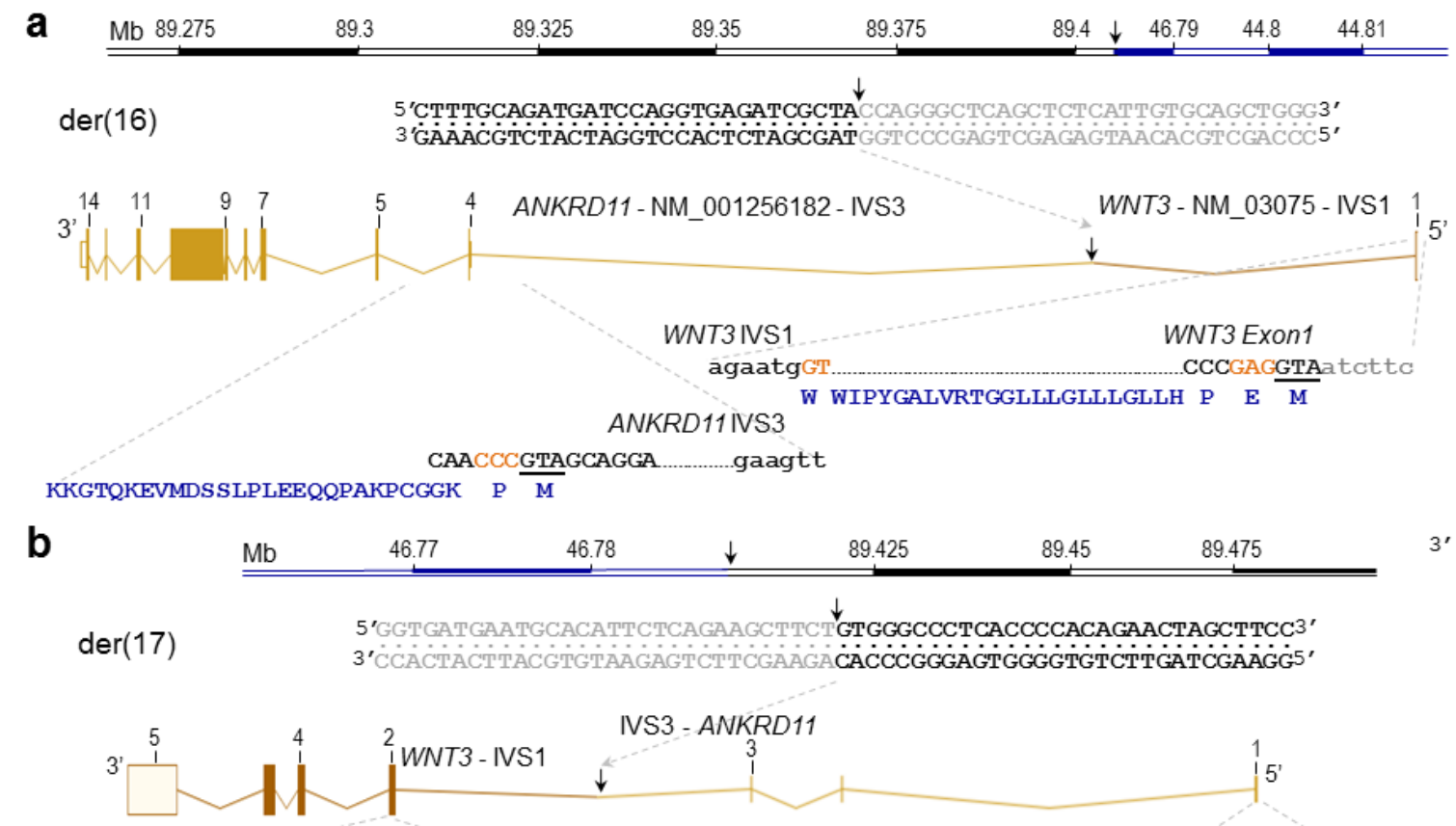

GACCGGGTCCCGGTCCTGgacgtccgtetgtc GGCGCCGGogcaaaggg

Supplementary Fig. 5 Fusion genes and hypothetical transcripts resulting from the $\mathrm{t}(16 ; 17)(\mathrm{q} 24.3 ; \mathrm{q} 21.31) \mathrm{dn}$

a Diagram depicting the fusion gene at der(16) between WNT3 IVS1 and ANKRD11 IVS3. At the top, the physical map across the der(16) breakpoint region with the corresponding junction sequence is shown underneath (chromosome 16 sequence is in black, whereas the chromosome 17 sequence is in gray). Below, schematic representation of the fusion gene between WNT3 exon 1 and ANKRD11 exons 4-14 is shown. Theoretically, WNT3 exon 1 may code for a truncated 26 amino acid polypeptide, while the translational initiation codon within exon 4 of $A N K R D 11$ is intact. Hypothetical translation products are indicated in blue.

b Diagram depicting the fusion gene at der(17) between ANKRD11 IVS3 and WNT3 IVS1. At the top, the physical map across the der(17) breakpoint region with the corresponding junction sequence is shown underneath. Below, schematic representation of the fusion gene between ANKRD11 exons 1-3 and WNT3 exons 25 is shown. The ANKRD11 non-coding exons 1-3 are followed by coding exon 2 of WNT3.

Breakpoint positions are indicated by vertical arrows. Exons are numbered. Exonic and intronic sequences are in upper and lower-case letters, respectively. The 5' untranslated sequences are in lower case gray letters. Coding triplets are highlighted in brown and black and the translational initiation codons (ATG) are underlined. 


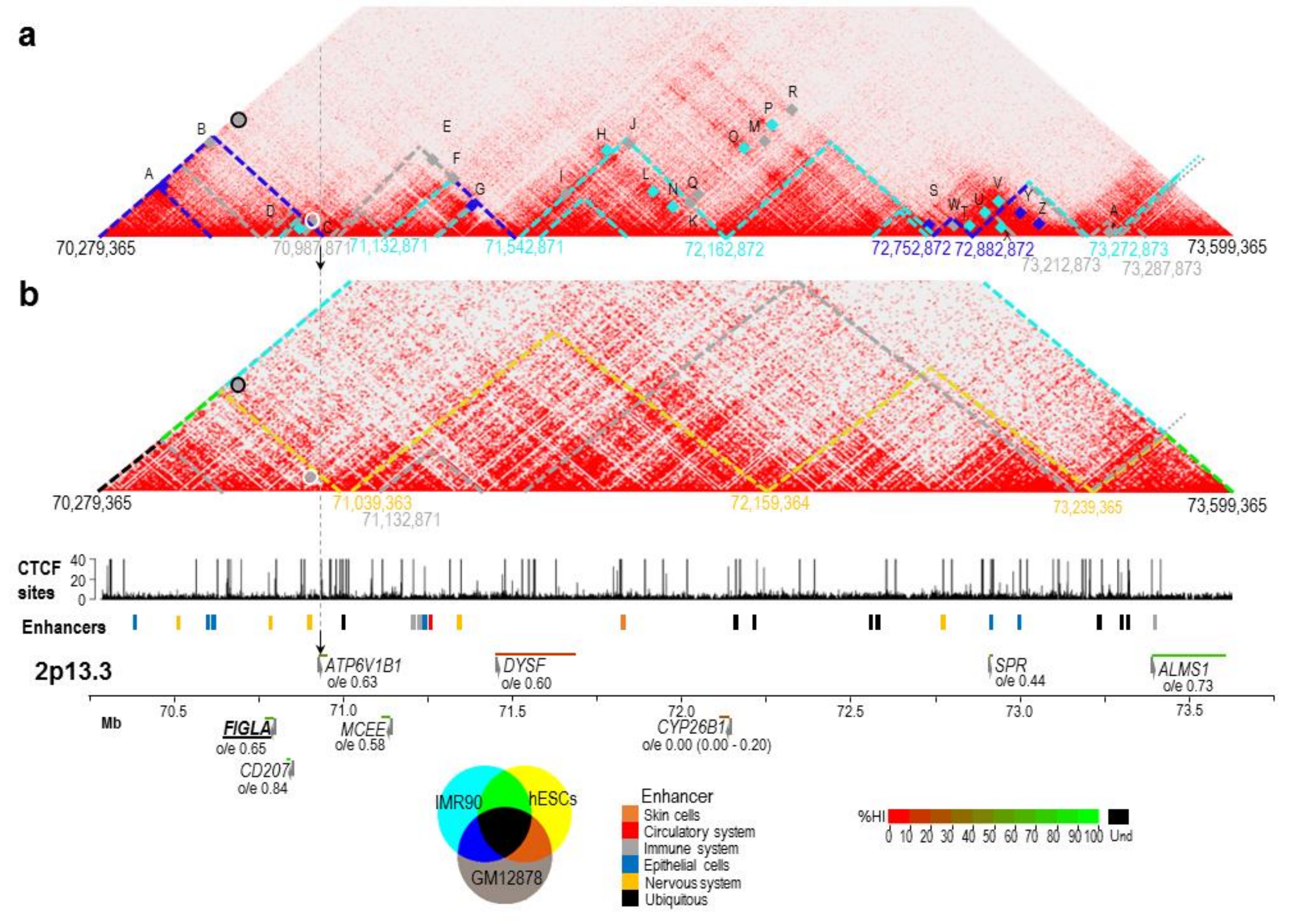



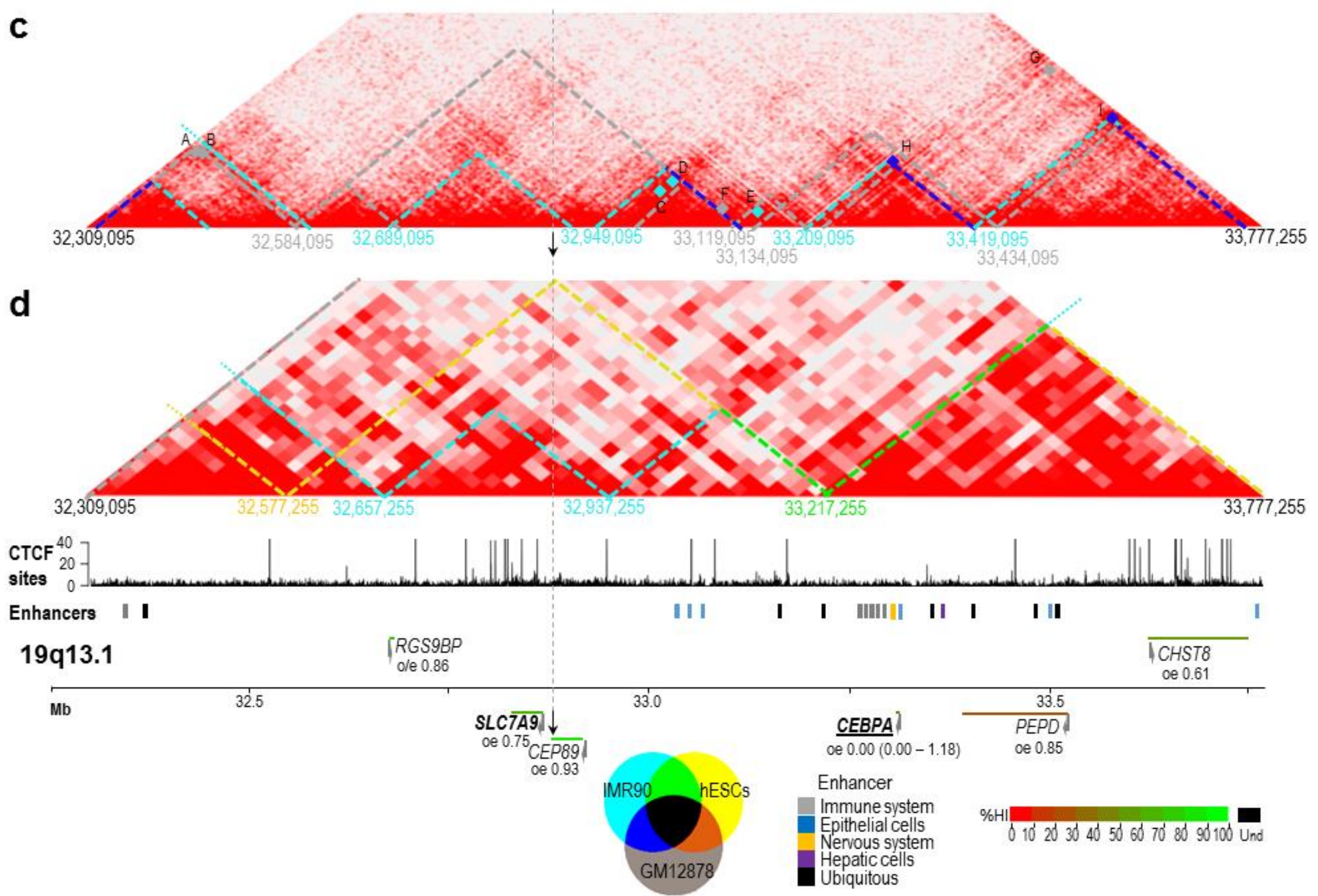
Supplementary Fig. 6 Chromatin interaction heatmaps of the $t(2 ; 19)(p 13.3 ; q 13.11) d n$ breakpoint regions for GM12878 lymphoblastoid cell lines and IMR90 fibroblasts, at different resolutions

a, c Interaction data from GM12878 lymphoblastoid cell lines (LCLs) at $5 \mathrm{~kb}$ resolution from Rao et al. (2014).

b, d Interaction data at $25 \mathrm{~kb}$ resolution from IMR90 fibroblasts from Dixon et al. (2012).

TADs and boundaries for GM12878 and IMR90 are indicated according to Rao et al. (2014). The dashed lines overlaid on the heatmaps are color-coded according to cell line and overlap between these lines. Chromatin loops are depicted by overlaid squares, color-coded by cell line and identified by letters. Loops circled in black and white are disrupted by the breakpoint (Rao et al. 2014). The one circled in black is between two $10 \mathrm{~kb}$ bins, at chr2:70,292,868-70302868, encompassing FAM136A and the promoter-exon 1 region of SNRPG, and, at chr2:71,062,870-71,072,870, encompassing the promoter - exons 1-5 region of NAGK. The second loop, circled in white, is between two $10 \mathrm{~kb}$ bins localized at chr2:70,862,870-70,872,870 and chr2:70,942,870-70,952,870, encompassing exon 2 of ATP6V1B1.None of these genes shows LoF sensitivity.

The black arrows indicate the breakpoint positions. Below is a track depicting CCCTC-binding factor (CTCF) sites from the analyzed region according to the Chip-seq track in IMR90 fibroblasts CTCF IgG-rab ChIP-seq Signal from ENCODE/SYDH). Further below are transcribed human enhancers from the reference genomic region, color-coded according to their tissue/cell specific expression. Beneath the enhancers is shown the gene map across the analyzed genomic region. Forward and reverse arrowheads indicate the position of genes in sense and antisense orientation. Genes are color-coded according to their HI scores. Genes presently associated with autosomal dominant disorders are underlined and bolded, whereas genes associated with both autosomal recessive and dominant disorders are only bolded. The LoF intolerance, expressed as observed / expected (oe) number of LoF variants, are stated below the genes. 


\section{DGRC0006}

a
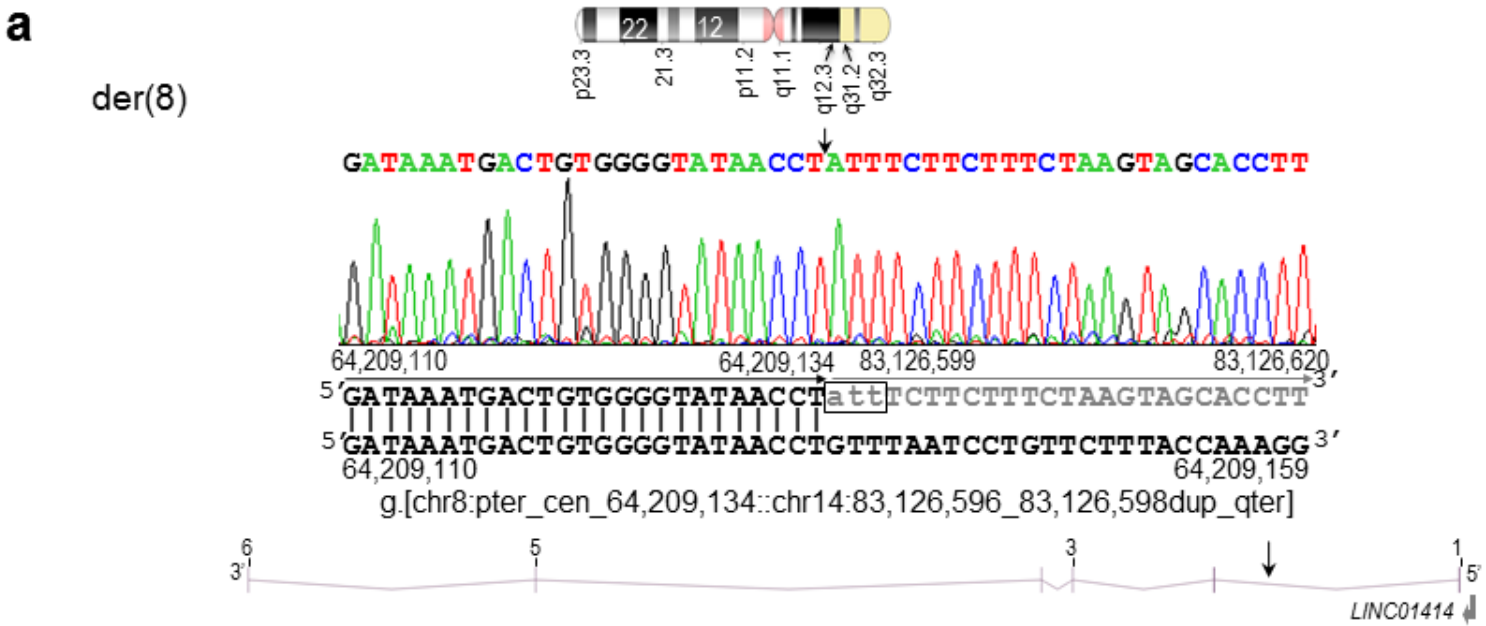

b

$\operatorname{der}(14)$
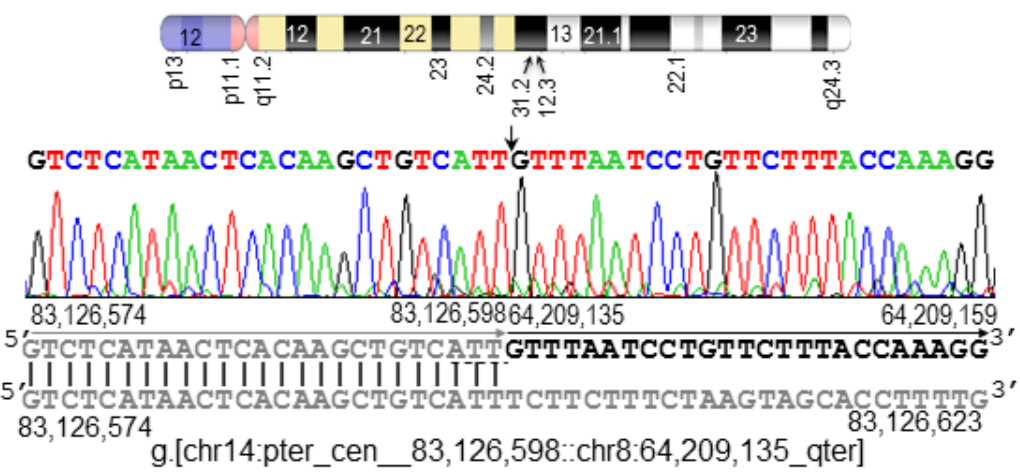

Supplementary Fig. 7 Nucleotide sequence of $\operatorname{der}(8)$ and der(14) breakpoints aligned against the GRCh38 reference human genome

a Chromosome 8q12.3 breakpoint. The chromosome 8 sequence is in black, whereas the chromosome 14 sequence is in gray. Vertical lines indicate identical nucleotides between derivative and reference chromosomes. The duplicated trinucleotide ATT sequence is boxed and in lowercase. Below, arrow within IVS 1 of LINC01414 indicates the position of the translocation breakpoint.

b Chromosome 14q31.2 breakpoint. The chromosome 8 sequence is in black, whereas the chromosome 14 sequence is in gray. Vertical lines indicate identical nucleotides between derivative and reference chromosomes. A dashed line underlines the duplicated trinucleotide.

The translocation is revised and described as seq[GRCh38] $46, X X, t(8 ; 14)(8 p t e r \rightarrow 8 q 12.3:: 14 q 31.2 \rightarrow 14 q$ ter; $14 p t e r \rightarrow 14 q 31.2:: 8 q 12.3 \rightarrow 8 q t e r) d n$. According to next-gen cytogenetics nomenclature (Ordulu et al. 2014), the translocation can be defined as $46, \mathrm{XX}, \mathrm{t}(8 ; 14)(\mathrm{q} 11.23 ; \mathrm{q} 24.3) \mathrm{dn}$.seq[GRCh38] $\mathrm{t}(8 ; 14)(8$ pter->q12.3(64,209,134):: $\quad$ ATT::14q31.2(83,126,599)->14qter;14pter$>14 q 31.2(83,126,598):: 8 q 12.3(64,209,135)->8 q t e r)$. 


\section{DGRC0013}

a

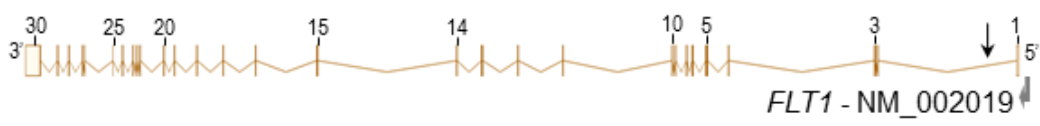

Proximal

5'AGCTMGTACCATAAGCCACAAGTATGTAAGCCACAGCTGTTCAGGAAAAG 3 '

5.AGCTAGTACCATAAGCCACAAGTATCTATAAATATAAATACTAGAGCATA 5"

$\overrightarrow{28,489,768 \quad 28,489,792} \quad \overrightarrow{74,831,804} 74,831,780$

AGCTAGTACCATAAGCCACAAGTATCTATAAATATAAATACTAGAGCATA

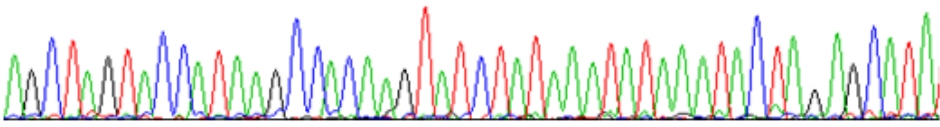

$\operatorname{inv}(13)$

b

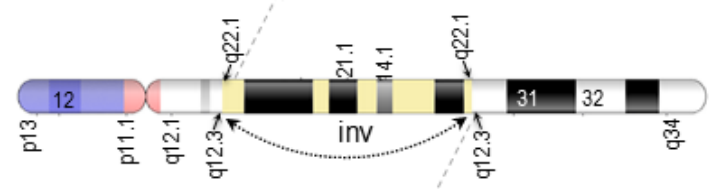

Distal

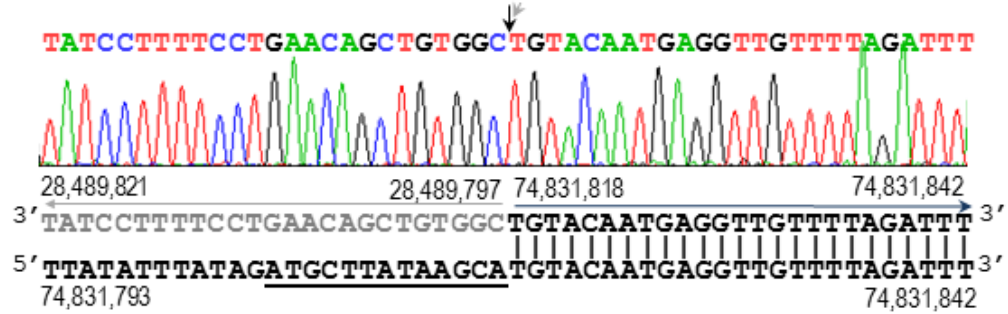

chr13:g.[pter-cen_28,489,793_28,489,795del;28,489,796_74,831,804inv;74,831,805_74,831,817del_qter]

Supplementary Fig. 8 Nucleotide sequence of the paracentric chromosome 13 inversion breakpoints aligned against the GRCh38 reference human genome a The proximal inversion breakpoint at $13 q 12.3$.

b The distal inversion breakpoint at 13q22.1.

Above, arrow within FLT1 IVS 1 indicates the position of the proximal inversion breakpoint.

Reference chromosome 13 sequences are in black, whereas the inverted chromosome 13 sequence is in gray. Vertical lines indicate identical nucleotides between the derivative and reference chromosome. The deleted GTA trinucleotide and the tridecanucleotide ATGCTTATAAGCA sequence from proximal and distal breakpoints, respectively, are underlined.

The inversion is revised and described as seq[GRCh38] 46,XY,inv(13)(pter $\rightarrow q 12.3:: q 22.1 \rightarrow q 12.3:: q 22.1 \rightarrow$ qter $) d n$.

According to next-gen cytogenetics nomenclature (Ordulu et al. 2014), the inversion is defined as 46,XY,inv(13)(q12.3q23)dn.seq[GRCh38] inv(13)(pter$>$ q12.3(28,489,792)::q22.1(-)(74,831,804)-

$>$ q12.3(28,489,795)::q22.1(+)(74,831,818)->qter $)$. 


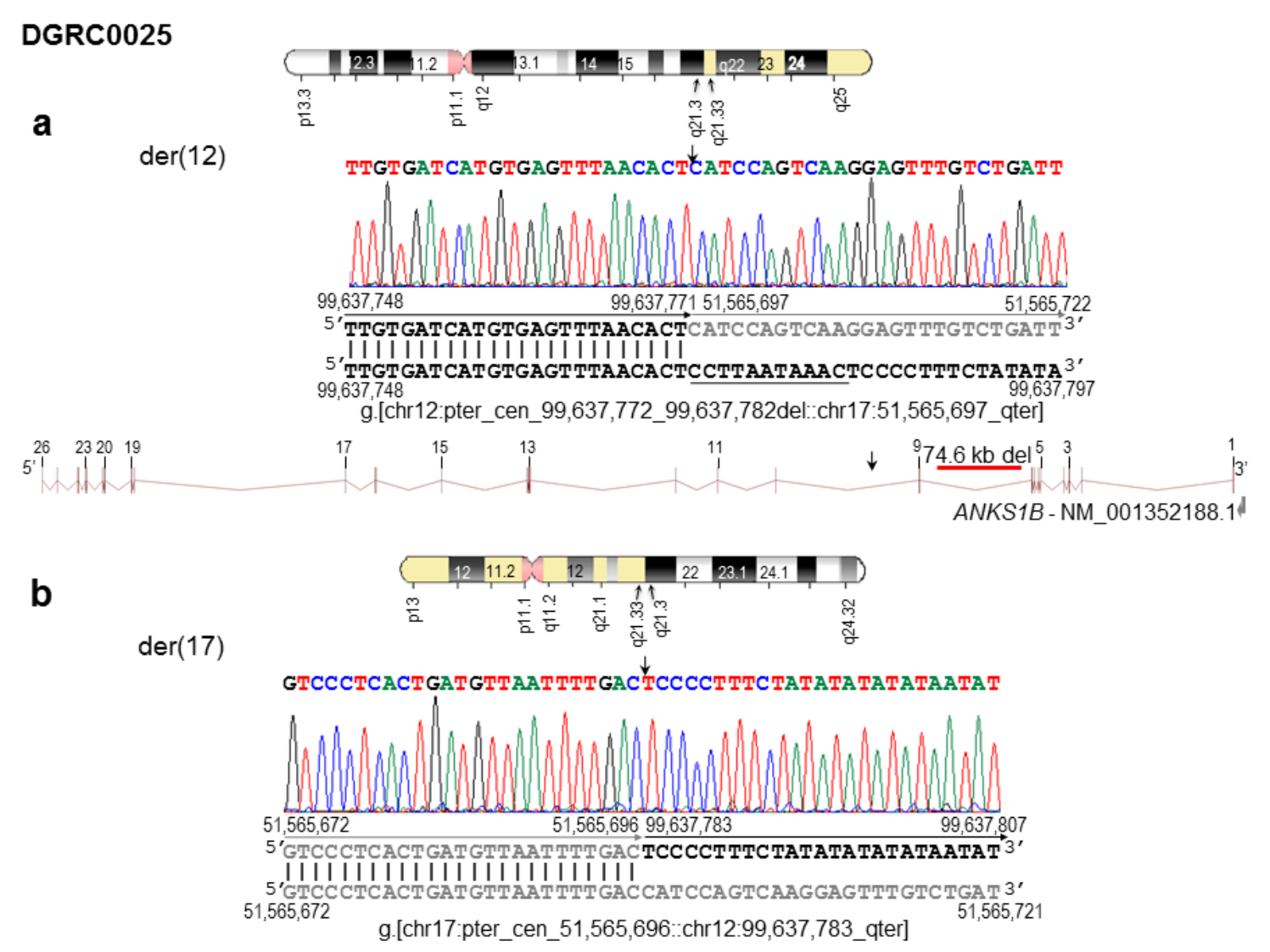

Supplementary Fig. 9 Nucleotide sequence of $\operatorname{der}(12)$ and $\operatorname{der}(17)$ breakpoints aligned against the GRCh38 reference human genome

a Chromosome 12q23.1 breakpoint. The chromosome 12 sequence is in black, whereas the chromosome 17 sequence is in gray. Vertical lines indicate identical nucleotides between derivative and reference chromosomes. The deleted hendecanucleotide CCTTAATAAAC sequence from the der(12) sequence is underlined.

Below, an arrow within IVS 9 and a red bar within IVS 8 of ANKRD11 indicate the position of the translocation breakpoint and of a $74.6 \mathrm{~Kb}$ deletion, respectively.

b Chromosome 17q21.33 breakpoint. The chromosome 12 sequence is in black, whereas the chromosome 17 sequence is in gray. Vertical lines indicate identical nucleotides between derivative and reference chromosomes.

The translocation is revised and described as seq[GRCh38] $46, X Y, t(12 ; 17)(12 p t e r \rightarrow 12 q 21.3:: 17 q 21.33 \rightarrow 17 q t e r ; 17 p t e r \rightarrow 17 q 21.33:: 12 q 21.3 \rightarrow 12$ qter)dn.

According to next-gen cytogenetics nomenclature (Ordulu et al. 2014), the translocation can be defined as 46,XY,t(12;17)(q23;q22)dn.seq[GRCh38] $\mathrm{t}(12 ; 17)(12 \mathrm{pter}->\mathrm{q} 23.1(99,637,771):: \mathrm{q} 23.1(99,637,782):: 17 \mathrm{q} 21.33(51,565,697)-$ $>17 q$ ter; 17 pter->q21.33(51,565,696)::12q23.1(99,637,783-99,680,962::99,755,562)$>12 q$ ter) 


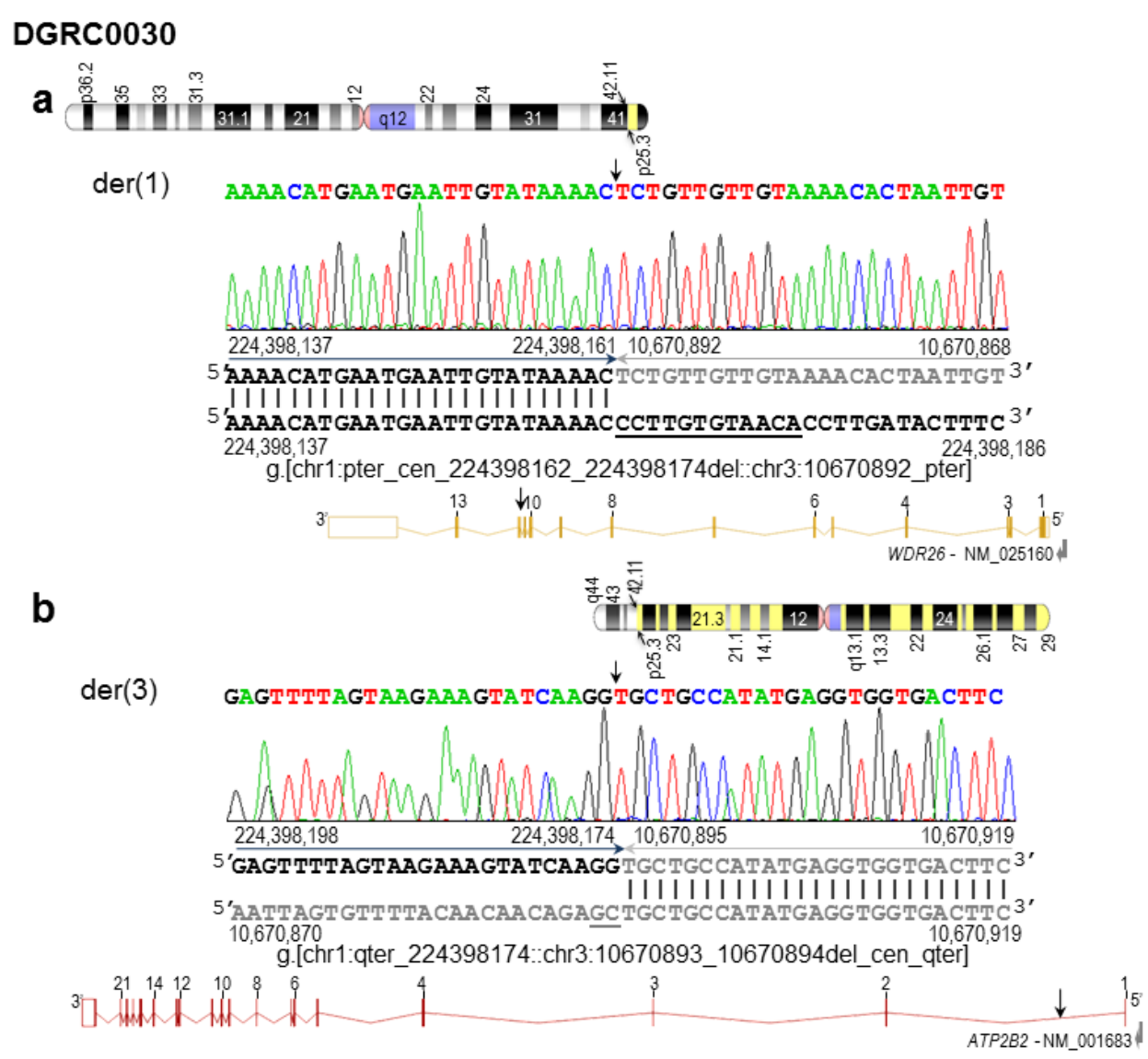

Supplementary Fig.10 Nucleotide sequence of der(1) and der(3) breakpoints aligned against the GRCh38 reference human genome

a Chromosome 1q42.11 breakpoint. The chromosome 1 sequence is in black, whereas the chromosome 3 sequence is in gray. Vertical lines indicate identical nucleotides between derivative and reference chromosomes. The deleted dodecanucleotide CCTTGTGTAACA sequence from der(1) sequence is underlined. Below, arrow within exon 12 of WDR12 indicates the translocation breakpoint.

b Chromosome 3p25.3 breakpoint. The chromosome 1 sequence is in black, whereas the chromosome 3 sequence is in gray. Vertical lines indicate identical nucleotides between derivative and reference chromosomes. The deleted GC dinucleotide from der(3) sequence is underlined. Below, arrow within IVS 1 ATP2B2 indicates the translocation breakpoint.

The translocation is revised and described as seq[GRCh38] $46, X Y, t(1 ; 3)(1$ pter $\rightarrow 1 \mathrm{q} 42.11:: 3 p 25.3 \rightarrow 3 p t e r ; 1 q t e r \rightarrow 1 q 42.11:: 3 p 25.3 \rightarrow 3 q t e r) d n$.

According to next-gen cytogenetics nomenclature (Ordulu et al. 2014), the translocation can be defined as $46, \mathrm{XY}, \mathrm{t}(1 ; 3)(\mathrm{q} 42 ; \mathrm{p} 25) \mathrm{dn}$.seq[GRCh38] $\mathrm{t}(1 ; 3)(1$ pter$>$ q42.11(224,398,161)::3p25.3(-)(10,670,892)->3pter;1qter->q42.11()(224,398, 174)::3p25.3(+)(10,670,895)->3qter) 


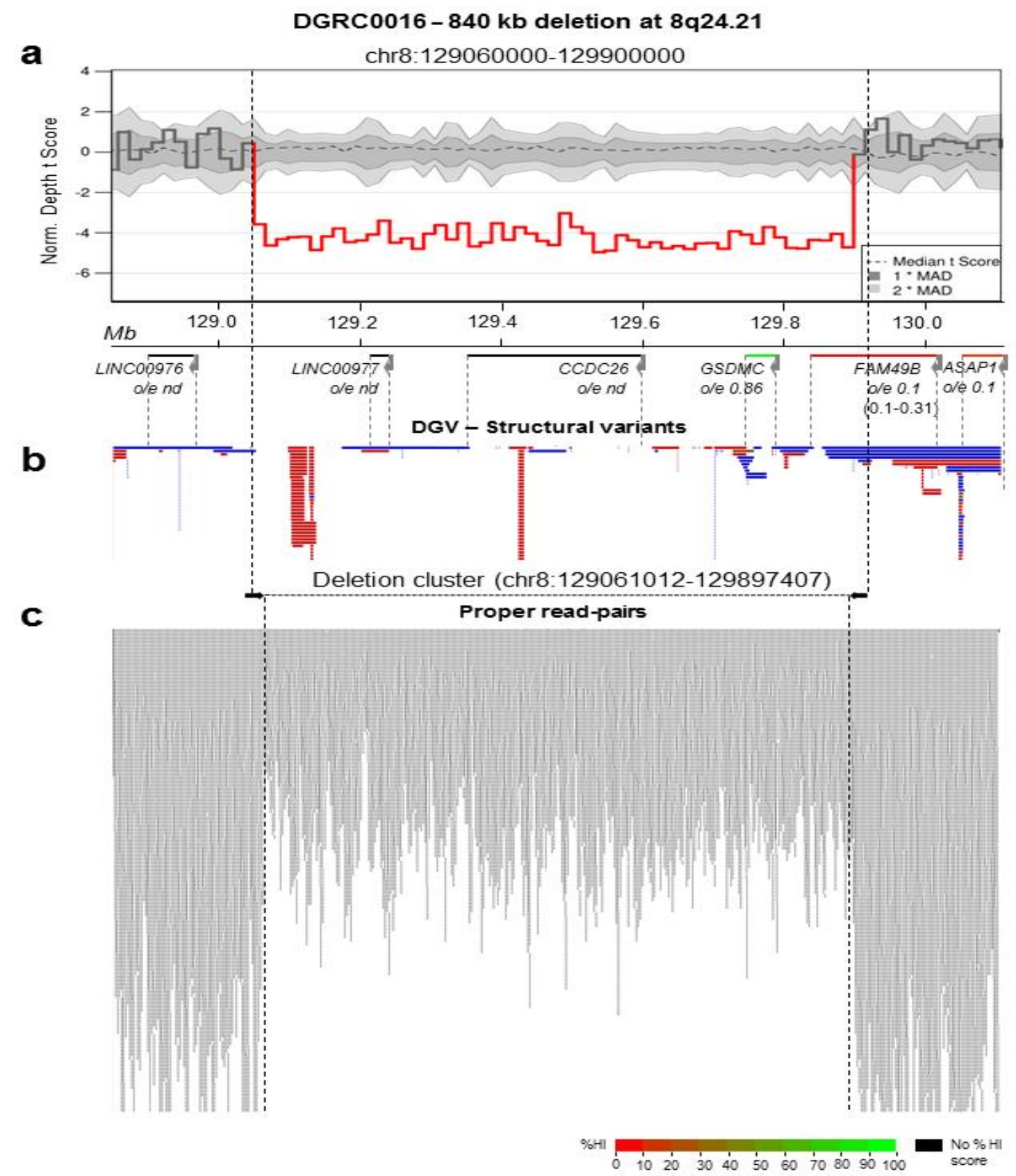

Supplementary Fig. 11 Illustration of DGRC0016-specific genomic imbalance identified at 8q24.21 by sequence coverage and read-pair cluster analysis

a Genomic imbalance plot generated by CNView (Collins et al. 2016) showing an $840 \mathrm{~kb}$ del. A group of 32 cases, analyzed using the same type of liGs library, was used as control. The horizontal black dashed line with darker and lighter gray shading indicate median coverage and deviation, respectively. Regions with a statistically significant decrease in sequence coverage ( $\alpha=0.05$, Bonferroni correction) indicating dels are depicted in red. Gene map across this region is shown below. Forward and reverse folded arrows indicate the position of the genes in sense and antisense orientation, respectively. Genes are color coded according to their haploinsufficiency index $(\mathrm{HI})$ and LoF intolerance, expressed as observed / expected (oe) number of LoF variants, are stated below the genes. Confidence interval of $F A M 49 B$ oe is indicated in parentheses.

b CNVs from the affected genomic region reported in the DGV database (MacDonald et al. 2014). Blue and red bars represent gains and losses, respectively. Vertical dashed black lines delimitate the overlap between the del with gene and CNVs.

c Read-pair cluster delimiting the del and proper read-pairs aligned within the involved genomic region. Black arrows depict inward-facing read-pair cluster delimiting the del. Below, small gray vertical bars denote proper read-pairs mapped within this region, illustrating the decrease in coverage within the deleted region. 
a

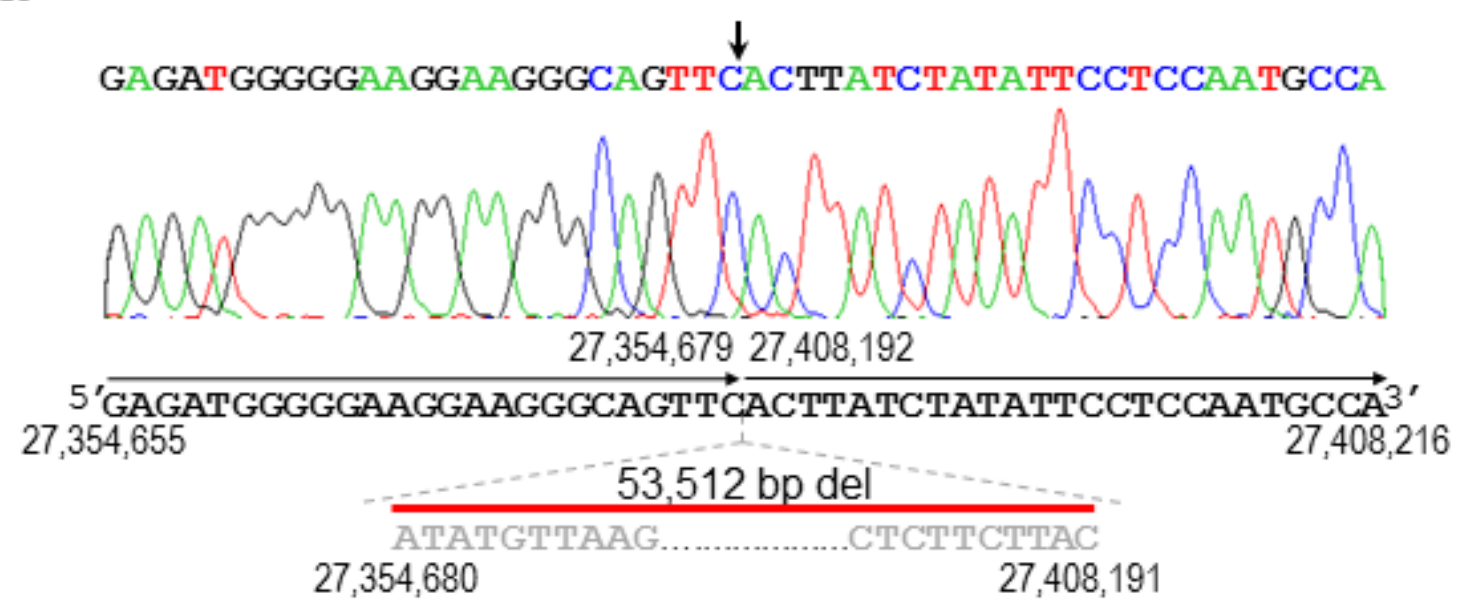

b

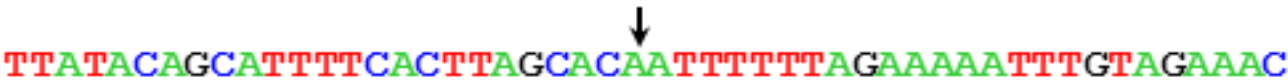

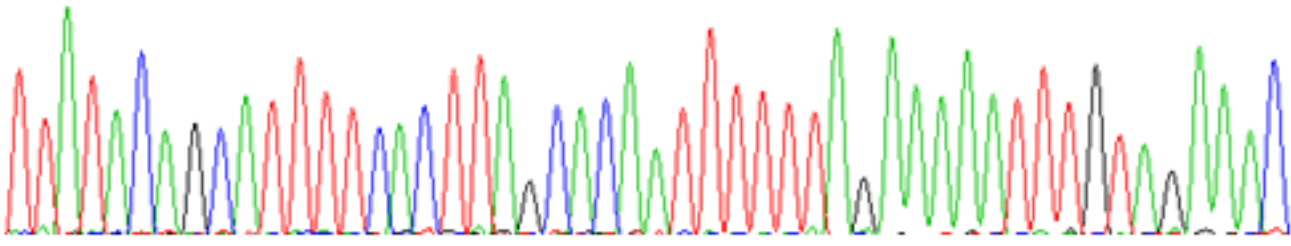
$129,061,232 \quad 129,897,282$

5 'TTATACAGCATTTTCACTTAGCACA $2 \overrightarrow{2 T T T T A G A A A A A T T T G T A G A A A C}{ }^{3}$ $129,061,208$

836,049 bp del

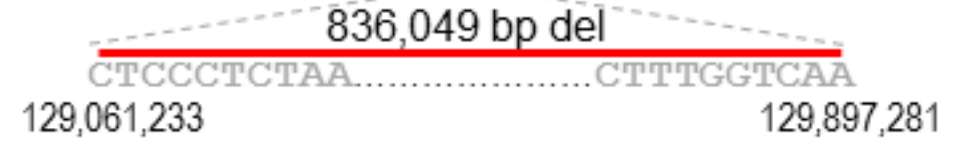

Supplementary Fig. 12 Nucleotide sequence of genomic imbalance breakpoints at 3p24.1 and 8q24.21 identified in DGRC0016

a The 53,512 bp del breakpoint at 3p24.1.

b The 836,049 bp del breakpoint at 8q24.21.

Vertical arrows indicate positions of del breakpoints, whereas horizontal arrows denote orientation of the genomic sequence. Deleted sequences at breakpoint junctions are in gray and dels are depicted by red bars. The inserted AT dinucleotide at the breakpoint is boxed and in lowercase. 

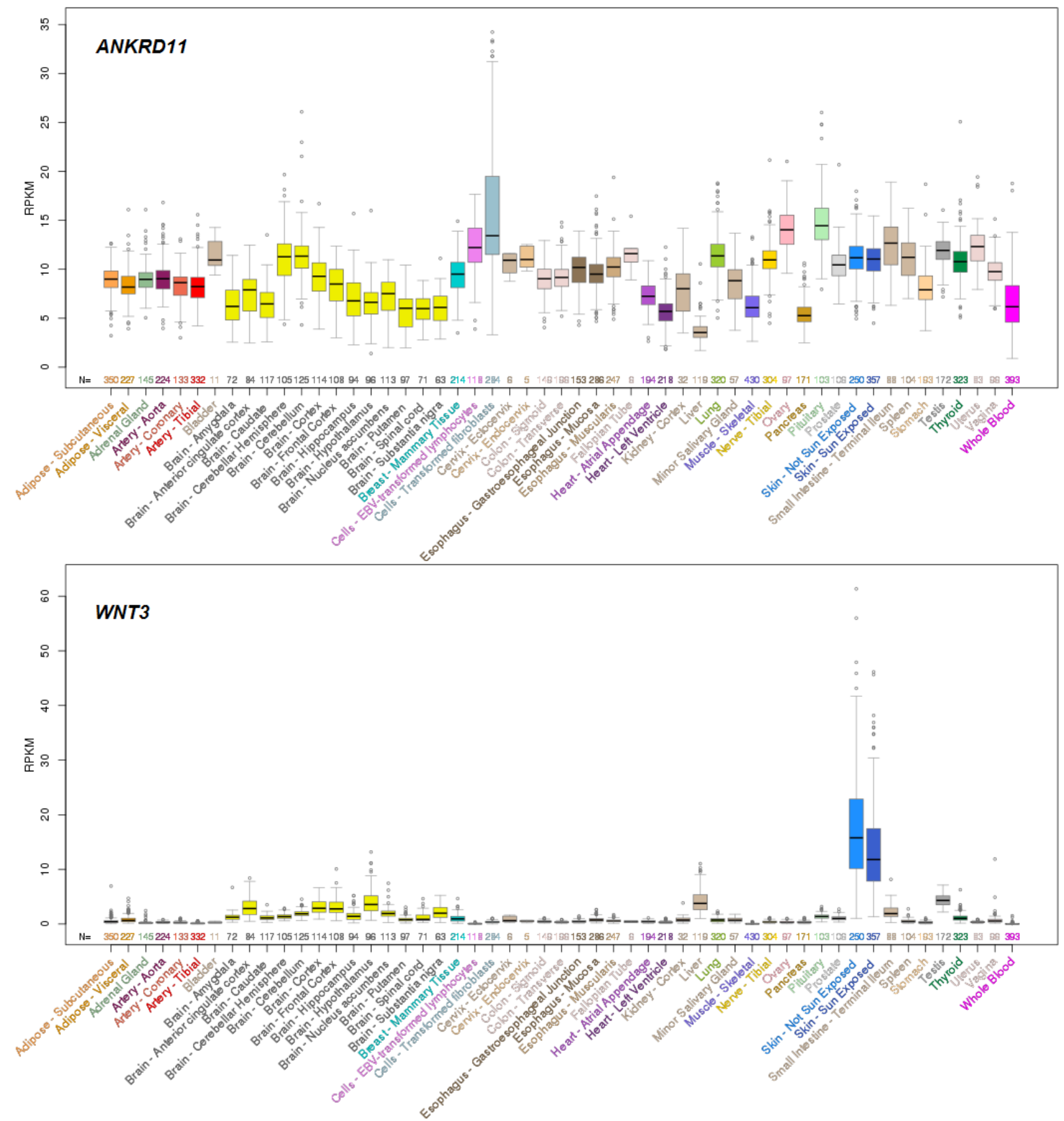

Supplementary Fig. 13 Tissue-specific expression profiles of ANKRD11 and WNT3 disrupted by the 16q24.3 and 17q21.31 breakpoints, respectively (from Genotype-Tissue Expression, GTEx) 


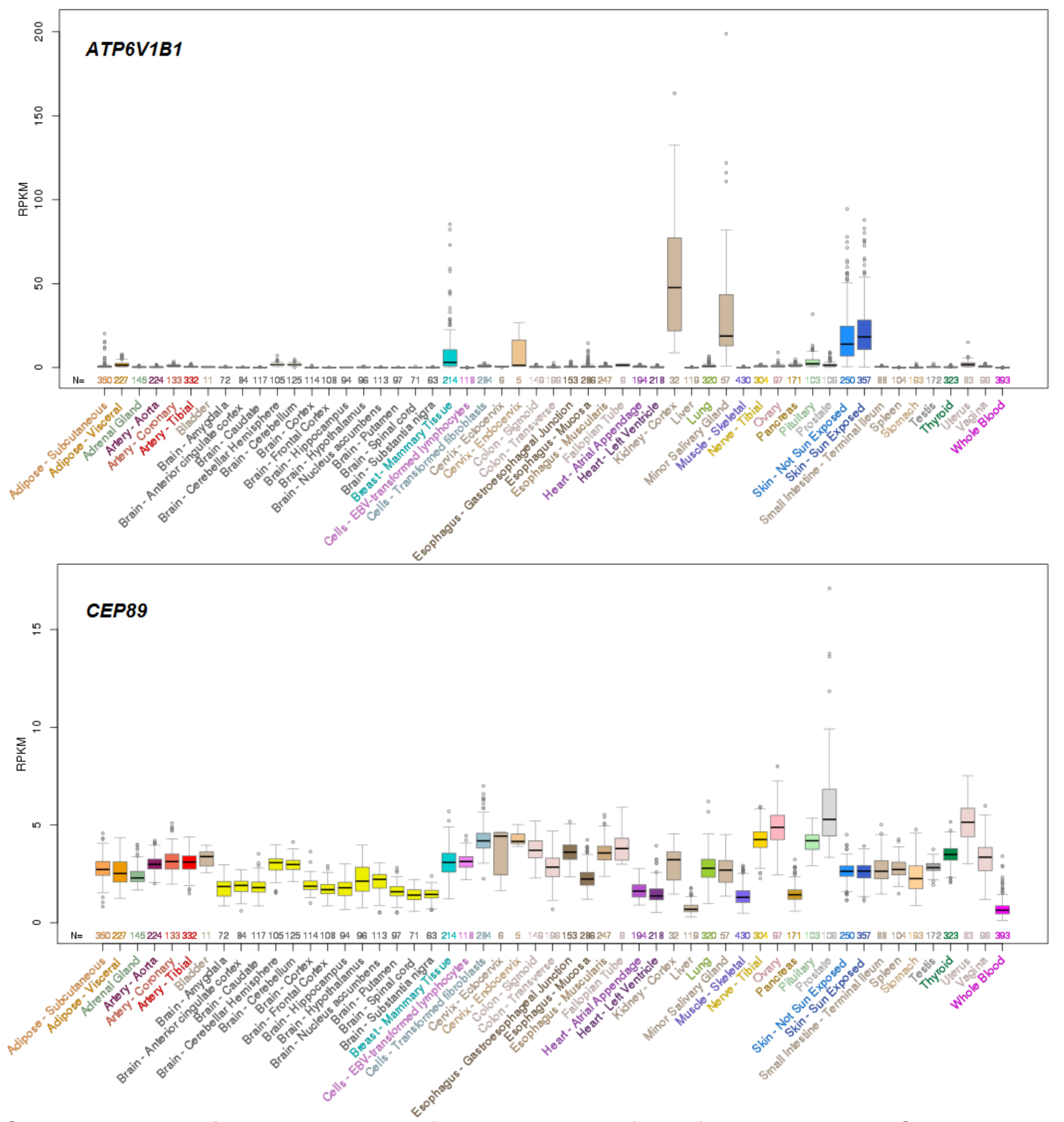

Supplementary Fig. 14 Tissue-specific expression profiles of ATP6V1B1 and CEP89 (from Genotype-Tissue Expression, GTEx)

The 2p13.3 breakpoint is within IVS1 of ATP6V1B1 whereas that of $19 q 13.11$ is within the 3' UTR of CEP89. 


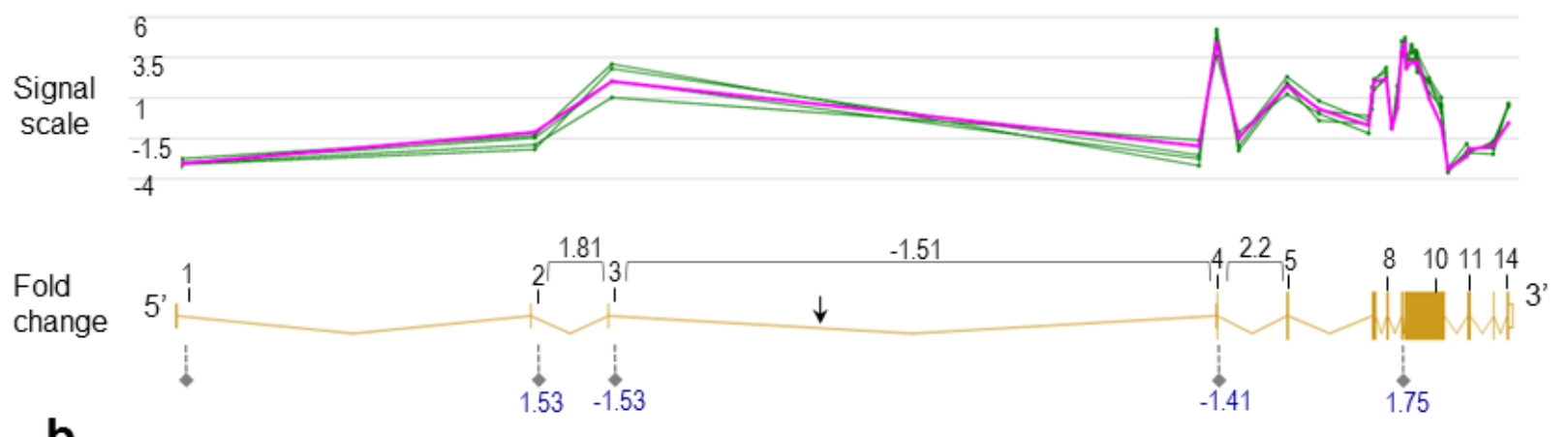

b

Sample-level signals WNT3- NM_03075 -

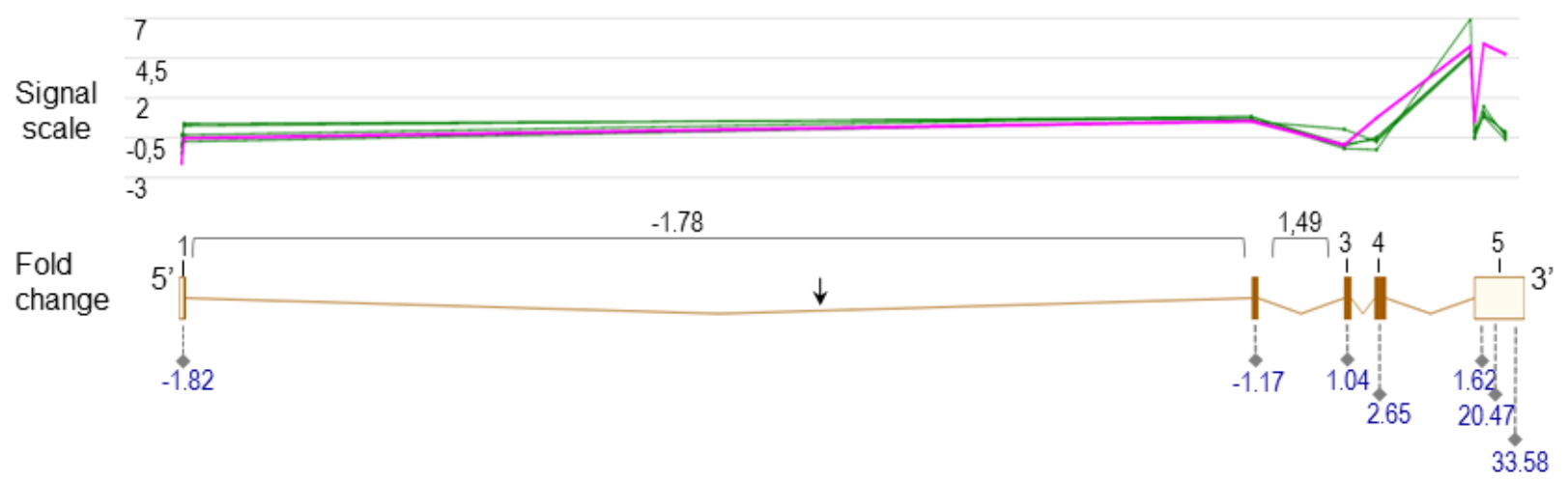

Supplementary Fig. 15 Differential expression at exon and exon junction levels of the $t(16 ; 17)$ disrupted genes

a Gene structure-based display of sample-level signal intensities of the disrupted ANKRD11. Below, schematic gene map with exons and exon junction fold changes.

b Gene structure-based display of sample-level signal intensities of the disrupted WNT3. Below, schematic gene map with exons and exon junction fold changes.

The detection threshold of HTA 2.0 microarray is a two-fold change. Control LCL samples are in green and the proband's LCL sample is in purple. Exons are numbered. Under the gene maps, dashed lines with filled diamonds indicate exons and the corresponding probe set fold change between the proband and the control samples. Above the gene map, horizontal square brackets show exon junction fold changes between the designated exons. Arrows indicate the breakpoints. 


\section{Supplementary Tables}

Supplementary Table 1 Primers used for validation of structural chromosomal abnormalities and CNVs

\begin{tabular}{|c|c|c|c|c|c|}
\hline Fragments & Designation & Primer sequence ( $\left.5^{\prime}-3^{\prime}\right)$ & Primer localization a & $\begin{array}{l}\text { Annealing } \\
T\left(C^{\circ}\right)\end{array}$ & $\begin{array}{l}\text { Amplicon } \\
\text { length (bp) }\end{array}$ \\
\hline \multicolumn{6}{|c|}{ DGRC0016 } \\
\hline $\begin{array}{l}\text { Control fragment } \\
\text { chr16 }\end{array}$ & $\begin{array}{l}\text { ANKRD11_IVS3-1F } \\
\text { ANKRD11_IVS3-2R }\end{array}$ & $\begin{array}{l}\text { CCACCTCCCATCCACACCT } \\
\text { GTACCCAGAGAGGGTCAGC }\end{array}$ & $\begin{array}{l}\text { Chr16:89,401,547-89,401,566 } \\
\text { Chr16:89,401,821-89,401,840 }\end{array}$ & 61 & 295 \\
\hline $\begin{array}{l}\text { Control fragment } \\
\text { chr17 }\end{array}$ & $\begin{array}{l}\text { WNT3_IVS1-3F } \\
\text { WNT3_IVS1-2R }\end{array}$ & $\begin{array}{l}\text { TAGCTGGGCTTTGGAATATCAT } \\
\text { GCAACACAAACCTCTACCCCTA }\end{array}$ & $\begin{array}{l}\text { Chr17:46,783,899-46,783,920 } \\
\text { Chr17:46,784,399-46,784,418 }\end{array}$ & 61 & 520 \\
\hline $\begin{array}{l}\text { Junction fragment } \\
\text { der(16) }\end{array}$ & $\begin{array}{l}\text { ANKRD11_IVS3-1F } \\
\text { WNT3_IVS1-2R }\end{array}$ & 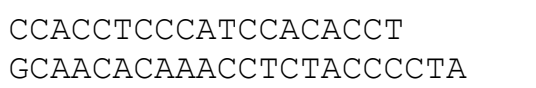 & $\begin{array}{l}\text { Chr16:89,401,547-89,401,566 } \\
\text { Chr17:46,784,399-46,784,418 }\end{array}$ & 61 & 559 \\
\hline $\begin{array}{l}\text { Junction fragment } \\
\text { der(17) } \\
\text { seq[GRCh38] chr3: }\end{array}$ & $\begin{array}{l}\text { WNT3_IVS1-1F } \\
\text { ANKRD11_IVS3-2R } \\
\text { g.27354680_27408191del }\end{array}$ & $\begin{array}{l}\text { CAGCCTACCCCTTACTTTTCAC } \\
\text { GTACCCAGAGAGGGGTCAGC }\end{array}$ & $\begin{array}{l}\text { Chr17:46,781,765-46,781,785 } \\
\text { Chr16:89,401,821-89,401,840 }\end{array}$ & 61 & 357 \\
\hline $\begin{array}{l}\text { Control fragment } \\
\text { proximal }\end{array}$ & $\begin{array}{l}\text { SLC4A7_IVS13-1F } \\
\text { SLC4A7_IVS13-2R }\end{array}$ & $\begin{array}{l}\text { ACACACTCTGGGACCGTATCT } \\
\text { CTGTGGCTTTTTGAAGTGGCAT }\end{array}$ & $\begin{array}{l}\text { Chr3:27,407,917-27,407,937 } \\
\text { Chr3:27,408,859-27,408,880 }\end{array}$ & 63 & 964 \\
\hline $\begin{array}{l}\text { Control fragment } \\
\text { distal }\end{array}$ & $\begin{array}{l}\text { NEK10_IVS2-1F } \\
\text { NEK10_IVS2-2R }\end{array}$ & $\begin{array}{l}\text { CCAAGTCTCTCAGCACAGGAG } \\
\text { GCAGAAGAAGCCTGTGAGCTT }\end{array}$ & $\begin{array}{l}\text { Chr3:27,353,627-27,353,647 } \\
\text { Chr3:27,354,222-27,354,242 }\end{array}$ & 63 & 606 \\
\hline $\begin{array}{l}\text { Deletion junction } \\
\text { fragment } \\
\text { seq[GRCh38] chr8: }\end{array}$ & $\begin{array}{l}\text { NEK10_IVS2-1F } \\
\text { SLC4A7 IVS13-2R } \\
\text {.129061233 129897281del }\end{array}$ & $\begin{array}{l}\text { CCAAGTCTCTCAGCACAGGAG } \\
\text { CTGTGGCTTTTTGAAGTGGCAT }\end{array}$ & $\begin{array}{l}\text { Chr3:27,353,627-27,353,647 } \\
\text { Chr3:27,408,859-27,408,880 }\end{array}$ & 63 & 1,741 \\
\hline $\begin{array}{l}\text { Control fragment } \\
\text { proximal }\end{array}$ & $\begin{array}{l}\text { AC104256-3F } \\
\text { AC104256-4R }\end{array}$ & $\begin{array}{l}\text { TAGGGAAACCAAGTTCAGGCTC } \\
\text { GGTGAAACACAGATGATGCTCC }\end{array}$ & $\begin{array}{l}\text { Chr8:129,060,775-129,060,796 } \\
\text { Chr8:129,061,434-129,061,455 }\end{array}$ & 60 & 681 \\
\hline $\begin{array}{l}\text { Control fragment } \\
\text { distal }\end{array}$ & $\begin{array}{l}\text { FAM49B-1F } \\
\text { FAM49B-3R }\end{array}$ & $\begin{array}{l}\text { ACCTCCCCAGAGTGAGAGTC } \\
\text { TATAGGCATTAGCCGCCCAC }\end{array}$ & $\begin{array}{l}\text { Chr8:129,896,671-129,896,689 } \\
\text { Chr8:129,897,660-129,897,679 }\end{array}$ & 60 & 1,010 \\
\hline $\begin{array}{l}\text { Deletion junction } \\
\text { fragment }\end{array}$ & $\begin{array}{l}\text { AC104256-3F } \\
\text { FAM49B-3R }\end{array}$ & $\begin{array}{l}\text { TAGGGAAACCAAGTTCAGGCTC } \\
\text { TATAGGCATTAGCCGCCCAC }\end{array}$ & $\begin{array}{l}\text { Chr8:129,060,775-129,060,796 } \\
\text { Chr8:129,897,660-129,897,679 }\end{array}$ & 60 & 858 \\
\hline \multicolumn{4}{|c|}{ DGRC0019 } & & \\
\hline \multirow{2}{*}{$\begin{array}{c}\text { Control fragment } \\
\text { chr2 } \\
\text { Control fragment } \\
\text { chr19 } \\
\text { Junction fragment } \\
\text { der(2) }\end{array}$} & $\begin{array}{l}\text { ATP6V1B1-1F } \\
\text { ATP6V1B1-2R } \\
\text { A008805-1F } \\
\text { A008805-2R } \\
\text { ATP6V1B1-2R }\end{array}$ & $\begin{array}{l}\text { AGGTGTGAGCCACTGTAGCTG } \\
\text { GACTCACACTCCTCGCTCTCAG } \\
\text { AGCAGCACCTTGTTTTTTTTG } \\
\text { TCTGTTTTAGCCAGGGCATG } \\
\text { GACTCACACTCCTCGCTCTCAG }\end{array}$ & $\begin{array}{l}\text { Chr2:70,941,481-70,941,502 } \\
\text { Chr2:70,941,807-70,941,828 } \\
\text { Chr19:32,878,365-32,878,385 } \\
\text { Chr19:32,878,632-32,878,651 } \\
\text { Chr2·70 } 941807-70941828\end{array}$ & 61 & 635 \\
\hline & $\begin{array}{l}A 008805-2 R \\
A 008805-1 F\end{array}$ & $\begin{array}{l}\text { TCTGTTTTAGCCAGGGCATG } \\
\text { AGCAGCACCTTGTTTTTTTTG }\end{array}$ & $\begin{array}{l}\text { Chr19:32,878,632-32,878,651 } \\
\text { Chr19:32,878,365-32,878,385 }\end{array}$ & 61 & 459 \\
\hline
\end{tabular}




\section{DGRC0006}

\begin{tabular}{cc}
$\mathrm{t}(8 ; 14)(\mathrm{q} 12.3 ; \mathrm{q} 31.2)$ & \\
\hline Control fragment & $\mathrm{AC} 023533-1 \mathrm{~F}$ \\
chr8 & $\mathrm{AC023533-2R}$ \\
Control fragment & $\mathrm{AL} 162872-1 \mathrm{~F}$ \\
chr14 & $\mathrm{AL} 162872-3 \mathrm{R}$ \\
Junction fragment & $\mathrm{AC} 023533-1 \mathrm{~F}$ \\
der(8) & $\mathrm{AL} 162872-3 \mathrm{R}$ \\
Junction fragment & $\mathrm{AL} 162872-1 \mathrm{~F}$ \\
der(14) & $\mathrm{AC023533-2R}$ \\
\hline
\end{tabular}

TGGAGAGCAAGGCAAACAGT CCCTTGAAACTCCCCAGACT AGATGTTTGGCGTAGGCTTG CACAGGGATTTGGTTTATCATTCCT TGGAGAGCAAGGCAAACAGT CACAGGGATTTGGTTTATCATTCCT AGATGTTTGGCGTAGGCTTG

\section{DGRC0013}

$\operatorname{inv}(13)(q 12.3 q 22.1)$

Control fragment FLT1-IVS1-1F

proximal chr13 FLT1-IVS1-3R

Control fragment AL590063-1F distal chr13

AL590063-2R

Junction fragment FLT1-IVS1-1F

proximal chr13 AL590063-1F

Junction fragment AL590063-2R distal chr13

AGAGATGGTGATTACTGGGCTG ACTGGTGTATTTCCTCTTCCC TGAATCCACAGCATCTCCAAG TGTTTCAGGGTTGCTTTCTTG AGAGATGGTGATTACTGGGCTG TGAATCCACAGCATCTCCAAG TGTTTCAGGGTTGCTTTCTTG ACTGGTGTATTTCCTCTTCCC

\section{DGRC0025}

$\mathrm{t}(12 ; 17)(\mathrm{q} 23.1 \mathrm{q} 21.33)$

Control fragment AC141554-3F

$$
\text { chr12 }
$$

AC141554-2R

Control fragment AC005883-1F chr17

AC005883-2R

Junction fragment AC141554-3F $\operatorname{der}(12)$ AC005883-2R Junction fragment AC005883-1F $\operatorname{der}(17)$

AC141554-2R

$\mathrm{t}(1 ; 3)(\mathrm{q} 42.11 \mathrm{p} 25.3)$

Control fragment WDR26-1F chr1

WDR26-2R

Control fragment ATPB2B2-1F chr3 ATPB2B2-2R

Junction fragment WDR26-1F $\operatorname{der}(1)$
TGGGCAGGCGCAATCTAAT GGGTAACCATCCCCGTGATT TGTCACGTCTCTTTAGTCTCCTT TAGCCCAGTTTCCGCTCATT TGGGCAGGCGCAATCTAAT TAGCCCAGTTTCCGCTCATT TGTCACGTCTCTTTAGTCTCCTT GGGTAACCATCCCCGTGATT

\section{DGRC0030}

AGCACTGCTAATCCGTGACA ATCTGTATTCTCCTTTGCCTGC TCTAAAGGATGTTCGTGTGGAAG ACTCCACCCCTTTTACCACC AGCACTGCTAATCCGTGACA ACTCCACCCCTTTTACCACC
Chr8:64,208,781-64,208,800

Chr8:64,209,331-64,209,350

Chr14:83,126,183-83,126,202

Chr14:83,126,626-83,126,650

Chr8:64,208,781-64,208,800

Chr14:83,126,626-83,126,650

Chr14:83,126,183-83,126,202

Chr8:64.209.331-64.209.350

Chr13:28,489,561-28,489,582 Chr13:28,490,028-28,490,048 Chr13:74,831,722-74,831,742

Chr13:74,832,041-74,832,061

Chr13:28,489,561-28,489,582

Chr13:74, 831,722-74, 831,742

Chr13:74,832,041-74,832,061

Chr13:28,490,028-28,490,048

\author{
60
}

1,154

60

487

60

315

60

496

\section{Chr12:99,637,540-99,637,561}

Chr12:99,637,964-99,637,983

Chr17:51,565,437-51,565,459

Chr17:51,566,189-51,566,208

Chr12:99,637,540-99,637,561

Chr17:51,566,189-51,566,208

Chr17:51,565,437-51,565,459

Chr12:99.637.964-99.637.983

64

64

63

63

443

771

44

461

Chr1:224,397,833-224,397,852 Chr1:224,398,229-224,398,250 Chr3:10,670,741-10,670,763

Chr3:10,671,232-10,671,251

Chr1:224,397,833-224,397,852

Chr3:10,671,232-10,671,251
62

418

62

62 
Junction fragment ATPB2B2-1F

WDR26-2R

TCTAAAGGATGTTCGTGTGGAAG

Chr3:10,670,741-10,670,763

Chr1:224.398.229-224.398.250

a Reference genome assembly GRCh38/hg38. 
Supplementary Table 2 Comparison of the proband's clinical features with those of patients with pathogenic SNVs, deletions and intragenic duplications of ANKRD11 and 16q24.3 microdeletions encompassing ANKRD11

\begin{tabular}{|c|c|c|c|c|c|}
\hline Clinical features & HPO & $\begin{array}{c}\text { Proband's } \\
\text { phenotype } \\
\text { t(16;17) }\end{array}$ & $\begin{array}{l}\text { Pathogenic variants } \\
\text { /deletion/intragenic } \\
\text { dup }\end{array}$ & $\begin{array}{c}\text { 16q24.3 microdeletions } \\
\text { encompassing ANKRD11 } \\
\mathrm{N}=36\end{array}$ & $\begin{array}{l}\text { Prenatal case of } \\
\text { KBG by deletion } \\
16 q 24.2 q 24.3\end{array}$ \\
\hline $\begin{array}{l}\text { Neurologic } \\
\text { Global developmental delay } \\
\text { Seizures } \\
\text { Abnormality of brain morphology }\end{array}$ & $\begin{array}{l}\text { HP:0001263 } \\
\text { HP:0001250 } \\
\text { HP:0012443 }\end{array}$ & $\begin{array}{l}\text { Yes } \\
\text { No } \\
\text { Unknown } \\
\end{array}$ & $\begin{array}{l}16 / 40(40 \%) \\
8 / 40(20 \%) \\
1 / 40(2.5 \%) \\
\end{array}$ & $\begin{array}{l}30 / 36(\sim 83 \%) \\
10 / 36(\sim 28 \%) \\
10 / 36(\sim 28 \%) \\
\end{array}$ & $\begin{array}{l}\text { NA } \\
\text { NA } \\
\text { Not reported }\end{array}$ \\
\hline $\begin{array}{l}\text { Craniofacial } \\
\text { Microcephaly } \\
\text { Low posterior hairline } \\
\text { Round face early in life } \\
\text { Long philtrum } \\
\text { Anteverted nares } \\
\text { Underdeveloped nasal alae } \\
\text { Protruding ear } \\
\text { Hypertelorism } \\
\text { Telecanthus } \\
\text { Long palpebral fissures } \\
\text { Thick eyebrows } \\
\end{array}$ & $\begin{array}{l}\text { HP:0000252 } \\
\text { HP:0002162 } \\
\text { HP:0000311 } \\
\text { HP:0000343 } \\
\text { HP:0000463 } \\
\text { HP:0000430 } \\
\text { HP:0000411 } \\
\text { HP:0000316 } \\
\text { HP:0000506 } \\
\text { HP:0000637 } \\
\text { HP:0000574 }\end{array}$ & $\begin{array}{l}\text { No } \\
\text { Yes } \\
\text { Yes } \\
\text { No } \\
\text { No } \\
\text { No } \\
\text { Yes } \\
\text { Yes } \\
\text { No } \\
\text { No } \\
\text { No }\end{array}$ & $\begin{array}{l}\text { Characteristic facial } \\
\text { anomalies } \\
40 / 40(100 \%)\end{array}$ & $\begin{array}{l}\text { Characteristic facial } \\
\text { anomalies } \\
16 / 36(\sim 44 \%)\end{array}$ & $\begin{array}{c}\text { Triangular face } \\
\text { Mildly low-seat ears }\end{array}$ \\
\hline $\begin{array}{l}\text { Growth } \\
\text { Postnatal growth retardation }\end{array}$ & HP:0008897 & Yes & $27 / 40(675 \%)$ & $12 / 36(\sim 33 \%)$ & Yes \\
\hline $\begin{array}{l}\text { Hand anomalies } \\
\text { Single transverse palmar crease } \\
\text { Finger clinodactyly } \\
\text { Finger syndactyly }\end{array}$ & $\begin{array}{l}\mathrm{HP}: 0000954 \\
\mathrm{HP}: 0040019 \\
\mathrm{HP}: 0006101\end{array}$ & $\begin{array}{l}\text { Yes } \\
\text { Yes } \\
\text { No }\end{array}$ & $\begin{array}{c}\text { Hand anomalies } \\
35 / 40(87.5 \%)\end{array}$ & $\begin{array}{c}\text { Hand anomalies } \\
\text { 10/36 ( 28\%) }\end{array}$ & Not reported \\
\hline $\begin{array}{l}\text { Skeletal } \\
\text { Delayed skeletal maturation } \\
\text { Thoracic kyphosis } \\
\text { Vertebral fusion } \\
\text { Vertebral arch anomaly } \\
\text { Accessory cervical ribs } \\
\end{array}$ & $\begin{array}{l}H P: 0002750 \\
H P: 0002942 \\
H P: 0002948 \\
H P: 0008438 \\
H P: 0000891\end{array}$ & $\begin{array}{l}\text { Unknown } \\
\text { No } \\
\text { Unknown } \\
\text { Unknown } \\
\text { Unknown }\end{array}$ & $\begin{array}{c}12 / 40(30 \%) \\
\text { Costovertebral anomalies } \\
17 / 40(42.5 \%)\end{array}$ & $\begin{array}{c}10 / 36(\sim 28 \%) \\
\text { Costovertebral anomalies } \\
0 / 36(0 \%)\end{array}$ & $\begin{array}{l}\text { Cervical ribs } \\
\text { Irregular C1-C4 } \\
\text { vertebral bodies }\end{array}$ \\
\hline $\begin{array}{ll}\text { Eyes } & \\
& \text { Astigmatism } \\
\end{array}$ & HP:0000483 & Unknown & $0 / 40(0 \%)$ & $10 / 36(\sim 28 \%)$ & Not reported \\
\hline $\begin{array}{l}\text { Cardiovascular } \\
\text { Congenital heart defect }\end{array}$ & HP:0001627 & Yes & $5 / 40(12.5 \%)$ & $12 / 36(\sim 33 \%)$ & Not reported \\
\hline $\begin{array}{l}\text { Hematopoietic system } \\
\text { Thrombocytopenia }\end{array}$ & HP:0001873 & Unknown & $0 / 40(0 \%)$ & $8 / 36(\sim 22 \%)$ & Not reported \\
\hline
\end{tabular}


Pathogenic variants of $A N K R D 11$ and 16q24.3 microdeletions are according to Novara et al. (2017). Prospective antenatal diagnosis of KBG syndrome by a $1.86 \mathrm{Mb}$ deletion, arr[GRCh37] 16q24.2q24.3(87614996_89479537)x1 dn, containing 26 genes including ANKRD11 reported in Hodgetts et al. (2017). Pregnancy was terminated at 27 weeks; additional features at postmortem examination included lobation of the left lung, lobulated spleen and portal tracts calcification of the liver. 
Supplementary Table 3 Comparison of the DGRC0025 - $t(12 ; 17)$ proband's clinical features with those of patients with $A N K S 1 B$ haploinsufficiency syndrome

\begin{tabular}{|c|c|c|c|}
\hline Clinical Features & $\begin{array}{c}\mathrm{HPO} \\
\text { nomenclature }\end{array}$ & $\begin{array}{c}\text { Proband's } \\
\text { phenotype } \\
t(12 ; 17)(q 23 ; q 22) \\
\end{array}$ & $\begin{array}{c}\text { ANKS1B } \\
\text { microdeletion } \\
\mathrm{n}=9(\%)\end{array}$ \\
\hline \multicolumn{4}{|l|}{ Craniofacial } \\
\hline Brachycephaly & HP:0000248 & No & $3 / 9(33 \%)$ \\
\hline Positional plagiocephaly & HP:0001357 & No & $1 / 9(11 \%)$ \\
\hline Round face & HP:0000311 & No & $4 / 9(44 \%)$ \\
\hline Prominent brow & HP:0000336 & No & $1 / 9(11 \%)$ \\
\hline Prominent metopic ridge & HP:0005487 & No & $1 / 9(11 \%)$ \\
\hline Congenital glabellar hemangioma & HP:0001076 & Yes & - \\
\hline Midface hypoplasia & HP:0011800 & No & $3 / 9(33 \%)$ \\
\hline Hypertelorism & HP:0000316 & No & $1 / 9(11 \%)$ \\
\hline Synophrys & HP:0000664 & No & $1 / 9(11 \%)$ \\
\hline Downslanted palpebral fissure & HP:0000494 & No & $1 / 9(11 \%)$ \\
\hline Upslanted palpebral fissure & HP:0000582 & No & $1 / 9(11 \%)$ \\
\hline Short nose & HP:0003196 & No & $1 / 9(11 \%)$ \\
\hline Depressed nasal bridge & HP:0005280 & Yes & - \\
\hline Bulbous nasal tip & HP:0000414 & Yes & - \\
\hline Anteverted nares & HP:0000463 & Yes & $1 / 9(11 \%)$ \\
\hline Thin upper lip vermilion & HP:0000219 & Yes & - \\
\hline Macroglossia & HP:0000158 & No & $1 / 9(11 \%)$ \\
\hline Preauricular pit & HP:0004467 & No & $1 / 9(11 \%)$ \\
\hline Dysplastic ears & HP:0000377 & Yes & - \\
\hline Microtia & HP:0008551 & No & $1 / 9(11 \%)$ \\
\hline \multicolumn{4}{|l|}{ Neurologic } \\
\hline Intellectual disability & HP:0001249 & No & $3 / 9(33 \%)$ \\
\hline Developmental delay & HP:0001263 & Yes & $6 / 9(67 \%)$ \\
\hline Speech delay & HP:0000750 & Yes & $8 / 9(89 \%)$ \\
\hline Speech apraxia & HP:0011098 & Yes & $3 / 9(33 \%)$ \\
\hline Motor delay & HP:0001270 & No & $6 / 9(67 \%)$ \\
\hline Motor dyspraxia & HP:0004302 & Yes & $3 / 9(33 \%)$ \\
\hline \multirow{2}{*}{$\begin{array}{l}\text { Autism Spectrum Disorder (ASD) } \\
\text { Attention Deficit Hyperactivity Disorder } \\
\text { (ADHD) }\end{array}$} & HP:0000729 & No & $5 / 9(56 \%)$ \\
\hline & HP:0007018 & Yes & $4 / 9(44 \%)$ \\
\hline \multirow{2}{*}{$\begin{array}{l}\text { Encopresis } \\
\text { Hypertonicity }\end{array}$} & HP:0040183 & No & $1 / 9(11 \%)$ \\
\hline & HP:0001276 & No & $1 / 9(11 \%)$ \\
\hline $\begin{array}{l}\text { Hypersensitive sensory processing } \\
\text { deficit }\end{array}$ & HP:0003474 & No & $1 / 9(11 \%)$ \\
\hline Congenital torticollis & HP:0005988 & No & $1 / 9(11 \%)$ \\
\hline Myoclonic jerks & HP:0001336 & No & $1 / 9(11 \%)$ \\
\hline Sleep disturbance & HP:0002360 & No & $1 / 9(11 \%)$ \\
\hline \multicolumn{4}{|l|}{ Eyes } \\
\hline & HP:0000483 & No & $2 / 9(22 \%)$ \\
\hline \multicolumn{4}{|l|}{ Otolaryngology } \\
\hline \multicolumn{4}{|l|}{ Choanal atresia } \\
\hline High palate & HP:0000218 & No & $3 / 9(33 \%)$ \\
\hline \multicolumn{4}{|l|}{ Cardiovascular } \\
\hline \multicolumn{4}{|l|}{ Atrial septal defect } \\
\hline Pulmonary artery stenosis & HP:0004415 & Yes & $1 / 9(11 \%)$ \\
\hline Patent Foramen Ovale (PFO) & HP:0001655 & Yes & - \\
\hline \multicolumn{3}{|l|}{ Respiratory } & \\
\hline Asthma & HP:0002099 & No & $1 / 9(11 \%)$ \\
\hline \multicolumn{4}{|l|}{ Hands/Toes Anomalies } \\
\hline Brachydactyly & HP:0001156 & No & $1 / 9(11 \%)$ \\
\hline Short phalanx of finger & HP:0009803 & No & $1 / 9(11 \%)$ \\
\hline Short toe & HP:0001831 & No & $1 / 9(11 \%)$ \\
\hline Other & & & \\
\hline
\end{tabular}




\begin{tabular}{|c|c|c|c|}
\hline $\begin{array}{l}\text { Specialized schooling required } \\
\text { Speech therapy }\end{array}$ & & $\begin{array}{l}\text { Yes } \\
\text { Yes }\end{array}$ & $1 / 9(11 \%)$ \\
\hline \multicolumn{4}{|l|}{ MRI } \\
\hline \multirow{5}{*}{$\begin{array}{l}\text { Enlarged ventricles } \\
\text { Thin corpus callosum } \\
\text { Dysgenesis of corpus callosum } \\
\text { Hyperintensity in left periventricular } \\
\text { white matter } \\
\text { Absent splenium }\end{array}$} & \multirow{5}{*}{$\begin{array}{l}\text { HP:0002079 } \\
\text { HP:0006989 }\end{array}$} & Unknown & 1/9 (11\%) \\
\hline & & Unknown & $3 / 9(33 \%)$ \\
\hline & & Unknown & $1 / 9(11 \%)$ \\
\hline & & Unknown & $1 / 9(11 \%)$ \\
\hline & & Unknown & $1 / 9(11 \%)$ \\
\hline
\end{tabular}

Clinical phenotypes of patients with $A N K S 1 B$ haploinsufficiency syndrome are according to Carbonell et al. (2019). 
Supplementary Table 4 Comparison of clinical features between the DGRC0030 - t(1;3) proband and those with pathogenic SNVs and 1q41q42 microdeletions either encompassing WDR26 or not encompassing WDR26

\begin{tabular}{|c|c|c|c|c|c|}
\hline \multirow{3}{*}{$\begin{array}{c}\text { DGRC0030 - t(1;3) } \\
\text { proband's phenotype }\end{array}$} & \multirow{3}{*}{$\begin{array}{c}\text { HPO } \\
\text { nomenclature }\end{array}$} & \multirow{3}{*}{$\begin{array}{c}\text { Pathogenic WDR26 } \\
\text { SNVs } \mathrm{n}=15(\%)\end{array}$} & \multicolumn{3}{|c|}{ 1q41q42 microdeletions } \\
\hline & & & \multicolumn{2}{|c|}{ encompassing WDR26 } & \multirow{2}{*}{$\begin{array}{c}\text { proximal to WDR26 } \\
\mathrm{n}=8(\%)\end{array}$} \\
\hline & & & $<4 \mathrm{Mb} \mathrm{n}=12(\%)$ & $>4 \mathrm{Mb} \mathrm{n}=13(\%)$ & \\
\hline Neurologic & & $15(100)$ & $12(100)$ & $11(85)$ & $7(88)$ \\
\hline \multicolumn{6}{|l|}{ Central Nervous System } \\
\hline $\begin{array}{l}\text { Severe global developmental delay with } \\
\text { enuresis and encopresis }\end{array}$ & $H P: 0011344$ & $15(100)$ & $11(92)$ & $9(69)$ & $6(75)$ \\
\hline Delayed speech and language development & HP:0000750 & $11(73)$ & $7(58)$ & $1(8)$ & $1(13)$ \\
\hline Absent speech & HP:0001344 & $2(13)$ & $1(8)$ & $1(8)$ & 0 \\
\hline Broad based ataxic gait & HP:0002136 & $9(60)$ & $1(8)$ & $1(8)$ & $1(13)$ \\
\hline Hypotonia & $H P: 0001290$ & $9(60)$ & 4 (33) & $3(23)$ & $3(38)$ \\
\hline Feeding problems & HP:0011968 & $4(27)$ & $2(17)$ & $2(15)$ & $1(13)$ \\
\hline Seizures $^{\mathrm{a}}$ & (HP:0001250) & $15(100)$ & $9(75)$ & $6(46)$ & $2(25)$ \\
\hline Abnormality of brain morphologyb & (HP:0012443) & $10(67)$ & $8(67)$ & $9(69)$ & $1(13)$ \\
\hline \multicolumn{6}{|l|}{ Behavioral psychiatric manifestations } \\
\hline Behavioral problems & HP:0000708 & $4(27)$ & $2(17)$ & 0 & $2(25)$ \\
\hline Autistic features & HP:0000729 & $5(33)$ & $1(8)$ & 0 & 0 \\
\hline Happy and/or friendly personality & HP:0040082 & $10(67)$ & $3(25)$ & 0 & 0 \\
\hline Craniofacial & & $15(100)$ & $12(100)$ & $11(85)$ & $4(50)$ \\
\hline Mild microcephaly & HP:0040196 & $11(73)$ & $2(17)$ & $4(31)$ & $2(25)$ \\
\hline Full cheeks as a child & - & $11(73)$ & $1(8)$ & $1(8)$ & 0 \\
\hline Coarse facial features & HP:0000280 & $12(80)$ & $6(50)$ & $4(31)$ & 0 \\
\hline Prominent maxilla & $H P: 0430028$ & $13(87)$ & $2(17)$ & 0 & 0 \\
\hline Depressed nasal bridge & $H P: 0005280$ & $5(33)$ & $5(38)$ & $5(45)$ & $2(25)$ \\
\hline Anteverted nares & HP:0000463 & $8(53)$ & $2(17)$ & $2(15)$ & 0 \\
\hline Full/broad nasal tip & HP:0000453 & $11(73)$ & $5(42)$ & $3(23)$ & $1(13)$ \\
\hline Wide mouth & HP:0000154 & $4(17)$ & $2(17)$ & $1(8)$ & 0 \\
\hline Decreased cupid's bow & HP:0010800 & $11(73)$ & $3(25)$ & $1(8)$ & 0 \\
\hline Tented upper lip vermilion & HP:0010804 & 0 & $4(33)$ & $2(15)$ & $2(25)$ \\
\hline Protruding upper lip & $H P: 0000215$ & $13(87)$ & $5(42)$ & $3(23)$ & $3(38)$ \\
\hline Widely spaced teeth & HP:0000687 & $13(87)$ & $5(42)$ & $2(15)$ & 0 \\
\hline Growth & & $12(80)$ & $6(50)$ & $4(31)$ & $3(38)$ \\
\hline Postnatal growth retardation & HP:0008897 & $11(73)$ & $6(50)$ & $4(31)$ & $3(38)$ \\
\hline Eyes & & $1(7)$ & $2(17)$ & $4(31)$ & $2(25)$ \\
\hline Strabismus & HP:0000486 & $1(7)$ & $1(8)$ & $2(15)$ & $2(25)$ \\
\hline Cardiovascular (Congenital heart disease) & $H P: 0030680$ & $2(13)$ & $3(25)$ & $2(15)$ & $0(0)$ \\
\hline $\begin{array}{l}\text { Ventricular septal defect - Subaortic } \\
\text { interventricular communication }\end{array}$ & $\begin{array}{l}\text { HP:0001629- } \\
\text { HP:0011681 }\end{array}$ & $1(7)$ & 0 & $2(15)$ & 0 \\
\hline Tetralogy of Fallot & $H P: 0001636$ & 0 & 0 & 0 & 0 \\
\hline Right Aortic Arch & HP:0012020 & $1(7)$ & 0 & 0 & 0 \\
\hline
\end{tabular}




\begin{tabular}{|c|c|c|c|c|c|}
\hline Genitourinary (Male genital anomalies) & HP:0000032 & $1(7)$ & $3(25)$ & $3(23)$ & $1(13)$ \\
\hline Phimosis & HP:0001741 & 0 & 0 & $3(23)$ & 0 \\
\hline Hydrocele testis & HP:0000034 & 0 & 0 & 0 & 0 \\
\hline Midline defect & & $1(7)$ & $6(50)$ & $7(54)$ & $0(0)$ \\
\hline Umbilical hernia & HP:0001537 & 0 & 0 & 0 & 0 \\
\hline Chest & & $0(0)$ & $1(8)$ & $0(0)$ & $0(0)$ \\
\hline Accessory or supernumerary nipple & HP:0002558 & 0 & $1(8)$ & 0 & 0 \\
\hline Skeletal & & $4(27)$ & $3(25)$ & $4(31)$ & $0(0)$ \\
\hline Thoracic scoliosis & HP:0002943 & $2(13)$ & $1(8)$ & 0 & 0 \\
\hline Clubfoot or bilateral talipes equinovarus & $\mathrm{HP}: 0001776$ & 0 & $2(17)$ & $4(31)$ & 0 \\
\hline Posture & & $1(7)$ & $0(0)$ & $1(8)$ & $0(0)$ \\
\hline Neck hyperextension & & $1(7)$ & 0 & $1(8)$ & 0 \\
\hline
\end{tabular}

Shaded in light gray are clinical features of the DGRC0025-t(1;3) proband predominantly shared with subjects with pathogenic SNVs and 1q41q42 microdeletions either encompassing WDR26 or not, whereas those in dark gray are predominantly shared by subjects with affected WDR26. Pathogenic WDR26 SNVs as well as 1q41q42 microdeletions are mainly from Skraban et al. (2017). aHave not been reported yet; bHave not been identified by magnetic resonance imaging. 
Supplementary Table 5 liGS libraries and mapping metrics of the analyzed cases

\begin{tabular}{|c|c|c|c|c|c|c|c|c|c|c|c|c|}
\hline Metrics & \multicolumn{2}{|c|}{$\begin{array}{c}\text { DGRC0016 } \\
t(16 ; 17)\end{array}$} & \multicolumn{2}{|c|}{$\begin{array}{c}\text { DGRC0019 } \\
t(2 ; 19)\end{array}$} & \multicolumn{2}{|c|}{$\begin{array}{c}\text { DGRC0006 } \\
t(8 ; 14)\end{array}$} & \multicolumn{2}{|c|}{$\begin{array}{l}\text { DGRC0013 } \\
\text { inv(13) }\end{array}$} & \multicolumn{2}{|c|}{$\begin{array}{c}\text { DGRC0025 } \\
\text { t(12;17) }\end{array}$} & \multicolumn{2}{|c|}{$\begin{array}{c}\text { DGRC0030 } \\
t(1 ; 3)\end{array}$} \\
\hline \multicolumn{13}{|l|}{ liGS libraries } \\
\hline Reads per library & $146.11 \mathrm{M}$ & - & $196.26 \mathrm{M}$ & - & $165.53 \mathrm{M}$ & - & $129.11 \mathrm{M}$ & - & $212.96 \mathrm{M}$ & - & $208.62 \mathrm{M}$ & - \\
\hline Read-pairs per library & $73.07 \mathrm{M}$ & - & $98.13 \mathrm{M}$ & - & $82.77 \mathrm{M}$ & - & $64.56 \mathrm{M}$ & - & $106.48 \mathrm{M}$ & - & $104.31 \mathrm{M}$ & - \\
\hline Median insert size (bp) & 2,877 & - & 3,110 & - & 3,326 & - & 3,429 & - & 3,188 & - & 3,136 & - \\
\hline Sequence coverage & 1.02 & - & 1.26 & - & 1.09 & - & 0.84 & - & 1.5 & - & 1.16 & - \\
\hline \multicolumn{13}{|l|}{ Mapping } \\
\hline Total mapped reads & $142.22 \mathrm{M}$ & 97.34 & $182.89 \mathrm{M}$ & 93.70 & $162.55 \mathrm{M}$ & 98.2 & $127.06 \mathrm{M}$ & 98.41 & $207.66 \mathrm{M}$ & 97.51 & $202.08 \mathrm{M}$ & 96.86 \\
\hline Singletons ${ }^{b}$ & $2.92 \mathrm{M}$ & 2.17 & $2.45 \mathrm{M}$ & 2.19 & $2.48 \mathrm{M}$ & 1.5 & $1.88 \mathrm{M}$ & 1.46 & $4.49 \mathrm{M}$ & 2.11 & $5.51 \mathrm{M}$ & 2.64 \\
\hline Total mapped read-pairs & $69.42 \mathrm{M}$ & 95.02 & $89.53 \mathrm{M}$ & 91.24 & $80.04 \mathrm{M}$ & 96.7 & $62.59 \mathrm{M}$ & 96.95 & $101.58 \mathrm{M}$ & 95.40 & $98.29 \mathrm{M}$ & 94.22 \\
\hline Duplicated mapped read-pairs & $5.63 \mathrm{M}$ & 8.24 & $42.09 \mathrm{M}$ & 47.10 & $4.37 \mathrm{M}$ & 5.27 & $3.23 \mathrm{M}$ & 5.00 & $6.44 \mathrm{M}$ & 6.05 & 7.07M & 6.78 \\
\hline Distinctly mapped read-pairs & $63.79 \mathrm{M}$ & 94.61 & $47.45 \mathrm{M}$ & 84.66 & $75.93 \mathrm{M}$ & 91.74 & $59.54 \mathrm{M}$ & 92.24 & $95.65 \mathrm{M}$ & 89.83 & $80.16 \mathrm{M}$ & 76.84 \\
\hline
\end{tabular}

aedian Absolute Deviation; ${ }^{\mathrm{b}}$ Reads mapped without a pair 
Supplementary Table 6 Summary of interchr chimeric and intrachr inv type structural variants identified at different resolution

\begin{tabular}{|c|c|c|c|c|c|c|c|c|c|c|c|c|}
\hline \multirow[t]{2}{*}{ Analysis } & \multicolumn{2}{|c|}{$\begin{array}{c}\text { DGRC0016 } \\
t(16 ; 17)\end{array}$} & \multicolumn{2}{|c|}{$\begin{array}{c}\text { DGRC0019 } \\
t(2 ; 19)\end{array}$} & \multicolumn{2}{|c|}{$\begin{array}{c}\text { DGRC0006 } \\
t(8 ; 14)\end{array}$} & \multicolumn{2}{|c|}{$\begin{array}{c}\text { DGRC0013 } \\
\text { inv(13) }\end{array}$} & \multicolumn{2}{|c|}{$\begin{array}{c}\text { DGRC0025 } \\
\text { t(12;17) }\end{array}$} & \multicolumn{2}{|c|}{$\begin{array}{c}\text { DGRC0030 } \\
t(1 ; 3)\end{array}$} \\
\hline & Chimeric & Improper & Chimeric & Improper & Chimeric & Improper & Chimeric & Improper & Chimeric & Improper & Chimeric & Improper \\
\hline \multicolumn{13}{|l|}{ Identification method } \\
\hline Vumber of read-pair clusters ${ }^{a}$ & 375 & 135 & 481 & 111 & 550 & 186 & 378 & 130 & 515 & 182 & 477 & 176 \\
\hline Blacklist filtering ${ }^{b}$ & 269 & 108 & 312 & 84 & 309 & 149 & 214 & 112 & 292 & 148 & 290 & 139 \\
\hline $\begin{array}{l}\text { Sub-clusteringc } \\
\text { (Cplt Clu; Incplt Clu) }\end{array}$ & $\begin{array}{l}62 \\
207\end{array}$ & $38 ; 70$ & $78 ; 234$ & $26 ; 58$ & $124 ; 185$ & $76 ; 73$ & $76 ; 138$ & $59 ; 53$ & $138 ; 154$ & $95 ; 33$ & $109 ; 181$ & $76 ; 63$ \\
\hline $\begin{array}{l}<3 \text { read-pairs filterd } \\
\text { (Cplt Clu; Incplt Clu) }\end{array}$ & $22 ; 93$ & - & $16 ; 74$ & - & $26 ; 59$ & - & $18 ; 54$ & - & $25 ; 44$ & - & $21 ; 57$ & - \\
\hline \multicolumn{13}{|c|}{ Structural variants - fully;partly resolved ${ }^{\mathrm{e}}$} \\
\hline Translocation & $1 ; ; 0$ & - & $1^{\mathrm{f}} ; 0$ & - & $1^{\mathrm{f}} ; 0$ & - & $0 ; 0$ & - & $1^{\mathrm{f}} ; 0$ & - & $1^{\mathrm{f}} ; 0$ & - \\
\hline omal insertion & $11 ; 8$ & - & $6 ; 8$ & - & $18 ; 8$ & - & $10 ; 9$ & - & $13 ; 10$ & - & $8 ; 10$ & - \\
\hline Invers & - & $29 ; 17$ & - & $20 ; 19$ & - & $49 ; 9$ & - & $42^{f} ; 10$ & - & $61 ; 7$ & - & $49 ; 7$ \\
\hline | insertion & - & $6 ; 11$ & - & $3 ; 10$ & - & $15 ; 11$ & - & $12 ; 11$ & - & $25 ; 3$ & - & $21 ; 6$ \\
\hline Complex SV & & $5 ; 4$ & - & $5 ; 0$ & - & $8 ; 2$ & - & $8 ; 1$ & - & $5 ; 2$ & - & $8 ; 1$ \\
\hline Total & & 40 & 35 & & \multicolumn{2}{|c|}{$91 ; 30$} & \multicolumn{2}{|c|}{$72 ; 31$} & \multicolumn{2}{|c|}{$105 ; 22$} & \multicolumn{2}{|c|}{$87 ; 24$} \\
\hline \multicolumn{5}{|c|}{ At clinical resolution of $\geq 10 \mathrm{~kb}-$ fully;partly resolved ${ }^{9}$} & & & & & & & & \\
\hline Interchromosomal insertion & $1 ; 0$ & - & $1 ; 0$ & - & $3 ; 0$ & - & $1(1) ; 0$ & - & $0 ; 0$ & - & $0 ; 0$ & - \\
\hline Inversion & - & $2 ; 3$ & - & $1 ; 3$ & - & $4(1) ; 2$ & - & $7^{\dagger}(1) ; 2$ & - & $7 ; 0$ & - & $3(1) ; 1$ \\
\hline mosomal insertion & - & $0 ; 1$ & - & $0 ; 2$ & - & $1 ; 0$ & - & $1 ; 0$ & - & $3 ; 0$ & - & $0 ; 0$ \\
\hline Complex SV & ${ }_{7(2}$ & $\begin{array}{l}4(2) ; 2 \\
; 6\end{array}$ & $-6(4)$ & $\begin{array}{l}4(4) ; 0 \\
5\end{array}$ & - & $\begin{array}{l}3(3) ; 2(1) \\
4(1)\end{array}$ & $\overline{12}_{12}$ & $\begin{array}{l}3(2) ; 1(1) \\
; 3(1)\end{array}$ & $-13(1)$ & $\begin{array}{l}3(1) ; 2(1) \\
; 2(1)\end{array}$ & - $6(3)$; & $\begin{array}{l}3(2) ; 1(1) \\
2(1)\end{array}$ \\
\hline \multicolumn{5}{|c|}{ At liGS resolution of $<10 \mathrm{~kb}-$ fully;partly resolved ${ }^{\mathrm{g}}$} & & & & & & & & \\
\hline Interchromosomal insertion & $10 ; 8$ & - & $5 ; 8$ & - & $15(2) ; 8(1)$ & - & $9 ; 9(1)$ & - & $13(2) ; 10$ & - & $8 ; 10$ & - \\
\hline Inversion & - & $27 ; 14$ & - & $19 ; 16$ & - & $45(2) ; 7$ & - & $35 ; 8$ & - & $55(10) ; 7$ & - & $46(3) ; 6$ \\
\hline Intrachromosomal insertion & - & $6 ; 10$ & - & $3 ; 8$ & - & $14 ; 11$ & - & $11(1) ; 11$ & - & $22(4) ; 3$ & - & $21(3) ; 6$ \\
\hline Complex SV & - & $1 ; 2$ & - & $1(1) ; 0$ & - & $5(1) ; 0$ & - & $5(2) ; 0$ & - & $2 ; 0$ & - & $5(1) ; 0$ \\
\hline Total & 44 & 34 & $28(1)$ & 32 & 79(5); & $26(1)$ & $60(3)$ & $28(1)$ & $92(16$ & ); 20 & $80(7$ & $; 22$ \\
\hline
\end{tabular}

Read-pairs are considered interchr or chimeric if both reads of the pair map to different chromosomes, allowing identification of translocations and interchr ins. On the other hand, those considered intrachr inv type improper read-pairs map on the same chromosome and in the same orientation, allowing identification of inv, intrachr ins and cx variants. Clusters of different categories (complete and incomplete) as well as fully

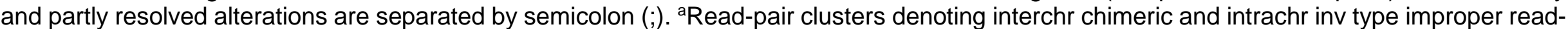
pair clusters were identified by an in-house developed Python script improperCLAS.py and clustered by readPairCluster (Talkowski et al. 2011). 'Genomic regions delimited by different type of clusters were filtered against the regions of the "blacklist", with an overlap cutoff of $\geq 30 \%$. 'If possible, according to orientation of each read of each cluster specific read-pair, a cluster is divided into two breakpoint specific sub-clusters 
constituting a complete cluster (Cplt Clu) or otherwise they are considered as incomplete clusters (Incplt Clu). dSub-clusters with less than 3 read-

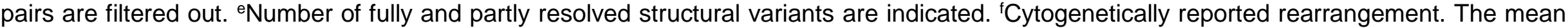
number of read-pairs that define breakpoints of cytogenetically reported rearrangement was 24 (ranging 14 to 43 ) whereas breakpoint resolution was about $200 \mathrm{bp}$ (ranging from zero to 919). 9Proband-specific alterations and those with a frequency lower than $1 \%$ in the SVref dataset are presented in parentheses. The SVref dataset is according to Collins et al. (2017). Patient specific alterations are also presented in Supplementary Table 15. 
Supplementary Table 7 del and dup identified by different methods, cross validations, at different resolutions and frequencies

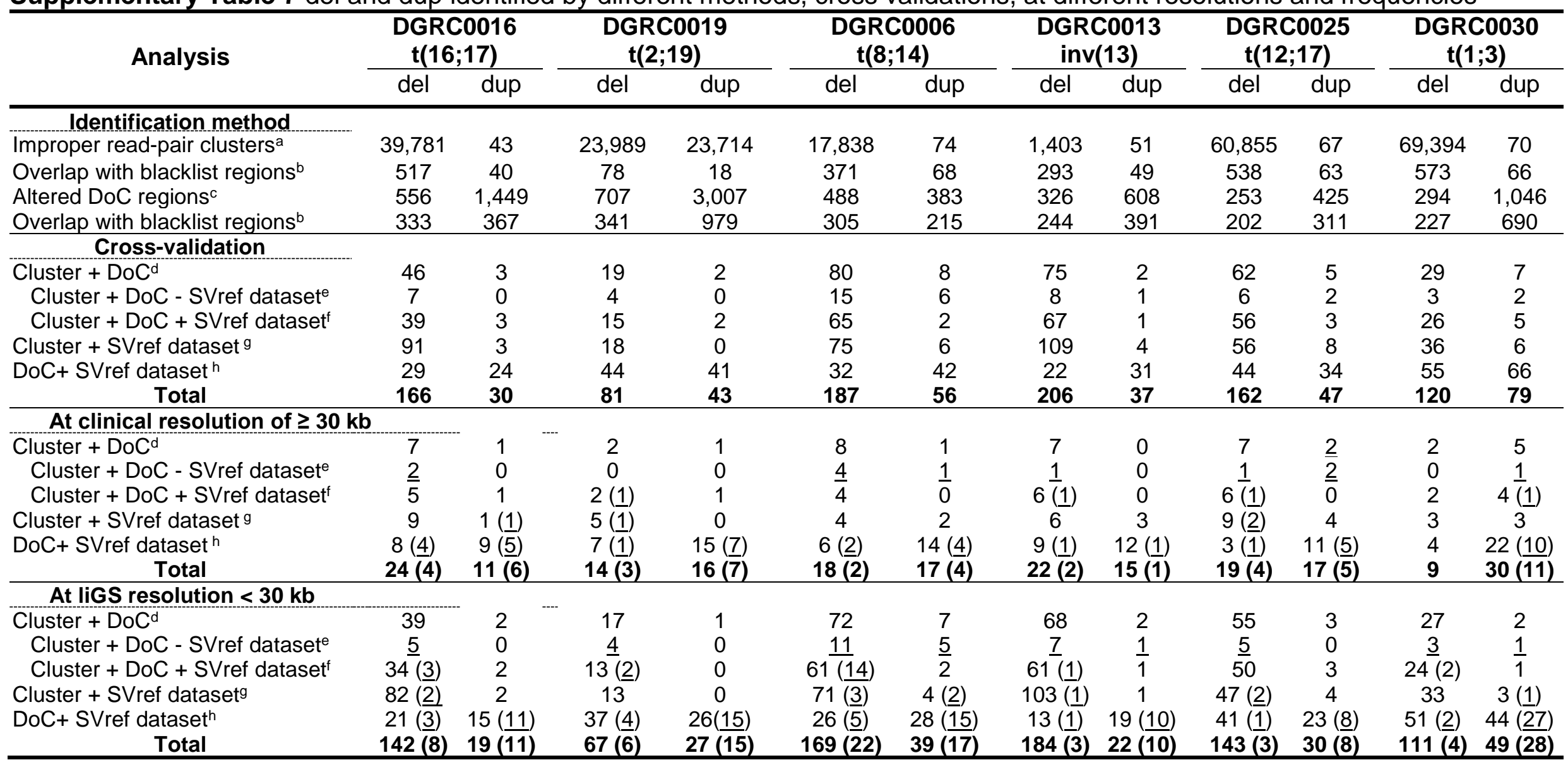

Total number of CNVs identified by each analysis are shown. Identified CNVs validated in the SVref dataset with a frequency $<1 \%$ are in parentheses. The underlined numbers indicate CNVs analyzed in more detail using the CNV-ConTool.

almproper read-pairs denoting del and tandem dup were identified by an in-house developed Python script designated improperCLAS.py and clustered together by readPairCluster (Talkowski et al. 2011). Inward and outward facing read-pairs with insert size (IS) larger than the median IS $+3^{*}$ IS SD denote improper del and dup read-pairs, respectively. ${ }^{b}$ Genomic regions delimited by read-pair clustering and DoC analysis were filtered against $\geq 30 \%$ overlap with the genomic regions defined in the "blacklist". "Identification of del and dup through DoC analysis, performed by CN.MOPS. ${ }^{d}$ Total number of del and dup identified by read-pair clustering and DoC analysis. ${ }^{e}$ del and dup identified by read-pair clustering 
and DoC analysis, not reported in the SVref dataset. ${ }^{f}$ del and dup identified by read-pair clustering and DoC analysis, that are reported in the

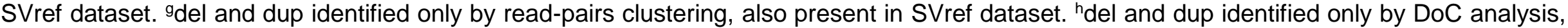
also present in SVref dataset. 
Supplementary Table 8 Protein coding and non-coding RNA genes localized within hESC TADs disrupted by DGRC0016 t(16;17) breakpoints and associated phenotypes

\begin{tabular}{|c|c|c|c|c|c|c|c|c|c|}
\hline \multicolumn{4}{|c|}{ Genes } & \multicolumn{6}{|c|}{ OMIM and DD2GP based pathologies } \\
\hline GeneCard & OMIM & oe $(90 \% \mathrm{Cl})$ & $\% \mathrm{HI}$ & Clinical phenotype & OMIM & Inheritance & $\begin{array}{l}\text { DD2GP } \\
\text { category }\end{array}$ & \multicolumn{2}{|c|}{ PMID reference } \\
\hline \multicolumn{10}{|c|}{ 16q24.3 breakpoint within hESC TAD at 16:89246091-89686091 } \\
\hline ANKRD11 - IVS2 & 611192 & $0.05(0.02-0.11)$ & 75.55 & KBG syndrome & 148050 & $A D$ & confirmed & 15378538 & 21782149 \\
\hline \multicolumn{10}{|c|}{ der(16) g.[chr16:pter_cen_89401716_89401717del::chr17:46784035_qter] } \\
\hline SPG7 & 602783 & 1.26 & 61.82 & Spastic paraplegia 7, AR & 607259 & $A D ; A R^{a}$ & nd & na & na \\
\hline RPL13 & 113703 & $0.00(0.00-0.32)$ & 58.62 & - & na & nd & nd & na & na \\
\hline CPNE7 & 605689 & 1.05 & 75.88 & - & na & nd & nd & na & na \\
\hline DPEP1 & 179780 & 0.85 & 77.20 & - & na & nd & nd & na & na \\
\hline CHMP1A & 164010 & 0.61 & 33.54 & Pontocerebellar hypoplasia, type 8 & 614961 & AR & probable & 23023333 & na \\
\hline SPATA33 & 615409 & 1.51 & 98.71 & - & na & nd & nd & na & na \\
\hline & & & & Al Kaissi syndrome & 617694 & AR & nd & na & na \\
\hline CDK10 & 603464 & 1.01 & 51.33 & $\begin{array}{l}\text { Severe Growth Retardation Spine Malformations } \\
\text { and Developmental Delays }\end{array}$ & & & possible & 28886341 & 29130579 \\
\hline \multicolumn{10}{|c|}{ 17q21.31 breakpoint within hESC TAD at 17:46687454-47647635 } \\
\hline NSF & 601633 & $0.33(0.18-0.61)$ & 4.09 & - & na & nd & nd & na & na \\
\hline WNT3 - IVS3 & 165330 & $0.13(0.05-0.39)$ & 13.89 & Tetra-amelia syndrome 1 & 273395 & AR & confirmed & na & na \\
\hline \multicolumn{10}{|c|}{ der(17) g.[chr17:pter_cen_46781999_46784034del::chr16:89401718_qter] } \\
\hline WNT9B & 602864 & $0.20(0.08-0.62)$ & 34.84 & - & na & nd & nd & na & na \\
\hline GOSR2 & 604027 & 0.82 & 32.66 & Epilepsy, progressive myoclonic 6 & 614018 & AR & nd & na & na \\
\hline$C D C 27$ & 116946 & $0.12(0.06-0.25)$ & 9.78 & - & na & nd & nd & na & na \\
\hline \multirow[t]{3}{*}{ MYL4 } & 160770 & 0.74 & 34.00 & Atrial fibrillation, familial, 18 & 617280 & $A D$ & nd & na & na \\
\hline & & & & Bleeding disorder, platelet-type, 16, AD & 187800 & $A D$ & nd & na & na \\
\hline & & & & Glanzmann thrombasthenia & 273800 & AR & nd & na & na \\
\hline \multirow[t]{3}{*}{ ITGB3 } & 173470 & $0.32(0.20-0.51)$ & 35.78 & Purpura, posttransfusion & na & nd & nd & na & na \\
\hline & & & & Thrombocytopenia, neonatal alloimmune & na & nd & nd & na & na \\
\hline & & & & Myocardial infarction, susceptibility to & 608446 & nd & nd & na & na \\
\hline NPEPPS & 606793 & $0.00(0.00-0.06)$ & 11.57 & - & na & nd & nd & na & na \\
\hline
\end{tabular}

Analysis performed with bioinformatics tool TAD-GConTool. Reference genome assembly is GRCh38/hg38, and human embryonic stem cells (hESC) TADs are according to Dixon et al. (2012). Genes showing high probability of being disease causing are in bold (McKusick 1998; Wright et al. 2015). LoF intolerance, is expressed as observed / expected number (oe) of LoF variants. For genes with oe $<0.35$ the oe at $90 \% \mathrm{Cl}$ is also stated. HI, Haploinsufficiency index; na, not assigned; nd, not determined; AR, Autosomal Recessive, AD, Autosomal Dominant. ${ }^{2} \mathrm{~A}$ dominant effect for some SPG7 mutations has been reported (Sánchez-Ferrero et al. 2013). 
Supplementary Table 9 Protein coding and non-coding RNA genes localized within hESC TADs disrupted by DGRC0019 t(2;19) breakpoints and associated phenotypes

\begin{tabular}{|c|c|c|c|c|c|c|c|c|c|}
\hline \multicolumn{4}{|c|}{ Genes } & \multicolumn{6}{|c|}{ OMIM and DD2GP based pathologies } \\
\hline GeneCard & OMIM & oe $(90 \% \mathrm{Cl})$ & $\% \mathrm{HI}$ & Clinical phenotype & OMIM & Inheritance & $\begin{array}{l}\text { DD2GP } \\
\text { category }\end{array}$ & \multicolumn{2}{|c|}{ PMID reference } \\
\hline \multicolumn{10}{|c|}{ 2p13.3 breakpoint within hESC TAD at 2:70279364-70999362 } \\
\hline PCYOX1 & 610995 & 1.00 & 55.49 & - & na & nd & nd & na & na \\
\hline SNRPG & 603542 & $0.00(0.00-0.55)$ & 7.48 & - & na & nd & nd & na & na \\
\hline FAM136A & 616275 & 0.93 & 74.63 & - & na & nd & nd & na & na \\
\hline TGFA & 190170 & $0.11(0.04-0.55)$ & 2.83 & - & na & nd & nd & na & na \\
\hline$A D D 2$ & 102681 & $0.13(0.07-0.27)$ & 37.61 & - & na & nd & nd & na & na \\
\hline FIGLA & 608697 & 0.65 & 65.57 & Premature ovarian failure 6 & 612310 & $A D$ & nd & na & na \\
\hline CLEC4F & na & 0.91 & 90.63 & - & na & nd & nd & na & na \\
\hline CD207 & 604862 & 0.84 & 86.68 & Birbeck granule deficiency & 613393 & nd & nd & na & na \\
\hline LINC01143 & na & nd & nd & - & na & nd & nd & na & na \\
\hline VAX2 & 604295 & $0.33(0.14-1.04)$ & 47.75 & - & na & nd & nd & na & na \\
\hline ATP6V1B1 - IVS1 & 192132 & $0.63(0.43-0.93)$ & 46.96 & Renal tubular acidosis with deafness & 267300 & AR & confirmed & 12566520 & 9916796 \\
\hline AC007040 - Exon 5 & na & nd & nd & - & na & nd & nd & na & na \\
\hline \multicolumn{10}{|c|}{ der(2) g.[chr19:32878515_qterinv::chr2:70941503_70941506del_cen_qter] } \\
\hline ANKRD53 & 617009 & 0.83 & 89.63 & - & na & nd & nd & na & na \\
\hline TEX261 & na & 0.59 & 28.75 & - & na & nd & nd & na & na \\
\hline \multicolumn{10}{|c|}{ 19q13.11 breakpoint within hESC TAD at 19:32577254-33217254 } \\
\hline PDCD5 & 604583 & 0.56 & 14.97 & - & na & nd & nd & na & na \\
\hline ANKRD27 & na & 0.83 & 48.32 & - & na & nd & nd & na & na \\
\hline RGS9BP & 607814 & 0.86 & 74.66 & Bradyopsia & 608415 & nd & nd & na & na \\
\hline AC008736 & na & nd & nd & - & na & nd & nd & na & na \\
\hline NUDT19 & na & 1.96 & 87.63 & - & na & nd & nd & na & na \\
\hline TDRD12 & na & 0.98 & 59.89 & - & na & nd & nd & na & na \\
\hline SLC7A9 & 604144 & 0.75 & 61.03 & Cystinuria & 220100 & $A D ; A R$ & nd & na & na \\
\hline CEP89 - Exon 18 & 615470 & $0.93(0.72-1.21)$ & 82.86 & - & na & nd & nd & na & na \\
\hline \multicolumn{10}{|c|}{ der(19) g.[chr19:pter_cen_32878513_32878514del::CATA::chr2:pter_70941502inv] } \\
\hline FAAP24 & 610884 & nd & nd & - & na & nd & nd & na & na \\
\hline RHPN2 & 617932 & 0.49 & 64.24 & - & na & nd & nd & na & na \\
\hline GPATCH1 & na & 0.48 & 58.94 & - & na & nd & nd & na & na \\
\hline
\end{tabular}




\begin{tabular}{|c|c|c|c|c|c|c|c|c|}
\hline WDR88 & na & 0.73 & 83.65 & - & na & nd & nd & na \\
\hline$L R P 3$ & 603159 & 0.47 & 60.40 & - & na & nd & nd & na \\
\hline SLC7A10 & 607959 & 0.47 & 40.99 & - & na & nd & nd & na \\
\hline
\end{tabular}

Analysis performed with bioinformatics tool TAD-GConTool. Reference genome assembly is GRCh38/hg38, and human embryonic stem cells (hESC) TADs are according to Dixon et al. (2012). oe, LoF intolerance, expressed as observed / expected number of LoF variants. For genes with oe $<0.35$ the oe at $90 \% \mathrm{Cl}$ is also stated. HI, Haploinsufficiency index; na, not assigned; nd, not determined; AR, Autosomal Recessive; AD, Autosomal Dominant. 
Supplementary Table 10 Protein coding and non-coding RNA genes localized within hESC TADs disrupted by DGRC0019 t(2;19) breakpoints with their associated phenotypes and GWAS data from the referred regions

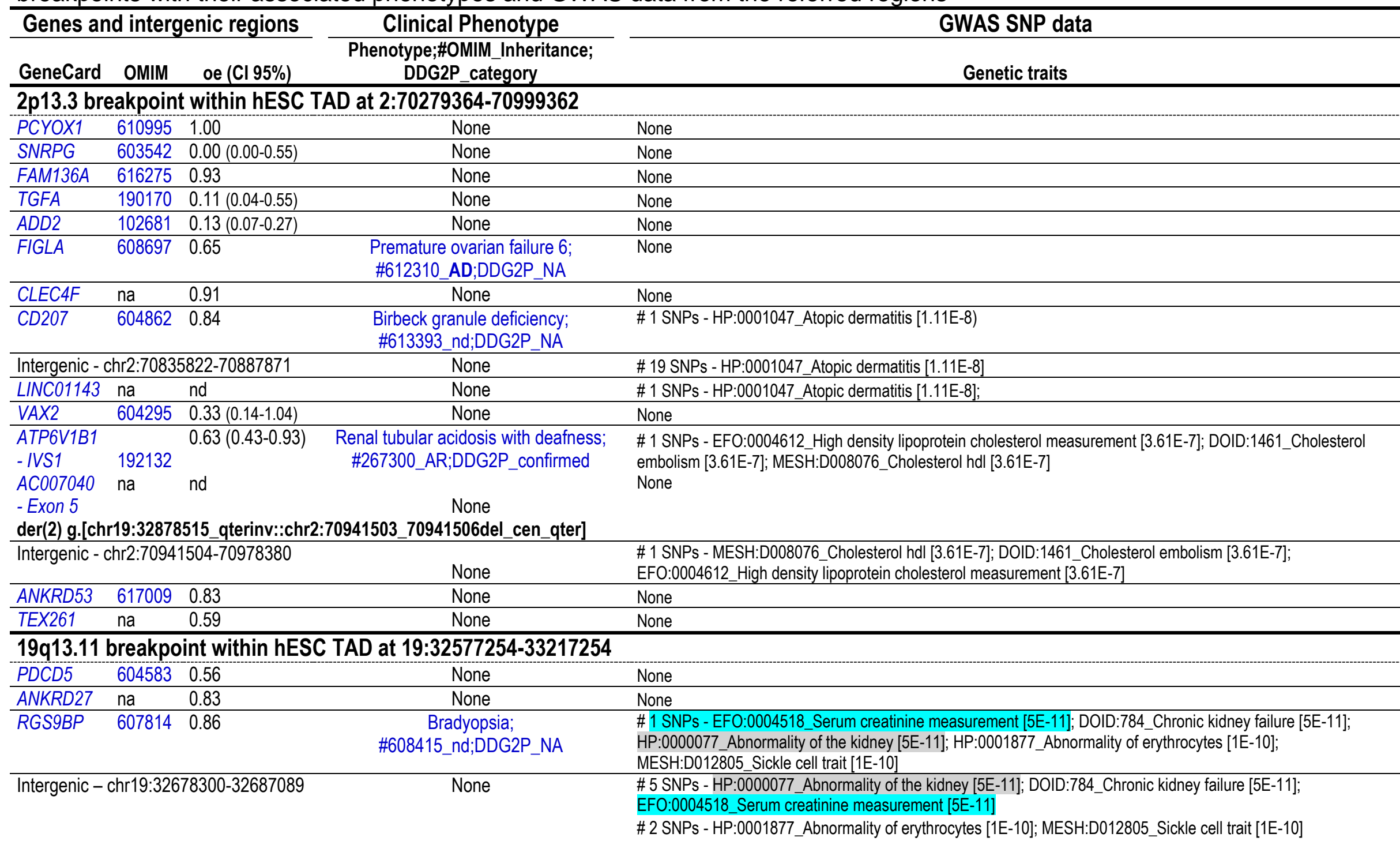




\begin{tabular}{|c|c|c|}
\hline AC008736 na & None & $\begin{array}{l}\text { \# } 3 \text { SNPs - HP:0001877_Abnormality of erythrocytes [1E-10]; MESH:D012805_Sickle cell trait [1E-10] } \\
\text { \# } 1 \text { SNPs - EFO:0004518_Serum creatinine measurement [5E-11]; DOID:784_Chronic kidney failure [5E-11]; } \\
\text { HP:0000077_Abnormality of the kidney [5E-11] }\end{array}$ \\
\hline Intergenic - chr19:32691750-32691961 & None & \# 1 SNPs - HP:0001877_Abnormality of erythrocytes [1E-10]; MESH:D012805_Sickle cell trait [1E-10] \\
\hline $\begin{array}{lll}\text { NUDT19 } & \text { na } & 1.96\end{array}$ & None & $\begin{array}{l}\text { \# } 10 \text { SNPs - HP:0001877_Abnormality of erythrocytes [1E-10]; MESH:D012805_Sickle cell trait [1E-10] } \\
\text { \# } 3 \text { SNPs - EFO:0004518_Serum creatinine measurement [5E-11]; DOID:784_Chronic kidney failure [5E-11]; } \\
\text { HP:0000077_Abnormality of the kidney [5E-11] }\end{array}$ \\
\hline Intergenic - chr19:32713796-32719753 & None & $\begin{array}{l}\text { \# } 4 \text { SNPs - HP:0001877_Abnormality of erythrocytes [1E-10]; MESH:D012805_Sickle cell trait [1E-10] } \\
\text { \# } 1 \text { SNPs - HP:0000077_Abnormality of the kidney [5E-11]; DOID:784_Chronic kidney failure [5E-11]; } \\
\text { EFO:0004518_Serum creatinine measurement [5E-11] }\end{array}$ \\
\hline TDRD12 & None & $\begin{array}{l}\text { \# } 23 \text { SNPs - EFO:0004518_Serum creatinine measurement [5E-11]; DOID:784_Chronic kidney failure [5E-11]; } \\
\text { HP:0000077_Abnormality of the kidney [5E-11] } \\
\text { \# } 15 \text { SNPs - HP:0001877_Abnormality of erythrocytes [1E-10]; MESH:D012805_Sickle cell trait [1E-10] }\end{array}$ \\
\hline Intergenic - chr19:32829580-32830509 & None & $\begin{array}{l}\text { \# 1 SNPs - DOID:614_Lymphopenia [5.85E-8]; EFO:0004518_Serum creatinine measurement [5E-11]; } \\
\text { DOID:784_Chronic kidney failure [5E-11]; HP:0000077_Abnormality of the kidney [5E-11] }\end{array}$ \\
\hline $\begin{array}{lll}\text { SLC7A9 } & 604144 \quad 0.75\end{array}$ & $\begin{array}{l}\text { Cystinuria; \#220100 } \\
\text { AD,AR;DDG2P_NA }\end{array}$ & $\begin{array}{l}\text { \# } 32 \text { SNPs - DOID:784_Chronic kidney failure [5E-11]; HP:0000077_Abnormality of the kidney [5E-11] } \\
\text { \# } 7 \text { SNPs - DOID:655_Inherited metabolic disorder [1E-16]; HP:0001939_Abnormality of metabolism homeostasis } \\
\text { [1E-16] } \\
\text { \# } 4 \text { SNPs - HP:0001877_Abnormality of erythrocytes [1E-10]; MESH:D012805_Sickle cell trait [1E-10] }\end{array}$ \\
\hline Intergenic - chr19:32869766-32875925 & None & $\begin{array}{l}\text { \# } 7 \text { SNPs - DOID:655_Inherited metabolic disorder [1E-16]; HP:0001939_Abnormality of metabolism homeostasis } \\
\text { [1E-16] } \\
\text { \# } 5 \text { SNPs - DOID:784_Chronic kidney failure [5E-11]; HP:0000077_Abnormality of the kidney [5E-11] } \\
\text { \# } 3 \text { SNPs - EFO:0004518_Serum creatinine measurement [5E-11] }\end{array}$ \\
\hline der(19) g.[chr19:pter_cen_32878513_3 & ::CATA::chr2:pter_7 & $\begin{array}{l}\text { \# } 109 \text { SNPs - DOID:2841_Asthma [5.85E-8]; DOID:1240_Leukemia [5.85E-8]; DOID:614_Lymphopenia [5.85E-8]; } \\
\text { HP:0002665_Lymphoma [5.85E-8] } \\
\text { \# } 66 \text { SNPs - DOID:784_Chronic kidney failure [5E-11]; HP:0000077_Abnormality of the kidney [5E-11] } \\
\text { \# } 62 \text { SNPs - EFO:0004518_Serum creatinine measurement [5E-11] } \\
\text { \# } 7 \text { SNPs - DOID:655_Inherited metabolic disorder [1E-16]; HP:0001939_Abnormality of metabolism homeostasis } \\
\text { [1E-16] } \\
\text { \# } 1 \text { SNPs - HP:0001370_Rheumatoid arthritis [1.76E-7] } \\
\text { nv] }\end{array}$ \\
\hline Intergenic - chr19:32878514-32972209 & None & $\begin{array}{l}\text { \# } 106 \text { SNPs - DOID:614_Lymphopenia [5.85E-8]; DOID:2841_Asthma [5.85E-8]; HP:0002665_Lymphoma [5.85E- } \\
\text { 8]; DOID:1240_Leukemia [5.85E-8] } \\
\text { \# } 63 \text { SNPs - DOID:784_Chronic kidney failure [5E-11]; HP:0000077_Abnormality of the kidney [5E-11] } \\
\text { \# } 60 \text { SNPs - EFO:0004518_Serum creatinine measurement [5E-11] } \\
\text { \# } 6 \text { SNPs - DOID:655_Inherited metabolic disorder [1E-16]; HP:0001939_Abnormality of metabolism homeostasis } \\
\text { [1E-16] } \\
\text { \# } 1 \text { SNPs - HP:0001370_Rheumatoid arthritis [1.76E-7] }\end{array}$ \\
\hline 610884 nd & None & $\begin{array}{l}\text { \# } 3 \text { SNPs - EFO:0004518_Serum creatinine measurement [5E-11]; DOID:784_Chronic kidney failure [5E-11]; } \\
\text { HP:0000077_Abnormality of the kidney [5E-11] }\end{array}$ \\
\hline
\end{tabular}




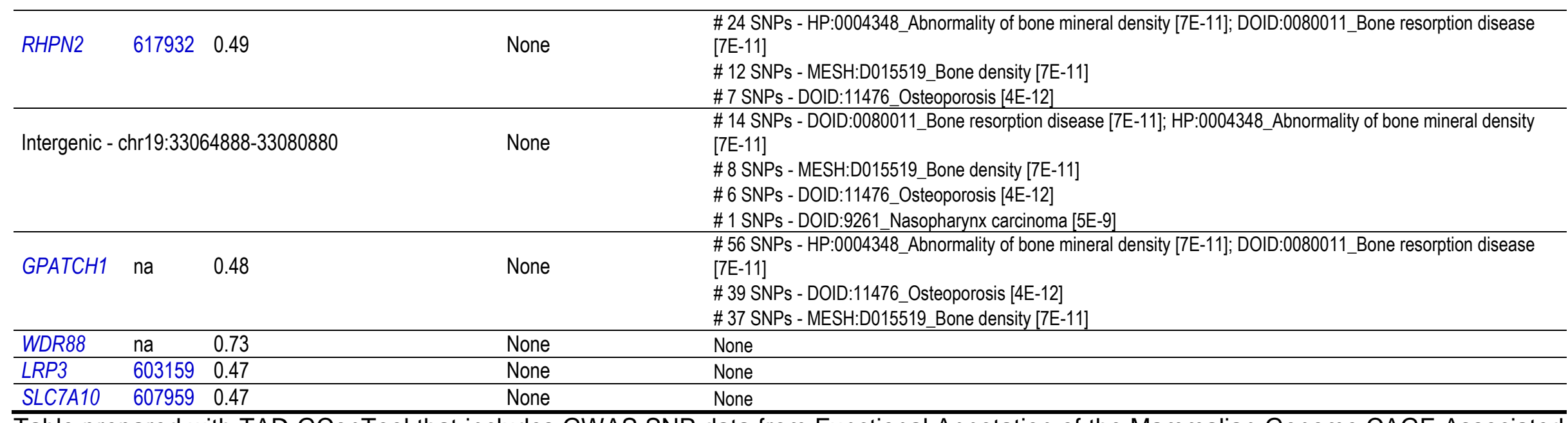

Table prepared with TAD-GConTool that includes GWAS SNP data from Functional Annotation of the Mammalian Genome CAGE Associated Transcriptome (FANTOM CAT) database ((Hon et al. 2017). The SNP associated genetic traits are presented according to their ontology term ID. Although the conventional GWAS significance threshold is $\leq 5.00 \mathrm{E}-8$, for disrupted genes, adjacent genomic regions and for genetic traits identified in GWAS overlapping the proband's clinical phenotype, a threshold of 9.99E-6 is used. As an example, genetic traits clearly associated with this locus are highlighted in different colors. Clinical phenotype data are from OMIM and Developmental Disorders Genotype-Phenotype (DDG2P) databases. The clinical phenotype data are configured as follows: i) gene-associated phenotypes in humans from either database; ii) \#OMIM phenotype number underscore type of inheritance (AR, AD or AR/AD) or \#NA in the absence of OMIM phenotype number; and DDG2P underscore phenotype classification (confirmed, probable and possible) or DDG2P_NA in the absence of DDG2P data. 
Supplementary Table 11 del and dup identified in DGRC0016 t(16;17) by read-pair clustering and DoC analysis and cross validated using these data and an SVref dataset

\begin{tabular}{|c|c|c|c|c|c|c|c|}
\hline \multirow{2}{*}{$\begin{array}{l}\text { Cluster/Coverage/ } \\
\text { Array/Collins2017_IDa }\end{array}$} & \multirow[b]{2}{*}{ Genomic variants [GRCh38] $^{\mathrm{b}}$} & \multirow[b]{2}{*}{$\begin{array}{l}\text { Size } \\
(\mathbf{k b})\end{array}$} & \multirow{2}{*}{$\begin{array}{c}\text { Ref.. } \\
\text { group }\end{array}$} & \multirow{2}{*}{$\begin{array}{l}\text { OMIM gene } \\
\text { or } \\
\text { phenotype }^{\mathrm{d}}\end{array}$} & \multicolumn{2}{|c|}{ Genomic variants } & \multirow{2}{*}{$\begin{array}{l}\text { ACMG } \\
\text { classif. }^{h}\end{array}$} \\
\hline & & & & & $\begin{array}{c}<1 \% \\
\text { Frequency }{ }^{\mathrm{e}, \mathrm{f}, \mathrm{g}} \\
\end{array}$ & $\begin{array}{c}\text { PMID } \\
\text { reference }\end{array}$ & \\
\hline & At clinical resolution of $\geq 30 \mathbf{k b}$ ( $<1 \%$ frequency) & & & & & & \\
\hline $\begin{array}{c}\text { Coverage } \\
\text { Collins2017_DUP_3 }\end{array}$ & 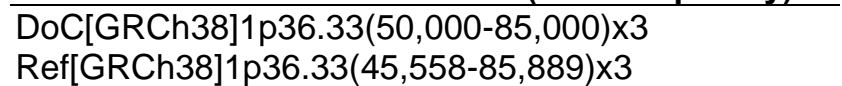 & $\begin{array}{l}35.00 \\
40.33\end{array}$ & - & - & $\frac{\mathrm{nsv} 544867}{(0.01)^{f}}$ & $\underline{21841781}$ & VUS \\
\hline Cluster (29) & Clu[GRCh38] 3p24.1(27,354,585-27,408,246) & 53.66 & & & & & \\
\hline Coverage & DoC[GRCh38] 3p24.1(27,360,001-27,410,000)x1 & 50.00 & - & SLC4A7 & Familial & - & VUS \\
\hline Sanger validated & SanS[GRCh38] chr3:g.27,354,680_27,408,191del & 53.51 & & & & & \\
\hline Cluster (21) & Clu[GRCh38] 8q24.21(129,061,012-129,897,302) & 836.29 & & CCDC26, & & & \\
\hline Coverage/Array & DoC[GRCh38] 8q24.21(129,060,001-129,900,000)x1 & 840.00 & - & GSDMC, & de novo & - & VUS \\
\hline Sanger validated & SanS[GRCh38] chr8:g.129061233_129897281del & 836.05 & & & & & \\
\hline $\begin{array}{c}\text { Coverage } \\
\text { Collins2017_DEL_3501 }\end{array}$ & $\begin{array}{l}\text { DoC[GRCh38]9q13(62,400,000-62,580,000)x1 } \\
\text { Ref[GRCh38]9q13(62,368,699-62,550,699)x1 }\end{array}$ & $\begin{array}{l}180.00 \\
182.00\end{array}$ & - & - & $(0.87)^{g}$ & $\underline{28260531}$ & VUS \\
\hline $\begin{array}{c}\text { Coverage } \\
\text { Collins2017_DEL_3519 }\end{array}$ & $\begin{array}{l}\text { DoC[GRCh38] 9q13q21.1 }(64,940,000-65,010,000) \times 1 \\
\text { Ref[GRCh38] 9q13q21.1 }(64,937,582-64,998,124) \times 1\end{array}$ & $\begin{array}{l}70.00 \\
60.54\end{array}$ & - & - & $(0.29)^{g}$ & $\underline{28260531}$ & VUS \\
\hline $\begin{array}{c}\text { Coverage } \\
\text { Collins2017_DEL_4027 }\end{array}$ & 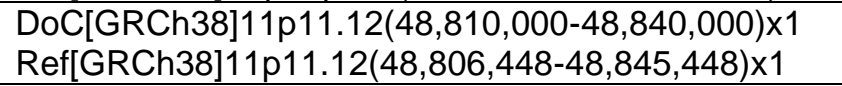 & $\begin{array}{l}30.00 \\
39.00\end{array}$ & - & - & $\frac{\text { esv2677500 }}{(0.09)^{\mathrm{e}}}$ & $\underline{23128226}$ & VUS \\
\hline $\begin{array}{c}\text { Coverage } \\
\text { Collins2017_DEL_6280 }\end{array}$ & $\begin{array}{l}\text { DoC[GRCh38]Xq21.31(90,195,000-90,240,000)x1 } \\
\text { Ref[GRCh38]Xq21.31(90,192,814-90,241,972)x1 }\end{array}$ & $\begin{array}{l}45.00 \\
49.16 \\
\end{array}$ & - & - & $\frac{\text { esv2676290 }}{(0.17)^{\mathrm{e}}}$ & $\underline{23128226}$ & VUS \\
\hline \multicolumn{8}{|c|}{ 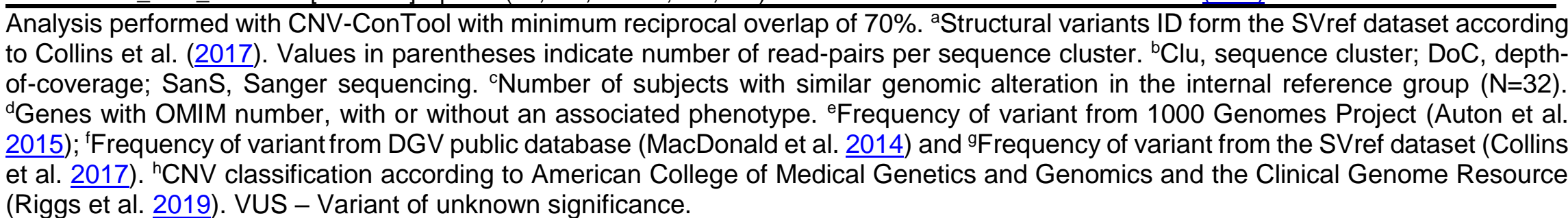 } \\
\hline
\end{tabular}


Supplementary Table 12 Protein coding and non-coding RNA genes localized within the 836.05 kb del at 8q24.21, and GWAS data from the referred region

\begin{tabular}{|c|c|c|c|c|}
\hline \multicolumn{3}{|c|}{ Genes and intergenic regions } & \multirow{2}{*}{$\begin{array}{l}\text { Clinical phenotype } \\
\text { OMIM\# Phenotype } \\
\text { Inheritance; (DDG2P) }\end{array}$} & \multirow{2}{*}{$\begin{array}{c}\text { GWAS SNP data } \\
\text { Genetic traits }\end{array}$} \\
\hline GeneCard & OMIM & oe (Cl 95\%) & & \\
\hline \multicolumn{5}{|c|}{ 8q24.21 breakpoint within hESC TAD at 8:126838573-129758572 } \\
\hline \multicolumn{5}{|c|}{ chr8: g.129061233_129897281del } \\
\hline LINC00977 & na & nd & None & $\begin{array}{l}\text { \# } 7 \text { SNPs - HP:0000202_Oral cleft [7.03E-10]; EFO:0003959_Progranulin measurement [7.03E-10]; MESH:D002971_Cleft } \\
\text { lip [7.03E-10]; DOID:0050567_Orofacial cleft [7.03E-10] }\end{array}$ \\
\hline CCDC26 & na & nd & None & $\begin{array}{l}\text { \# } 43 \text { SNPs - HP:0003002_Breast carcinoma [4.79E-10]; HP:0100615_Ovarian neoplasm [4.79E-10] } \\
\text { \# } 42 \text { SNPs - EFO:0005090_Monocyte count [3.00E-20] } \\
\text { \# } 29 \text { SNPs - MESH:D007962_Leukocytes [2.00E-10] } \\
\text { \# } 22 \text { SNPs - HP:0004386_Gastrointestinal inflammation [2.00E-9]; DOID:0050589_Inflammatory bowel disease [2.00E-9] }\end{array}$ \\
\hline \multicolumn{3}{|c|}{ Intergenic - chr8:129061233-129685401 } & None & $\begin{array}{l}\text { \# } 130 \text { SNPs - HP:0000202_Oral cleft [7.03E-10]; EFO:0003959_Progranulin measurement [7.03E-10]; } \\
\text { MESH:D002971_Cleft lip [7.03E-10]; DOID:0050567_Orofacial cleft [7.03E-10] } \\
\text { \# } 109 \text { SNPs - MESH:D005910_Glioma [5E-21]; DOID:0060108_Brain glioma [5E-21]; EFO:0000326_Central nervous } \\
\text { system cancer [5E-21] } \\
\text { \# } 42 \text { SNPs - EFO:0005090_Monocyte count [3E-20] } \\
\text { \# } 34 \text { SNPs - HP:0003002_Breast carcinoma [4.79E-10]; HP:0100615_Ovarian neoplasm [4.79E-10] } \\
\text { \# } 29 \text { SNPs - MESH:D007962_Leukocytes [2E-10] } \\
\text { \# } 25 \text { SNPs - DOID:9261_Nasopharynx carcinoma [2E-18] } \\
\text { \# } 22 \text { SNPs - DOID:0050589_Inflammatory bowel disease [2E-9]; HP:0004386_Gastrointestinal inflammation [2E-9] }\end{array}$ \\
\hline GSDMC & 608384 & 0.86 & None & $\begin{array}{l}\text { \# 1 SNPs - MESH:D005910_Glioma [5.00E-21]; EFO:0000326_Central nervous system cancer [5.00E-21]; } \\
\text { DOID:0060108_Brain glioma [5.00E-21]; DOID:9261_Nasopharynx carcinoma [2.00E-18]; }\end{array}$ \\
\hline \multicolumn{5}{|c|}{ 8q24.21 breakpoint within hESC TAD at 8:129798572-130718572 } \\
\hline \multicolumn{3}{|c|}{ Intergenic - chr8:129798572-129839593 } & None & None \\
\hline FAM49B - IVS1 & na & $0.10(0.04-0.31)$ & None & None \\
\hline
\end{tabular}

Table performed by TAD-GConTool, that includes GWAS SNP data from FANTOM CAT database (Hon et al. 2017), with GWAS significance threshold at $\leq 5.00 \mathrm{E}-8$. The GWAS associated traits are presented according to their ontology term ID. Clinical phenotypes associated with genes were obtain from both OMIM and DDG2P databases. 
Supplementary Table 13 del and dup identified in DGRC0019 t(2;19) by read-pair clustering and DoC analysis and cross validated using these data and an SVref dataset

\begin{tabular}{|c|c|c|c|c|c|c|c|}
\hline \multirow{2}{*}{$\begin{array}{l}\text { Cluster/Coverage/ } \\
\text { Array/Collins2017_ID }\end{array}$} & \multirow[b]{2}{*}{ Genomic variants [GRCh38] ${ }^{\mathrm{b}}$} & \multirow{2}{*}{$\begin{array}{l}\text { Size } \\
(\mathbf{k b})\end{array}$} & \multirow{2}{*}{$\begin{array}{l}\text { Ref. } \\
\text { groupc }\end{array}$} & \multirow{2}{*}{$\begin{array}{l}\text { OMIM gene } \\
\text { or } \\
\text { phenotype }^{\mathrm{d}}\end{array}$} & \multicolumn{2}{|c|}{ Genomic variants } & \multirow{2}{*}{$\begin{array}{l}\text { ACMG } \\
\text { classif. }^{g}\end{array}$} \\
\hline & & & & & $\begin{array}{c}<1 \% \\
\text { Frequency } \\
\end{array}$ & $\begin{array}{l}\text { PMID } \\
\text { reference }\end{array}$ & \\
\hline & At clinical resolution of $\geq 30 \mathbf{k b}$ ( $<1 \%$ frequency) & & & & & & \\
\hline $\begin{array}{c}\text { Coverage } \\
\text { Collins2017_DUP } 1803\end{array}$ & $\begin{array}{l}\text { DoC[GRCh38]10q11.22(47,560,000-47,600,000)x3 } \\
\text { Ref[GRCh38]10q11.22(47,560,011-47,592,990)x3 }\end{array}$ & $\begin{array}{l}40.00 \\
32.98\end{array}$ & - & - & $(0.15)^{f}$ & $\underline{28260531}$ & VUS \\
\hline $\begin{array}{c}\text { Coverage } \\
\text { Collins2017_DUP } 1917\end{array}$ & 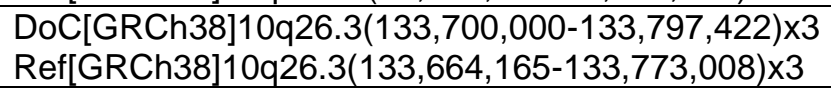 & $\begin{array}{c}97.42 \\
108.84\end{array}$ & - & - & $(0.73)^{f}$ & $\underline{28260531}$ & VUS \\
\hline $\begin{array}{c}\text { Coverage } \\
\text { Collins2017_DUP_2593 }\end{array}$ & $\begin{array}{l}\text { DoC[GRCh38]16p11.2(32,850,000-32,880,000)x3 } \\
\text { Ref[GRCh38]16p11.2(32,859,125-32,883,735)×3 }\end{array}$ & $\begin{array}{l}30.00 \\
24.61\end{array}$ & - & - & $(0.15)^{f}$ & $\underline{28260531}$ & VUS \\
\hline $\begin{array}{c}\text { Cluster (32) } \\
\text { Coverage/Array } \\
\text { Collins2017_DEL_5923 }\end{array}$ & 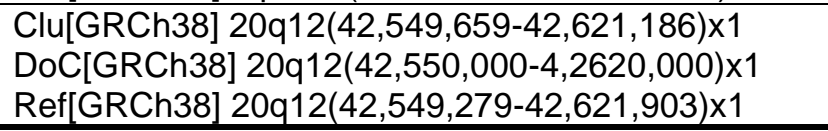 & $\begin{array}{l}71.53 \\
70.00 \\
72.62 \\
\end{array}$ & - & PTPRT & $\frac{\text { nsv519654 }}{{\underline{(0.25)^{e}}}^{\mathrm{e}}}$ & $\underline{19592680}$ & VUS \\
\hline $\begin{array}{c}\text { Coverage } \\
\text { Collins2017_DUP_124 }\end{array}$ & 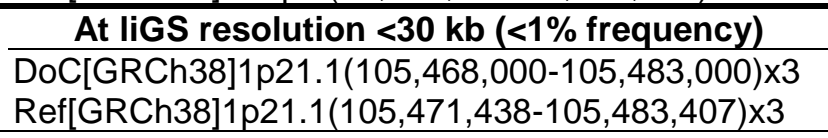 & $\begin{array}{l}15.00 \\
11.97 \\
\end{array}$ & & - & $(0.44)^{f}$ & $\underline{28260531}$ & VUS \\
\hline $\begin{array}{c}\text { Coverage } \\
\text { Collins2017 DUP } 1350\end{array}$ & $\begin{array}{l}\text { DoC[GRCh38]7q36.1 }(150,945,000-150,960,000) \times 3 \\
\text { Ref[GRCh38]7q36.1(150,946,081-150,959,579)x3 }\end{array}$ & $\begin{array}{l}15.00 \\
13.49\end{array}$ & - & KCNH2 & $(0.15)^{f}$ & $\underline{28260531}$ & VUS \\
\hline $\begin{array}{c}\text { Cluster(5) } \\
\text { Coverage } \\
\text { Collins2017_DEL_2724 }\end{array}$ & $\begin{array}{l}\text { Clu[GRCh38]7p12.2(49875020-49885550)x1 } \\
\text { DoC[GRCh38]7p12.2(49,875,000-49,888,000)x1 } \\
\text { Ref[GRCh38]7p12.2(49,873,862-49,886,430)x1 }\end{array}$ & $\begin{array}{l}10.53 \\
13.00 \\
12.57 \\
\end{array}$ & - & VWC2 & $(0.29)^{f}$ & $\underline{28260531}$ & VUS \\
\hline $\begin{array}{c}\text { Coverage } \\
\text { Collins2017_DUP } 1318\end{array}$ & $\begin{array}{l}\text { DoC[GRCh38]7q31.1(109,797,000-109,812,000)x3 } \\
\text { Ref[GRCh38]7q31.1 }(109,794,416-109,815,260) \times 3\end{array}$ & $\begin{array}{l}15.00 \\
20.85\end{array}$ & 1 & - & $(0.87)^{f}$ & $\underline{28260531}$ & VUS \\
\hline $\begin{array}{c}\text { Coverage } \\
\text { Collins2017_DUP_1972 }\end{array}$ & $\begin{array}{l}\text { DoC[GRCh38]11p14.1(28,983,000-28,995,000)x3 } \\
\text { Ref[GRCh38]11p14.1(28,982,519-28,994,389)x3 }\end{array}$ & $\begin{array}{l}12.00 \\
11.87\end{array}$ & 3 & - & $(0.15)^{f}$ & 28260531 & VUS \\
\hline $\begin{array}{c}\text { Coverage } \\
\text { Collins2017_DUP_1984 }\end{array}$ & 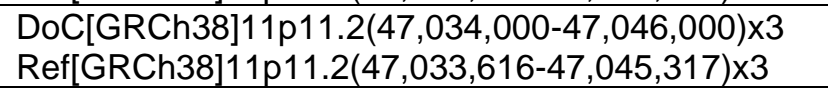 & $\begin{array}{l}12.00 \\
11.70\end{array}$ & 7 & - & $(0.44)^{f}$ & 28260531 & VUS \\
\hline $\begin{array}{l}\text { Cluster }(8) \\
\text { Coverage }\end{array}$ & 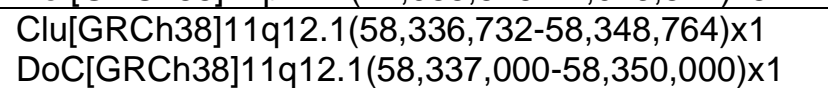 & $\begin{array}{l}12.03 \\
13.00\end{array}$ & & - & novel & - & VUS \\
\hline
\end{tabular}


Coverage

DoC[GRCh38]19q13.43(56,960,000-56,970,000)x1

10.00

10.17

-

-

esvis

esv3556681

23714750

VUS

Analysis performed with CNV-ConTool with minimum reciprocal overlap of $70 \%$. aStructural variants ID form the SVref dataset is according to Collins et al. (2017); Values in parentheses indicate number of read-pairs per sequence cluster. bClu, sequence cluster; DoC, depth-of-coverage. ' Number of subjects with similar genomic alteration in the internal reference group ( $\mathrm{N}=32$ ). ${ }^{\mathrm{d}} \mathrm{Genes}$ with $\mathrm{OMIM}$ number, with or without an associated phenotype. eFrequency of variant from DGV public database (MacDonald et al. 2014) and ${ }^{\mathrm{f}}$ Frequency of variant from SVref dataset (Collins et al, 2017). ${ }^{9}$ CNV classification according to American College of Medical Genetics and Genomics and the Clinical Genome Resource (Riggs et al. 2019). VUS - Variant of unknown significance. 
Supplementary Table 14 Cross-validated, probands'-specific del and dup identified in the retrospectively analyzed subjects at both resolutions

\begin{tabular}{|c|c|c|c|c|}
\hline Cluster/ Coverage/Array ${ }^{a}$ & Genomic variants [GRCh38] ${ }^{b}$ & Size (kb) & OMIM gene or phenotype ${ }^{c}$ & $\begin{array}{c}\text { ACMG } \\
\text { Classification }^{d}\end{array}$ \\
\hline \multicolumn{5}{|c|}{ At clinical resolution of $\geq 30 \mathrm{~kb}$} \\
\hline & DGRC0006 - t(8;14) & & \multirow{3}{*}{-} & \multirow{3}{*}{ VUS } \\
\hline Cluster (5) & Clu[GRCh38]12q23.3(105,626,312-105,679,417)x1 & 53.11 & & \\
\hline Coverage & DoC[GRCh38]12q23.3(105,627,000-105,680,000)x1 & 53.00 & & \\
\hline & DGRC0013 - inv(13) & & \multirow[b]{3}{*}{-} & \multirow{3}{*}{ VUS } \\
\hline Cluster (24) & Clu[GRCh38]7q36.1(148,576,804-148,659,035)x1 & 82.23 & & \\
\hline Coverage/Array & DoC[GRCh38]7q36.1 $(148,577,000-148,660,000) \times 1$ & 83.00 & & \\
\hline & DGRC0030 - t(1;3) & & \multirow{3}{*}{ PEX14 } & \multirow[b]{3}{*}{ VUS } \\
\hline Cluster (31) & Clu[GRCh38]1p36.22(10,478,274-10,524,390)x3 & 46.12 & & \\
\hline Coverage & DoC[GRCh38]1p36.22(10,480,000-10,521,000)x3 & 41.00 & & \\
\hline \multicolumn{5}{|c|}{ At liGS resolution $<30 \mathrm{~kb}$} \\
\hline & DGRC0006 - t(8;14) & & \multirow{3}{*}{ - } & \multirow{3}{*}{ VUS } \\
\hline Cluster (32) & Clu[GRCh38]2p23.1 $(30,166,431-30,183,788) \times 1$ & 17.36 & & \\
\hline Coverage & DoC[GRCh38]2p23.1(30,166,000-30,184,000)x1 & 18.00 & & \\
\hline Cluster (26) & Clu[GRCh38]Xq27.2(141,880,897-141,895,782)x3 & 14.89 & \multirow{2}{*}{ MAGEC3 } & \multirow{2}{*}{ VUS } \\
\hline \multirow[t]{2}{*}{ Coverage } & DoC[GRCh38]Xq27.2 $(141,884,000-141,894,000) \times 3$ & 10.00 & & \\
\hline & DGRC0013 - inv(13) & & \multirow{3}{*}{-} & \multirow{3}{*}{ VUS } \\
\hline Cluster(9) & Clu[GRCh38]4q28.2(128,771,573-128,784,988)×3 & 13.42 & & \\
\hline Coverage & DoC[GRCh38]4q28.2(128,772,000-128,784,000)x3 & 12.00 & & \\
\hline
\end{tabular}

Analysis performed with the reported workflow and CNV-ConTool with minimum reciprocal overlap of $70 \%$. ${ }^{\text {Values in parentheses indicate }}$ number of read-pairs per sequence cluster. ${ }^{b} \mathrm{Clu}$, sequence cluster; DoC, Depth-of-Coverage. ${ }^{\mathrm{C}}$ Genes with OMIM number, with or without an associated phenotype. ${ }^{d}$ CNV classification according to American College of Medical Genetics and Genomics and the Clinical Genome Resource (Riggs et al. 2019). VUS - Variant of unknown significance. 
Supplementary Table 15 Probands'-specific inv, ins and cx variants identified in retrospectively analyzed subjects

\begin{tabular}{|c|c|c|c|c|c|}
\hline Alteration & Cluster $^{\mathrm{a}}$ & Genomic variants [GRCh38] ${ }^{b}$ & Size (kb) & $\begin{array}{l}\text { OMIM gene or } \\
\text { phenotype }^{c}\end{array}$ & $\begin{array}{l}\text { Other genes and } \\
\text { non coding } \\
\text { RNAs }\end{array}$ \\
\hline \multicolumn{6}{|c|}{ DGRC0006 - t(8;14) } \\
\hline inv Fully Rslv ${ }^{d}$ & Cluster (30) & Clu[GRCh38]4p13(43,736,343-43,752,624)inv & 16.28 & - & - \\
\hline inv Fully Rslv & Cluster (20) & Clu[GRCh38]1p13.2(112,873,541-112,878,455)inv & 4.91 & - & $R P 3-522 D 1.1$ \\
\hline $\begin{array}{l}\text { interchr ins } \\
\text { Fully Rslv }\end{array}$ & Cluster (6) & $\begin{array}{l}\text { Excision: Clu[GRCh38]8q22.3(100,715,571-100,717,804) } \\
\text { Insertion: Clu[GRCh38]3q25.31(155,308,345-155,310,600) }\end{array}$ & 2.23 & $\begin{array}{c}P A B P C 1 \\
-\end{array}$ & - \\
\hline $\begin{array}{l}\text { dellNVdel } \\
\text { Fully Rslv }\end{array}$ & Cluster (6) & $\begin{array}{l}\text { Clu[GRCh38]6q16.3(100,113,153-100,115,762)del } \\
\text { Clu[GRCh38]6q16.3(100,115,763-100,115,785)inv } \\
\text { Clu[GRCh38]6q16.3(100,115,786-100,119,075)del } \\
\end{array}$ & 5.92 & - & - \\
\hline \multicolumn{6}{|c|}{ DGRC0013 - inv(13) } \\
\hline $\begin{array}{l}\text { interchr ins } \\
\text { Fully Rslv }\end{array}$ & Cluster (13) & $\begin{array}{l}\text { Excision: Clu[GRCh38]9q34.11(129,424,605-129,444,016) } \\
\text { Insertion: Clu[GRCh38]22q11.23(23,510,309-23,510,596) }\end{array}$ & 19.41 & 一 & $R P 3-65 J 3$ \\
\hline $\begin{array}{l}\text { interchr. ins } \\
\text { Partly Rslv }\end{array}$ & Cluster (24) & $\begin{array}{l}\text { Excision: Clu[GRCh38]2q31.2(177,984,262-?) } \\
\text { Insertion: Clu[GRCh38]12q13.2(55,390,573-?) }\end{array}$ & - & PDE11A & - \\
\hline $\begin{array}{l}\text { dellNVdel } \\
\text { Fully Rslv }\end{array}$ & Cluster (3) & $\begin{array}{l}\text { Clu[GRCh38]12q13.2(49,784,688-49,788,877)del } \\
\text { Clu[GRCh38]12q13.2(49,788,877-49,788,985)inv } \\
\text { Clu[GRCh38]12q13.2(49,788,986-49,792,873)del } \\
\end{array}$ & 8.18 & NCKAP5L & - \\
\hline $\begin{array}{l}\text { dellNVdel } \\
\text { Fully Rslv }\end{array}$ & Cluster (4) & $\begin{array}{l}\text { Clu[GRCh38]13q12.11(22,446,323-22,449,632)del } \\
\text { Clu[GRCh38]13q12.11(22,449,633-22,449,661)inv } \\
\text { Clu[GRCh38]13q12.11(22,449,662-22,452,615)del } \\
\end{array}$ & 6.29 & - & - \\
\hline \multicolumn{6}{|c|}{ DGRC0025 - t(12;17) } \\
\hline inv Fully Rslv & Cluster (7) & Clu[GRCh38]3p21.31(50,130,322-50,135,115)inv & 4.80 & - & SEMA3F-AS1 \\
\hline inv Fully Rslv & Cluster (3) & Clu[GRCh38]3p12.3(79,609,499-79,616,630)inv & 7.13 & $R O B O 1$ & - \\
\hline inv Fully Rslv & Cluster (5) & Clu[GRCh38]3q25.32(159,075,698-159,081,455)inv & 5.76 & IQCJ & - \\
\hline inv Fully Rslv & Cluster (5) & Clu[GRCh38]7q31.32(121,520,333-121,526,336)inv & 6.00 & - & - \\
\hline inv Fully Rslv & Cluster (4) & Clu[GRCh38]10q21.3(65,664,415-65,671,542)inv & 7.13 & - & LINC01515 \\
\hline inv Fully Rslv & Cluster (5) & Clu[GRCh38]15q26.2(97,353,880-97,359,017)inv & 5.14 & - & LINC02253 \\
\hline $\begin{array}{l}\text { interchr ins } \\
\text { Fully Rslv }\end{array}$ & Cluster (7) & $\begin{array}{l}\text { Excision: Clu[GRCh38]3p11.1(89,459,946-89,460,378) } \\
\text { Insertion: Clu[GRCh38]1p12(119,252,479-119,255,488) }\end{array}$ & 0.432 & EPHA3 & $R P 11-418 J 17$ \\
\hline $\begin{array}{l}\text { interchr ins } \\
\text { Fully Rslv }\end{array}$ & Cluster (8) & $\begin{array}{l}\text { Excision: Clu[GRCh38]10p12.33(17,548,358-17,548,457) } \\
\text { Insertion: Clu[GRCh38]3p21.31(49,401,798-49,406,995) }\end{array}$ & 0.099 & $\overline{R H O A}$ & - \\
\hline
\end{tabular}




\begin{tabular}{|c|c|c|c|c|}
\hline & DGRC0030 - t(1;3) & & & \\
\hline inv Fully Rslv & Cluster (3) Clu[GRCh38]5q32(146,449,266-146,454,922)inv & 5.66 & TCERG1 & - \\
\hline inv Fully Rslv & Clu[GRCh38]7p12.1(52,318,497-52,324,132)inv & 5.64 & - & - \\
\hline $\begin{array}{l}\text { dellNVdel } \\
\text { Fully Rslv }\end{array}$ & \begin{tabular}{ll}
\multirow{3}{*}{ Cluster (4) } & Clu[GRCh38]11p15.5(1,351,552-1,354,130)del \\
& Clu[GRCh38]11p15.5(1,354,130-1,354,199)inv \\
& Clu[GRCh38]11p15.5(1,354,199-1,356,719)del
\end{tabular} & 5.17 & - & AC136297.1 \\
\hline
\end{tabular}

Analysis performed with the reported workflow and CNV-ConTool with minimum reciprocal overlap of $70 \%$. Individual SVs within a cx variant are designated in lowercase and uppercase letters. ${ }^{a}$ Values in parentheses indicate number of read-pairs per sequence cluster. ${ }^{b} \mathrm{Clu}$, sequence cluster. ${ }^{\circ}$ Genes with OMIM number, without or without an associated phenotype. IInversion breakpoint is validated by a split-read. Rslv, Resolved. 
Supplementary Table 16 Expression of genes within disrupted hESC TADs in LCLs with DGRC0016 t(16;17)

\begin{tabular}{|c|c|c|c|c|c|c|c|c|c|}
\hline \multirow{2}{*}{$\begin{array}{l}\text { Transcript } \\
\text { Cluster ID }\end{array}$} & \multirow[b]{2}{*}{ Gene Symbol } & \multirow[b]{2}{*}{ Description } & \multirow[b]{2}{*}{ Group } & \multirow[b]{2}{*}{ Strand } & \multirow[b]{2}{*}{ Chr. } & \multirow[b]{2}{*}{ Genomic Position } & \multicolumn{2}{|c|}{$\begin{array}{l}\text { Bi-weight Avg } \\
\text { Signal }(\log 2) \\
\end{array}$} & \multirow{2}{*}{$\begin{array}{l}\text { LCLs } \\
\text { SD }\end{array}$} \\
\hline & & & & & & & $\begin{array}{l}\text { Proband's } \\
\text { LCL }\end{array}$ & $\begin{array}{l}\text { LCLs } \\
(n=4)\end{array}$ & \\
\hline \multicolumn{10}{|c|}{ The $16 q 24.3$ breakpoint within hESC TAD at chr16:89246091-89686091 } \\
\hline TC16001350.hg.1 & ANKRD11 & Ankyrin repeat domain 11 & Coding & - & $16 q 24.3$ & $89,267,619-89,490,561$ & 7.77 & 7.85 & 0.09 \\
\hline TC16000695.hg.1 & LOC100287036 & Uncharacterized LOC100287036 & Coding & + & $16 q 24.3$ & $89,321,133-89,325,110$ & 4.03 & 4.03 & 0,05 \\
\hline TC16001711.hg.1 & LOC101927817 & Uncharacterized LOC101927817 & NonCoding & + & $16 q 24.3$ & $89,430,918-89,454,494$ & 5.15 & 5.23 & 0.15 \\
\hline TC16000696.hg.1 & LOC101927817 & Uncharacterized LOC101927817 & Coding & + & $16 q 24.3$ & $89,430,918-89,454,494$ & 4.81 & 4.85 & 0,12 \\
\hline TC16000698.hg.1 & $\begin{array}{l}\text { SPG7; } \\
\text { LOC101930112 }\end{array}$ & $\begin{array}{l}\text { Spastic paraplegia } 7 \text { (pure and complicated autosomal } \\
\text { recessive); uncharacterized LOC101930112 }\end{array}$ & Coding & + & $16 q 24.3$ & $89,490,719-89,557,768$ & 7.13 & 7.12 & 0 \\
\hline TC16001713.hg.1 & $R P L 13$ & Ribosomal protein L13 & NonCoding & + & $16 q 24.3$ & $89,560,657-89,566,828$ & 14.27 & 15.06 & 0.77 \\
\hline TC16000699.hg.1 & SNORD68 & Ribosomal protein L13; small nucleolar RNA, C/D box 68 & Coding & + & $16 q 24.3$ & $89,561,434-89561517$ & 7.13 & 7.11 & 0.13 \\
\hline TC16000700.hg.1 & CPNE7 & Copine VII & Coding & + & $16 q 24.3$ & $89,575,768-89,597,246$ & 6.86 & 6.92 & 0.380 \\
\hline TC16000701.hg.1 & DPEP1 & Dipeptidase 1 (renal) & Coding & + & $16 q 24.3$ & $89,613,308-89,638,456$ & 5.4 & 5.4 & 0.02 \\
\hline TC16001353.hg.1 & CHMP1A & Charged multivesicular body protein $1 \mathrm{~A}$ & Coding & - & $16 q 24.3$ & $89,644,431-89,657,845$ & 7.78 & 7.73 & 0.24 \\
\hline TC16000702.hg.1 & SPATAЗ3 & $\begin{array}{l}\text { Spermatogenesis associated 33; chromosome } 16 \text { open } \\
\text { reading frame } 55\end{array}$ & Coding & + & $16 q 24.3$ & $89,657,802-89,671,272$ & 5.17 & 5.17 & 0 \\
\hline TC16000703.hg.1 & $C D K 10$ & Cyclin-dependent kinase 10 & Coding & + & $16 q 24.3$ & $89,680,737-89,696,364$ & 6.86 & 6.9 & 0.24 \\
\hline \multicolumn{10}{|c|}{ 17q21.31 breakpoint within hESC TAD at chr17:46687454-47647635 } \\
\hline TC17000600.hg.1 & $\begin{array}{l}\text { NSF; NSFP1; } \\
\text { LOC101930324 }\end{array}$ & $\begin{array}{l}\text { N-ethylmaleimide-sensitive factor; N-ethylmaleimide- } \\
\text { sensitive factor pseudogene } 1\end{array}$ & Coding & + & $17 q 21.31$ & $46,590,669-46,757,464$ & 9.53 & 9.56 & 0.27 \\
\hline TC17001615.hg.1 & $\begin{array}{l}\text { WNT3; } \\
\text { LOC101929777 }\end{array}$ & $\begin{array}{l}\text { Wingless-type MMTV integration site family, member } \\
\text { 3; uncharacterized LOC101929777 }\end{array}$ & Coding & - & $17 q 21.31$ & $46,762,506-46,833,154$ & 5.86 & 4.6 & 0.01 \\
\hline TC17000601.hg.1 & WNT9B & Wingless-type MMTV integration site family, member 9B & Coding & + & $17 q 21.32$ & $46,833,201-46,886,730$ & 4.69 & 4.69 & 0.05 \\
\hline TC17000603.hg.1 & GOSR2 & Golgi SNAP receptor complex member 2 & Coding & + & $17 q 21.32$ & $46,923,075-46,975,524$ & 9.26 & 9.25 & 0.12 \\
\hline TC17001616.hg.1 & $R P R M L$ & Reprimo-like & Coding & - & $17 q 21.32$ & $46,978,156-46,979,248$ & 4.83 & 4.81 & 0.14 \\
\hline TC17001621.hg.1 & $C D C 27$ & Cell division cycle 27 & Coding & - & $17 q 21.32$ & $47,117,703-47,189,422$ & 11.78 & 11.72 & 0.24 \\
\hline TC17000606.hg.1 & MYL4 & Myosin, light chain 4, alkali; atrial, embryonic & Coding & + & $17 q 21.32$ & $47,200,446-47,223,679$ & 4.57 & 4.57 & 0.06 \\
\hline TC17002878.hg.1 & ITGB3 & Integrin, beta 3 (platelet glycoprotein IIla, antigen CD61) & Coding & + & $17 q 21.32$ & $47,253,846-47,311,816$ & 7.45 & 7.59 & 1.5 \\
\hline TC17002879.hg.1 & $\begin{array}{l}\text { EFCAB13; } \\
\text { C17orf57 }\end{array}$ & $\begin{array}{l}\text { EF-hand calcium binding domain 13; chromosome } 17 \\
\text { open reading frame } 57\end{array}$ & Coding & + & $17 q 21.32$ & $47,323,290-47,441,312$ & 6.51 & 6.37 & 0.37 \\
\hline TC17001625.hg.1 & MRPL45P2 & Mitochondrial ribosomal protein L45 pseudogene & Coding & - & $17 q 21.32$ & $47,450,568-47,492,492$ & 8.27 & 8.34 & 0.2 \\
\hline TC17000609.hg.1 & $\begin{array}{l}\text { NPEPPS; } \\
\text { LOC100653042 }\end{array}$ & $\begin{array}{l}\text { Aminopeptidase puromycin sensitive; uncharacterized } \\
\text { LOC100653042 }\end{array}$ & Coding & + & $17 q 21.32$ & $47,522,942-47,623,276$ & 11.49 & 11.49 & 0.12 \\
\hline
\end{tabular}

Genes disrupted by breakpoints are in bold. A statistically significant linear fold change of 2.6 of the disrupted WNT3 was identified. 
Supplementary Table 17 Protein coding and non-coding RNA genes localized within hESC TADs disrupted by DGRC0006 $\mathrm{t}(8 ; 14)$ breakpoints, their associated phenotypes and GWAS data from the referred regions

\begin{tabular}{|c|c|c|c|c|}
\hline \multicolumn{3}{|c|}{ Genes and intergenic regions } & \multirow{2}{*}{$\begin{array}{c}\text { Clinical Phenotype } \\
\text { Phenotype;\#OMIM_Inheritance; } \\
\text { DDG2P_category } \\
\end{array}$} & \multirow{2}{*}{$\begin{array}{c}\text { GWAS SNP data } \\
\text { Genetic traits }\end{array}$} \\
\hline GeneCard & OMIM & oe $(\mathrm{Cl} 95 \%)$ & & \\
\hline \multicolumn{5}{|c|}{ 8q12.3 breakpoint within hESC TAD at 8:63204888-64444889 } \\
\hline YTHDF3 & na & $0.05(0.02-0.23)$ & None & None \\
\hline AC011978 & na & nd & None & None \\
\hline AC011124 & na & nd & None & None \\
\hline AC018953 & na & nd & None & None \\
\hline \multicolumn{3}{|c|}{ Intergenic - chr8:63589408-63687179 } & None & \# 1 SNPs - EFO:0004342_Waist circumference [6.92E-6]; DOID:9970_Obesity [6.92E-6] \\
\hline AC069133 & na & nd & None & None \\
\hline LINC01289 & na & nd & None & \# 2 SNPs - EFO:0004342_Waist circumference [6.92E-6]; DOID:9970_Obesity [6.92E-6] \\
\hline RP11-32K4.1-IVS1 & na & nd & None & \# 2 SNPs - EFO:0004342_Waist circumference [6.92E-6]; DOID:9970_Obesity [6.92E-6] \\
\hline \multicolumn{5}{|c|}{ der(8)g.[chr8:pter_cen_64209134::chr14:83126596_83126598dup_qter] } \\
\hline \multicolumn{3}{|c|}{ Intergenic - chr8:64209111-64373328 } & None & \# 49 SNPs - EFO:0004342_Waist circumference [6.92E-6]; DOID:9970_Obesity [6.92E-6] \\
\hline MIR124-2HG & na & nd & None & \# 1 SNPs - EFO:0004342_Waist circumference [6.92E-6]; DOID:9970_Obesity [6.92E-6] \\
\hline \multicolumn{5}{|c|}{ 14q31.2 breakpoint within hESC interTAD region at chr14:83103903-83503903 } \\
\hline \multicolumn{5}{|c|}{ der(14) g.[chr14:pter_cen_83126598:::chr8:64209135_qter] $\quad$ None } \\
\hline \multicolumn{5}{|c|}{$\begin{array}{l}\text { Table prepared with TAD-GConTool that includes GWAS SNP data FANTOM CAT database (Hon et al. 2017). SNP associated genetic } \\
\text { traits are designated according to their ontology term ID. All p-values are below GWAS significance threshold of } \leq 5.00 E-8 \text {. Clinica } \\
\text { phenotype data are from OMIM and DDG2P databases. The clinical phenotype data are configured as follows: i) gene-associatec } \\
\text { phenotypes in humans from either database; ii) \#OMIM phenotype number underscore type of inheritance (AR, AD or AR/AD) or \#NA ir } \\
\text { the absence of OMIM phenotype number; and DDG2P underscore phenotype classification (confirmed, probable and possible) ol } \\
\text { DDG2P NA in the absence of DDG2P data. }\end{array}$} \\
\hline
\end{tabular}


Supplementary Table 18 Protein coding and non-coding RNA genes localized within hESC TADs disrupted by DGRC0013 inv(13) breakpoints with their associated phenotypes and GWAS data from the referred regions
Genes and intergenic regions
Clinical Phenotype
GWAS SNP data
GeneCard OMIM oe (CI 95\%)
Phenotype;\#OMIM_Inheritance;
Genetic traits

13q12.3 breakpoint within hESC TAD at 13:28007863-28807863

FLT3 $\quad 136351 \quad 0.22(0.14-0.35)$

Leukemia, acute lymphoblastic, somatic

\#613065_nd; DDG2P_NA

Leukemia, acute myeloid, somatic

\#601626_nd; DDG2P_NA

None

\begin{tabular}{ll}
\hline Intergenic - chr13:28100592-28138506 \\
\hline PAN3 $617448 \quad 0.05(0.02-0.15)$
\end{tabular}

\begin{tabular}{lll} 
PAN3 & 617448 & $0.05(0.02-0.15)$ \\
\hline
\end{tabular}

None

FLT1 - IVS1 $165070 \quad 0.14(0.09-0.24)$

None

[9.64E-13]; DOID:1574_Alcohol abuse [9.64E-13]; DOID:0050741_Alcohol dependence [9.64E-13]

None

\# 28 SNPs - MESH:D003327 Coronary disease [6E-07]

\# 24 SNPs - EFO:0004731_Eye measurement [9.75E-7]; DOID:2462_Retinal vascular disease [9.75E-7]

\# 3 SNPs - HP:0000707_Abnormality of the nervous system [9.64E-13]; EFO:0004329_Alcohol drinking

[9.64E-13]; DOID:1574_Alcohol abuse [9.64E-13]; DOID:0050741_Alcohol dependence [9.64E-13]

\# 1 SNPs - MESH:D008076 Cholesterol hdl [1.24E-6]; EFO:0004612 High density lipoprotein cholesterol measurement [1.24E-6]; DOID:1461_Cholesterol embolism [1.24E-6]

g.[pter_cen_28489792_74831812inv;74831813_74831818del_qter]

Intergenic - chr13:28489792-28659104

\# 18 SNPs - HP:0003002 Breast carcinoma [7.90E-6]

\# 2 SNPs - DOID:1574 Alcohol abuse [9.64E-13]; DOID:0050741 Alcohol dependence [9.64E-13]

HP:0000707_Abnormality of the nervous system [9.64E-13]; EFO:0004329_Alcohol drinking [9.64E-13]

\# 1 SNPs - DOID:12603_Acute leukemia [6E-6]; DOID:9952_Acute lymphocytic leukemia [6E-6]

\begin{tabular}{lccc}
\hline POMP & $613386 \quad 0.00(0.00-0.39)$ & $\begin{array}{c}\text { Keratosis linearis with ichthyosis congenita } \\
\text { and sclerosing keratoderma; \#601952_AR; } \\
\text { DDG2P_possible }\end{array}$ & None \\
& $\begin{array}{c}\text { Proteasome-associated autoinflammatory } \\
\text { syndrome 2; \#618048_AD; DDG2P_NA }\end{array}$ & \\
\hline Intergenic - chr13:28678925-28700064 & None & None \\
\hline SLC46A3 $\quad 616764 \quad 0.75$ & None & None \\
\hline Intergenic - chr13:28718970-28807863 & None & None
\end{tabular}

\section{3q22.1 breakpoint within hESC TAD at 13:73727862-75327863}

$\begin{array}{rrr}13 q 212 & \text { None }\end{array}$

\# 9 SNPs - HP:0005344_Abnormality of the carotid arteries [7.90E-6]

\# 6 SNPs - HP:0001699_Sudden death [1E-8]; DOID:10273_Heart conduction disease [1E-8]

\# 1 SNPs - HP:0001645 Sudden cardiac death [5E-20]

Intergenic - chr13:73995056-74231457 None \# 49 SNPs - HP:0001645_Sudden cardiac death [5E-20]

LINC00402 na nd None

\# 1 SNPs - DOID:8986_Narcolepsy [3.59E-25]; HP:0100786_Hypersomnia [3.59E-25]

None 


\begin{tabular}{|c|c|c|}
\hline Intergenic - chr13:74259976-74412957 & None & None \\
\hline AL355390 na $\quad$ nd & None & None \\
\hline LINC00381 na & None & None \\
\hline Intergenic - chr13:74435159-74552503 & None & $\begin{array}{l}\text { \# 68 SNPs - HP:0005368_Abnormality of humoral immunity [3E-7]; DOID:8736_Smallpox [3E-7]; } \\
\text { EFO:0004873_Response to vaccine [3E-7] } \\
\text { \# 11 SNPs - DOID:9352_Type 2 diabetes mellitus [1E-8]; EFO:0004639_Phospholipid measurement [1E-08] }\end{array}$ \\
\hline LINC00347 na $\quad$ nd & None & \# 10 SNPs - EFO:0004639_Phospholipid measurement [1E-08]; DOID:9352_Type 2 diabetes mellitus [1E-8] \\
\hline Intergenic - chr13:74565445-74831722 & None & \# 2 SNPs - EFO:0004639_Phospholipid measurement [1E-8]; DOID:9352_Type 2 diabetes mellitus [1E-8] \\
\hline \multicolumn{3}{|c|}{ g.[pter_cen_28489792_74831812inv;74831813_74831818del_qter] } \\
\hline Intergenic - chr13:74831722-75250480 & None & \# 6 SNPs - DOID:6000_Congestive heart failure [6E-7]; MESH:D006333_Heart failure [6E-7] \\
\hline LINC01078 na nd & None & None \\
\hline Intergenic - chr13:75252012-75284665 & None & None \\
\hline 6124650.71 & $\begin{array}{l}\text { Diabetes mellitus, noninsulin-dependent, 5; } \\
\quad \text { \#616087_nd; DDG2P_NA }\end{array}$ & $\begin{array}{l}\text { None } \\
\text { \# } 1 \text { SNPs - DOID:9744 Tvpe } 1 \text { diabetes mellitus [6E-11] }\end{array}$ \\
\hline
\end{tabular}

Table prepared with TAD-GConTool that includes GWAS SNP data from FANTOM CAT database (Hon et al. 2017). SNP associated genetic traits are presented according to their ontology term ID. Although conventional GWAS significance threshold is $\leq 5.00 \mathrm{E}-8$, for disrupted genes, adjacent genomic regions and for genetic traits identified in GWAS (in bold), overlapping the proband's clinical phenotype, a threshold of 9.99E6 is used. Clinical phenotype data are from OMIM and DDG2P databases. The clinical phenotype data are configured as follows i) geneassociated phenotypes in humans from either database; ii) \#OMIM phenotype number underscore type of inheritance (AR, AD or AR/AD) or \#NA in the absence of OMIM phenotype number; and DDG2P underscore phenotype classification (confirmed, probable and possible) or DDG2P_NA in the absence of DDG2P data. 
Supplementary Table 19 Protein coding and non-coding RNA genes localized within hESC TADs disrupted by DGRC0025 t(12;17) breakpoints with their associated phenotypes and GWAS data from the referred regions

\begin{tabular}{|c|c|c|c|c|}
\hline \multicolumn{3}{|c|}{ Genes and intergenic regions } & \multirow{2}{*}{$\begin{array}{c}\text { Clinical Phenotype } \\
\text { Phenotype;\#OMIM_Inheritance; } \\
\text { DDG2P_category }\end{array}$} & \multirow{2}{*}{$\begin{array}{c}\text { GWAS SNP data } \\
\text { Genetic traits }\end{array}$} \\
\hline GeneCard & OMIM & oe $(\mathrm{Cl} 95 \%)$ & & \\
\hline \multicolumn{5}{|c|}{ 12q23.1 breakpoint within hESC TAD at 12:99042091-99802091 } \\
\hline $\operatorname{der}(12) \mathrm{g} .[\mathrm{chr} 12$ & 607815 & $0.10(0.05-0.20)$ & :::chr17:51565697_qter] & $\begin{array}{l}\text { \# } 119 \text { SNPs - DOID:5419_Schizophrenia [4E-7] } \\
\text { \# } 90 \text { SNPs - DOID:1094_Attention deficit hyperactivity disorder [2E-6]; HP:0007302_Bipolar affective } \\
\text { disorder [2E-6]; HP:0000729_Autistic behavior [2E-6]; DOID:1470_Major depressive disorder [2E-6] } \\
\text { \# } 33 \text { SNPs - DOID:9970_Obesity [7E-6] } \\
\text { \# } 6 \text { SNPs - DOID:332_Amyotrophic lateral sclerosis [8E-6] }\end{array}$ \\
\hline FAM71C & na & $0.31(0.13-0.99)$ & None & None \\
\hline Intergenic - chr12 & $650046-9$ & 802091 & None & None \\
\hline \multicolumn{5}{|c|}{ 17q21.33 breakpoint within hESC TAD at 17:51287640-54967640 } \\
\hline UTP18 & 612816 & 0.37 & None & None \\
\hline LINC02071 & na & nd & None & None \\
\hline AC005823 & na & nd & None & None \\
\hline LINC02072 & na & nd & None & None \\
\hline LINC02073 & & nd & None & None \\
\hline Intergenic - chr17 & $445802-5$ & 565697 & None & $\begin{array}{l}\text { \# } 4 \text { SNPs - DOID:9352_Type } 2 \text { diabetes mellitus [7E-13]; HP:0000140_Abnormality of the menstrual cycle } \\
\text { [7E-13]; MESH:D011293_Premenstrual syndrome [7E-13]; DOID:10652_Alzheimer s disease [7E-13]; } \\
\text { DOID:11476_Osteoporosis [7E-13]; DOID:9970_Obesity [7E-13]; DOID:1612_Breast cancer [7E-13] }\end{array}$ \\
\hline \multicolumn{5}{|c|}{ der(17) g.[chr17:pter_cen_51565696::chr12:99637782_qter] } \\
\hline CA10 & 604642 & $0.21(0.10-0.47)$ & None & None \\
\hline \multicolumn{3}{|c|}{ Intergenic - chr17:52160017-52390515 } & None & None \\
\hline LINC01982 & na & nd & None & None \\
\hline LINC02089 & na & nd & None & None \\
\hline C17orf112 & na & $0.28(0.10-1.30)$ & None & None \\
\hline \multicolumn{3}{|c|}{ Intergenic - chr17:52987652-53822901 } & None & None \\
\hline KIF2B & 615142 & 0.89 & None & None \\
\hline \multicolumn{3}{|c|}{ Intergenic - chr17:53825213-54899387 } & None & $\begin{array}{l}\text { \# } 24 \text { SNPs - DOID:1574_Alcohol abuse [7.87E-20]; DOID:0050741_Alcohol dependence [7.87E-20]; } \\
\text { HP:0000707_Abnormality of the nervous system [7.87E-20]; EFO:0004329_Alcohol drinking [7.87E-20] }\end{array}$ \\
\hline TOM1L1 & 604701 & 0.68 & None & \# 25 SNPs - HP:0003002_Breast carcinoma [2E-13] \\
\hline COX11 & 603648 & 0.40 & None & \# 11 SNPs - HP:0003002_Breast carcinoma [2E-13] \\
\hline
\end{tabular}


Table prepared with TAD-GConTool that includes GWAS SNP data FANTOM CAT database (Hon et al. 2017). SNP associated genetic traits are presented according to their ontology term ID. Although conventional GWAS significance threshold is $\leq 5.00 \mathrm{E}-8$, for disrupted genes and adjacent genomic regions a threshold of 9.99E-6 is used. Clinical phenotype data are from OMIM and DDG2P databases. 
Supplementary Table S20 Protein coding and non-coding RNA genes localized within hESC TADs disrupted by DGRC0030 t(1;3) breakpoints with their associated phenotypes and GWAS data from the referred regions

\section{GWAS SNP data}

\begin{tabular}{ccc}
\hline Genes and intergenic regions & & Clinical Phenotype \\
GeneCard OMIM $\quad$ oe (Cl $95 \%)$ & & $\begin{array}{c}\text { Phenotype; \#OMIM_Inheritance; } \\
\text { DDG2P category }\end{array}$
\end{tabular}

\section{1q42.11 breakpoint within hESC TAD at 1:224025675-224425675}

Intergenic - chr1:224025675-22411408

\begin{tabular}{llll}
\hline FBXO28 609100 & $0.06(0.02-0.29)$ & None
\end{tabular}

Intergenic - chr1:224162047-224175756

None

GWAS SNP data
Genetic traits

\# 10 SNPs - DOID:614_Lymphopenia [3.66E-8]; DOID:2841_Asthma [3.66E-8]; HP:0002665_Lymphoma [3.66E8]; DOID:1240_Leukemia [3.66E-8]

\# 43 SNPs - DOID:1240_Leukemia [3.66E-8]; HP:0002665_Lymphoma [3.66E-8]; DOID:614_Lymphopenia [3.66E-8]; DOID:2841_Asthma [3.66E-8]

\begin{tabular}{|c|c|c|c|}
\hline \multicolumn{2}{|c|}{ Intergenic - chr'1:22416204/-2241/5/56 } & None & DOID:614_Lymphopenia [3.66E-8] \\
\hline DEGS1 & $6158430.30(0.14-0.77)$ & $\begin{array}{l}\text { Leukodystrophy, hypomyelinating, } \\
\text { 18; \#618404_AR;DDG2P_NA }\end{array}$ & $\begin{array}{l}\text { \# 1 SNPs - DOID:1240_Leukemia [3.66E-8]; HP:0002665_Lymphoma [3.66E-8]; DOID:614_Lymphopenia [3.66E- } \\
\text { 8]; DOID:2841_Asthma [3.66E-8] }\end{array}$ \\
\hline$N V L$ & $602426 \quad 0.70$ & None & \# 74 SNPs - DOID:9255_Frontotemporal dementia [5.30E-26]; MESH:D009203_Myocardial infarction [5.30E-26] \\
\hline Intergenic - & chr1:224330387-224356850 & None & \# 33 SNPs - DOID:9255_Frontotemporal dementia [5.30E-26]; MESH:D009203_Myocardial infarction [5.30E-26] \\
\hline $\mathrm{CNIH4}$ & $617483 \quad 0.43$ & None & None \\
\hline $\begin{array}{l}\text { WDR26 - } \\
\text { IVS5 }\end{array}$ & $6174240.00(0.00-0.08)$ & $\begin{array}{l}\text { Skraban-Deardorff syndrome; } \\
\text { \#617616_AD;DDG2P_confirmed }\end{array}$ & $\begin{array}{l}\text { \# 1 SNPs - DOID:3083_Chronic obstructive pulmonary disease [4.05E-6]; MESH:D008171_Lung diseases } \\
\text { [4.05E-6]; DOID:2841_Asthma [4.05E-6]; HP:0002795_Functional respiratory abnormality [4.05E-6]; } \\
\text { EFO:0004713 Forced expiratory volume [4.05E-6] }\end{array}$ \\
\hline
\end{tabular}

der(1) g.[chr1:pter_cen_224398161_224398171del::chr3:pter_10670893inv]

3p25.3 breakpoint within hESC TAD at 3:10343316-10743315

ATP2B2 - $1087330.06(0.03-0.15) \quad$ Deafness, autosomal recessive 12,
IVS1

modifier of

\#601386_AR;DDG2P NA

\# 73 SNPs - HP:0100786 Hypersomnia [2.51E-9]; DOID:8986 Narcolepsy [2.51E-9]

\# 8 SNPs - MESH:D015992_Body mass index [2.82E-6]; HP:0001507_Growth abnormality [2.82E-6]; DOID:9970_Obesity [2.82E-6]]

\# 7 SNPs - DOID:332 Amyotrophic lateral sclerosis [7E-6]

\# 2 SNPs - DOID:9975_Cocaine dependence [3.30E-6]; HP:0000707_Abnormality of the nervous system [3.30E-6]

der(3) g.[chr1:pter_224398172inv::chr3: 10670894_10670895del_cen_qter]

Intergenic - chr3:10670895-10743315

None

\# 12 SNPs - DOID:1561 Cognitive disorder [1.02E-6]

Table prepared with TAD-GConTool that includes GWAS SNP data from FANTOM CAT database (Hon et al. 2017). Genes showing high probability of being AD disease causing are in bold (McKusick 1998; Wright et al. 2015). SNP associated genetic traits are presented according to their ontology term ID. Although conventional GWAS significance threshold is $\leq 5.00 \mathrm{E}-8$, for disrupted genes and adjacent genomic regions a threshold of 9.99E-6 is used. Clinical phenotype data are from OMIM and DDG2P databases. The clinical phenotype data are configured as follows: i) gene-associated phenotypes in humans from either database; ii) \#OMIM phenotype number underscore type of inheritance (AR, AD or AR/AD) or \#NA in the absence of OMIM phenotype number; and DDG2P underscore phenotype classification (confirmed, probable and possible) or DDG2P_NA in the absence of DDG2P data. 


\section{Supplementary References}

Auton A, Abecasis G, Altshuler D, et al. (2015) A global reference for human genetic variation. Nature 526:68-74.

Bult CJ, Eppig JT, Kadin JA, Richardson JE, Blake JA, Mouse Genome Database Group. (2008) The Mouse Genome Database (MGD): mouse biology and model systems. Nucleic Acids Res 36:D724-728.

Carbonell AU, Cho CH, Tindi JO, Counts PA, Bates JC, Erdjument-Bromage H, Cvejic S, laboni A, Kvint I, Rosensaft J, Banne E, Anagnostou E, Neubert TA, Scherer SW, Molholm S, Jordan BA. (2019) Haploinsufficiency in the ANKS1B gene encoding AIDA-1 leads to a neurodevelopmental syndrome. Nat Commun 10:3529.

Cock PJA, Antao T, Chang JT, Chapman BA, Cox CJ, Dalke A, Friedberg I, Hamelryck T, Kauff F, Wilczynski B, de Hoon MJL. (2009) Biopython: freely available Python tools for computational molecular biology and bioinformatics. Bioinformatics 25:1422-1423.

Coe BP, Witherspoon K, Rosenfeld JA, van Bon BWM, Vulto-van Silfhout ATV, Bosco $\mathrm{P}$, Friend $\mathrm{KL}$, Baker $\mathrm{C}$, Buono S, Vissers LELM, Schuurs-Hoeijmakers $\mathrm{JH}$, Hoischen A, Pfundt R, Krumm N, Carvill GL, Li D, Amaral D, Brown N, Lockhart PJ, Scheffer IE, Alberti A, Shaw M, Pettinato R, Tervo R, de Leeuw N, Reijnders MRF, Torchia BS, Peeters H,Thompson E, O'Roak BJ, Fichera M, Hehir-Kwa JY, Shendure J, Mefford HC, Haan E, Gécz J, de Vries BBA, Romano C, Eichler EE. (2014) Refining analyses of copy number variation identifies specific genes associated with developmental delay. Nat Genet 46:1063-1071.

Collins RL, Stone MR, Brand H, Glessner JT, Talkowski ME. (2016) CNView: a visualization and annotation tool for copy number variation from whole-genome sequencing. bioRxiv 049536; (CNView, https://github.com/RCollins13/CNView).

Collins RL, Brand H, Redin CE, Hanscom C, Antolik C, Stone MR, Glessner JT, Mason T, Pregno G, Dorrani N, Mandrile G, Giachino D, Perrin D, Walsh C, Cipicchio M, Costello M, Stortchevoi A, An JY, Currall BB, Seabra CM, Ragavendran A, Margolin L, Martinez-Agosto JA, Lucente D, Levy B, Sanders SJ, Wapner RJ, Quintero-Rivera F, Kloosterman W, Talkowski ME. (2017) Defining the diverse spectrum of inversions, complex structural variation, and chromothripsis in the morbid human genome. Genome Biol 18:36.

Cooper GM, Coe BP, Girirajan S, Rosenfeld JA, Vu TH, Baker C, Williams C, Stalker H, Hamid R, Hannig V, Abdel-Hamid H, Bader P, McCracken E, Niyazov D, Leppig K, Thiese H, Hummel M, Alexander N, Gorski J, Kussmann J, Shashi V, Johnson K, Rehder C, Ballif BC, Shaffer LG, Eichler EE. (2011) A copy number variation morbidity map of developmental delay. Nat Genet 43:838-846.

Dixon JR, Selvaraj S, Yue F, Kim A, Li Y, Shen Y, Hu M, Liu JS, Ren B. (2012) Topological domains in mammalian genomes identified by analysis of chromatin interactions. Nature 485:376-380.

Durand NC, Robinson JT, Shamim, MS, Machol I, Mesirov JP, Lander ES, Aiden EL. (2016) Juicebox provides a visualization system for $\mathrm{Hi}-\mathrm{C}$ contact maps with unlimited zoom. Cell Systems 3:99-101.

Firth HV, Richards SM, Bevan AP, Clayton S, Corpas M, Rajan D, Vooren SV, Moreau Y, Pettett RM, Carter NP. (2009) DECIPHER: database of chromosomal 
imbalance and phenotype in humans using Ensembl resources. Am J Hum Genet 84:524-533.

Hodgetts Morton V, Quinlan-Jones E, Butts N, Williams D, Hamilton S, Marton T, Morris K. (2017) The first antenatal diagnosis of KBG syndrome: a microdeletion at chromosome 16q24.2q24.3 containing multiple genes including ANKRD11 associated with the disorder. Clin Case Rep 6:189-191.

Hon CC, Ramilowski JA, Harshbarger J, Bertin N, Rackham OJ, Gough J, Denisenko E, Schmeier S, Poulsen TM, Severin J, Lizio M, Kawaji H, Kasukawa T, Itoh M, Burroughs AM, Noma S, Djebali S, Alam T, Medvedeva YA, Testa AC, Lipovich L, Yip CW, Abugessaisa I, Mendez M, Hasegawa A, Tang D, Lassmann T, Heutink P, Babina M, Wells CA, Kojima S, Nakamura Y, Suzuki H, Daub CO, de Hoon MJ, Arner E, Hayashizaki Y, Carninci P, Forrest AR. (2017) An atlas of human long non-coding RNAs with accurate 5' ends. Nature 543:199-204.

Huang N, Lee I, Marcotte EM, Hurles ME. (2010) Characterising and predicting haploinsufficiency in the human genome. PLoS Genet 6:e1001154.

Kaminsky EB, Kaul V, Paschall J, Church DM, Bunke B, Kunig D, Moreno-De-Luca D, Moreno-De-Luca A, Mulle JG, Warren ST, Richard G, Compton JG, Fuller AE, Gliem TJ, Huang S, Collinson MN, Beal SJ, Ackley T, Pickering DL, Golden DM, Aston E, Whitby $H$, Shetty S, Rossi MR, Rudd MK, South ST, Brothman AR, Sanger WG, lyer RK, Crolla JA, Thorland EC, Aradhya S, Ledbetter DH, Martin CL. (2011) An evidence-based approach to establish the functional and clinical significance of copy number variants in intellectual and developmental disabilities. Genet Med 13:777-784.

Landrum MJ, Lee JM, Benson M, Brown G, Chao C, Chitipiralla S, Gu B, Hart J, Hoffman D, Hoover J, Jang W, Katz K, Ovetsky M, Riley G, Sethi A, Tully R, Villamarin-Salomon R, Rubinstein W, Maglott DR. (2016) ClinVar: public archive of interpretations of clinically relevant variants. Nucleic Acids Res 44:D862-868.

Layer RM, Chiang C, Quinlan AR, Hall IM. (2014) LUMPY: a probabilistic framework for structural variant discovery. Genome Biol 15:R84.

Lek M, Karczewski KJ, Minikel EV, Samocha KE, Banks E, Fennell T, O'Donnell-Luria AH, Ware JS, Hill AJ, Cummings BB, Tukiainen T, Birnbaum DP, Kosmicki JA, Duncan LE, Estrada K, Zhao F, Zou J, Pierce-Hoffman E, Berghout J, Cooper DN, Deflaux N, DePristo M, Do R, Flannick J, Fromer M, Gauthier L, Goldstein J, Gupta N, Howrigan D, Kiezun A, Kurki MI, Moonshine AL, Natarajan P, Orozco L, Peloso GM, Poplin R, Rivas MA, Ruano-Rubio V, Rose SA, Ruderfer DM, Shakir K, Stenson PD, Stevens C, Thomas BP, Tiao G, Tusie-Luna MT, Weisburd B, Won $\mathrm{HH}, \mathrm{Yu}$ D, Altshuler DM, Ardissino D, Boehnke M, Danesh J, Donnelly S, Elosua R, Florez JC, Gabriel SB, Getz G, Glatt SJ, Hultman CM, Kathiresan S, Laakso M, McCarroll S, McCarthy MI, McGovern D, McPherson R, Neale BM, Palotie A, Purcell SM, Saleheen D, Scharf JM, Sklar P, Sullivan PF, Tuomilehto J, Tsuang MT, Watkins HC, Wilson JG, Daly MJ, MacArthur DG, Exome Aggregation Consortium. (2016) Analysis of protein-coding genetic variation in 60,706 humans. Nature 536:285-291.

Li H, Durbin R. (2009) Fast and accurate short read alignment with Burrows-Wheeler transform. Bioinformatics 25:1754-1760.

MacDonald JR, Ziman R, Yuen RKC, Feuk L, Scherer SW. (2014) The Database of Genomic Variants: a curated collection of structural variation in the human genome. Nucleic Acids Res 42:D986-992.

McGowan-Jordan J, Simons A, Schmid M. (2016) ISCN 2016: An International system for human cytogenomic nomenclature. Karger, Basel, New York. 
McKusick VA. (1998) Mendelian inheritance in Man. A catalog of human genes and genetic disorders. 12th edn. Johns Hopkins University Press, Baltimore, MD.

Miller DT, Adam MP, Aradhya S, Biesecker LG, Brothman AR, Carter NP, Church DM, Crolla JA, Eichler EE, Epstein CJ, Faucett WA, Feuk L, Friedman JM, Hamosh A, Jackson L, Kaminsky EB, Kok K, Krantz ID, Kuhn RM, Lee C, Ostell JM, Rosenberg C, Scherer SW, Spinner NB, Stavropoulos DJ, Tepperberg JH, Thorland EC, Vermeesch JR, Waggoner DJ, Watson MS, Martin CL, Ledbetter $\mathrm{DH}$. (2010) Consensus statement: chromosomal microarray is a first-tier clinical diagnostic test for individuals with developmental disabilities or congenital anomalies. Am J Hum Genet 86:749-764.

Moore BL, Aitken S, Semple CA. (2015) Integrative modeling reveals the principles of multiscale chromatin boundary formation in human nuclear organization. Genome Biol 16,110.

Novara F, Rinaldi B, Sisodiya SM, Coppola A, Giglio S, Stanzial F, Benedicenti F, Donaldson A, Andrieux J, Stapleton R, Weber A, Reho P, van Ravenswaaij-Arts C, Kerstjens-Frederikse WS, Vermeesch JR, Devriendt K, Bacino CA, Delahaye A, Maas SM, Iolascon A, Zuffardi O. (2017) Haploinsufficiency for ANKRD11flanking genes makes the difference between KBG and 16q24.3 microdeletion syndromes: 12 new cases. Eur J Hum Genet 25:694-701.

Ordulu Z, Wong KE, Currall BB, Ivanov AR, Pereira S, Althari S, Gusella JF, Talkowski ME, Morton CC. (2014) Describing sequencing results of structural chromosome rearrangements with a suggested next-generation cytogenetic nomenclature. $\mathrm{Am}$ J Hum Genet 94:695-709.

Rao SSP, Huntley MH, Durand NC, Stamenova EK, Bochkov ID, Robinson JT, Sanborn AL, Machol I, Omer AD, Lander ES, Aiden EL. (2014) A 3D map of the human genome at kilobase resolution reveals principles of chromatin looping. Cell 159:1665-1680.

Riggs ER, Andersen EF, Cherry AM, Kantarci S, Kearney H, Patel A, Raca G, Ritter DI, South ST, Thorland EC, Pineda-Alvarez D, Aradhya S, Martin CL. (2019) Technical standards for the interpretation and reporting of constitutional copynumber variants: a joint consensus recommendation of the American College of Medical Genetics and Genomics (ACMG) and the Clinical Genome Resource (ClinGen). Genet Med 1-13.

Quinlan AR, Hall IM. (2010) BEDTools: a flexible suite of utilities for comparing genomic features. Bioinformatics 26:841-842.

Sánchez-Ferrero E, Coto E, Beetz C, Gámez J, Corao Al, Díaz M, Esteban J, del Castillo E, Moris G, Infante J, Menéndez M, Pascual-Pascual SI, López de Munaín A, Garcia-Barcina MJ, Alvarez V. (2013) SPG7 mutational screening in spastic paraplegia patients supports a dominant effect for some mutations and a pathogenic role for p.A510V. Clin Genet 83:257-262.

Skraban CM, Wells CF, Markose P, Cho MT, Nesbitt AI, Au PYB, Begtrup A, Bernat JA, Bird LM, Cao K, de Brouwer APM, Denenberg EH, Douglas G, Gibson KM, Grand K, Goldenberg A, Innes AM, Juusola J, Kempers M, Kinning E, Markie DM, Owens MM, Payne K, Person R, Pfundt R, Stocco A, Turner CLS, Verbeek NE, Walsh LE, Warner TC, Wheeler PG, Wieczorek D, Wilkens AB, ZonneveldHuijssoon E; Deciphering Developmental Disorders Study, Kleefstra T, Robertson SP, Santani A, van Gassen KLI, Deardorff MA. (2017) WDR26 haploinsufficiency causes a recognizable syndrome of intellectual disability, seizures, abnormal gait, and distinctive facial features. Am J Hum Genet 101:139-148. 
Stelzer G, Rosen N, Plaschkes I, Zimmerman S, Twik M, Fishilevich S, Stein TI, Nudel R, Lieder I, Mazor Y, Kaplan S, Dahary D, Warshawsky D, Guan-Golan Y, Kohn A, Rappaport N, Safran M, Lancet D. (2016) The GeneCards suite: from gene data mining to disease genome sequence analyses. Curr Protoc Bioinformatics 54:1.30.1-1.30.33.

Talkowski ME, Ernst C, Heilbut A, Chiang C, Hanscom C, Lindgren A, Kirby A, Liu S, Muddukrishna B, Ohsumi TK, Shen Y, Borowsky M, Daly MJ, Morton CC, Gusella JF. (2011) Next-generation sequencing strategies enable routine detection of balanced chromosome rearrangements for clinical diagnostics and genetic research. Am J Hum Genet 88:469-481.

Tarasov A, Vilella AJ, Cuppen E, Nijman IJ, Prins P. (2015) Sambamba: fast processing of NGS alignment formats. Bioinformatics 31:2032-2034.

Wright CF, Fitzgerald TW, Jones WD, Clayton S, McRae JF, Kogelenberg M, King DA, Ambridge K, Barret DM, Bayzetinova T, Bevan AP, Bragin E, Chatzimichali EA, Gribble S, Jones P, Krishnappa N, Mason LE, Miller R, Morley KI, Parthiban V, Prigmore E, Rajan D, Sifrim A, Swaminathan J, Tivey AR, Middleton A, Parker M, Carter NP, Barrett JC, Hurles ME, FitzPatrickDR, Firth HV. (2015) Genetic diagnosis of developmental disorders in the DDD study: a scalable analysis of genome-wide research data. Lancet 385:1305-1314.

Zerbino DR, Achuthan P, Akanni W, Amode MR, Barrell D, Bhai J, Billis K, Cummins C, Gall A, Girón CG, Gil L, Gordon L, Haggerty L, Haskell E, Hourlier T, Izuogu OG, Janacek SH, Juettemann T, To JK, Laird MR, Lavidas I, Liu Z, Loveland JE, Maurel T, McLaren W, Moore B, Mudge J, Murphy DN, Newman V, Nuhn M, Ogeh D, Ong CK, Parker A, Patricio M, Riat HS, Schuilenburg H, Sheppard D, Sparrow H, Taylor K, Thormann A, Vullo A, Walts B, Zadissa A, Frankish A, Hunt SE, Kostadima M, Langridge N, Martin FJ, Muffato M, Perry E, Ruffier M, Staines DM, Trevanion SJ, Aken BL, Cunningham F, Yates A, Flicek P. (2018) Ensembl 2018. Nucleic Acids Res 46:D754-D761. 